\title{
DigITALCOMMONS
}

@WAYNESTATE-

Wayne State University

Wayne State University Dissertations

$1-1-2016$

\section{Arbitrary Lagrangian-Eulerian Method Investigation On Fuel Tank Strap Simulation Under Proving Ground Condition}

Guangtian Song

Wayne State University,

Follow this and additional works at: https://digitalcommons.wayne.edu/oa_dissertations

Part of the Other Mechanical Engineering Commons

\section{Recommended Citation}

Song, Guangtian, "Arbitrary Lagrangian-Eulerian Method Investigation On Fuel Tank Strap Simulation Under Proving Ground Condition" (2016). Wayne State University Dissertations. 1593.

https://digitalcommons.wayne.edu/oa_dissertations/1593 


\title{
ARBITRARY LAGRANGIAN-EULERIAN METHOD INVESTIGATION ON FUEL TANK STRAP SIMULATION UNDER PROVING GROUND CONDITION
}

\author{
by \\ GUANGTIAN SONG \\ DISSERTATION \\ Submitted to the Graduate School \\ of Wayne State University, \\ Detroit, Michigan \\ in partial fulfillment of the requirements \\ for the degree of
}

DOCTOR OF PHILOSOPHY

2016

MAJOR: MECHANICAL ENGINEERING

Approved By:

Advisor

Date 
(C) COPYRIGHT BY

GUANGTIAN SONG

2016

All Rights Reserved 


\section{DEDICATION}

I dedicate this dissertation work to my lovely family, my parents, passing father during my

PH. D. study-Lizhu Song and dear mother-Cuifen Chen, my wife, Xuerong Lu, and my son, Isaac Song. 


\section{ACKNOWLEDGEMENTS}

First and foremost, I would like to thank my advisor, Dr. Chin-An Tan. I would not finish this dissertation without his support and encouragement through all the academic courses and research. His wisdom, knowledge, commitment and demand to the highest standards keeps on inspiring and motivating me. I enjoy very much this wonderful eight years' science journey he guides me, which will greatly benefit my career and life. I also wish to thank my committee members, Dr. Golam M. Newaz, Dr. Xin Wu, and Dr. Christopher Eamon, who are more than generous with their expertise, precious time, and valuable guidance to review my research proposal many times. I need thank all my Ford colleagues, Dr. Yongquan Liu, Dr.Xianqiang Lu, Dr. Veeraraghavan Arun, Dr. Kun-Tien Shu, Dr. Bijan Khatib-Shahidi, Mr. Amar Ourchane, technical support from Radioss Corporation, Dr. Jean-Pierre Bobineau and Mickael Pourrat, and technical support from nCode International, Mr. Jeffrey Mentley, to discuss the related topics. Appreciation is necessary to Livermore Software Technology Corporation to permit the reprint of LS-DYNA Theory Manual. Last but not least, I deeply acknowledge the love, encouragement and support for these many years from my parents, my wife, and my son, especially my wife takes care of most family life. 


\section{TABLE OF CONTENTS}

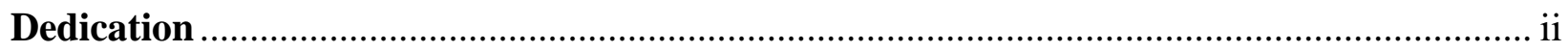

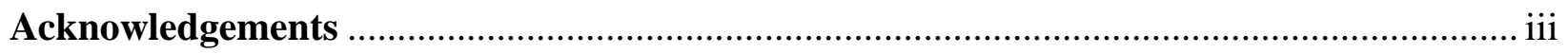

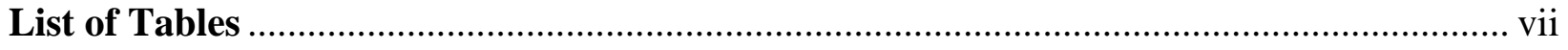

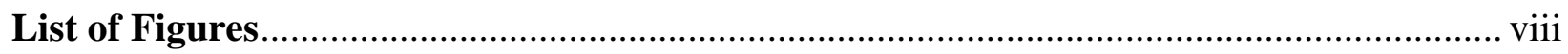

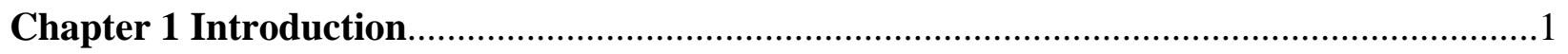

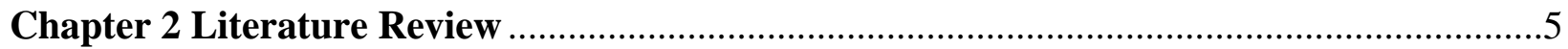

2.1 The Simulation of Sloshing and Ballooning in the Fuel Tank for High Speed Impact.7

2.2 Fuel Tank Strap Fatigue Sensitivity Study under Fuel Level Variation and Payload Variation ...........................................................................................................

2.3 CAE Fatigue Prediction of Fuel Tank Straps using Proving Ground Loads ................12

2.4 Literature Review Summary ………………………..............................................13

2.4.1 Load Cell...............................................................................................14

2.4.2 Virtual Fluid Mass Method............................................................................16

2.4.3 Modal Transient Response........................................................................17

2.4.4 Modal versus Direct Transient Response ....................................................20

Chapter 3 Governing Equation of Arbitrary Lagrangian-Eulerian .....................................22

3.1 ALE Form of Conservation Equations ………………….......................................22

3.2 Arbitrary Lagrangian-Eulerian Update Procedure........................................................24

3.2.1 Overall Flow of ALE Steps........................................................................24

3.2.2 Mesh Smoothing Algorithm ......................................................................24

3.2.3 Advection Algorithm .........................................................................24

3.2.2 The Manual Rezone ................................................................................25 


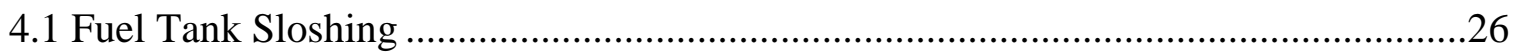

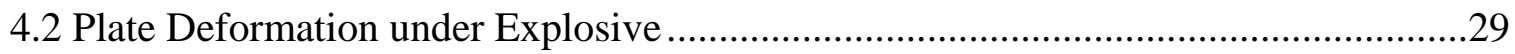

4.3 High Strength Steel Plate Crack Initiation and Propagation under Explosive..............34

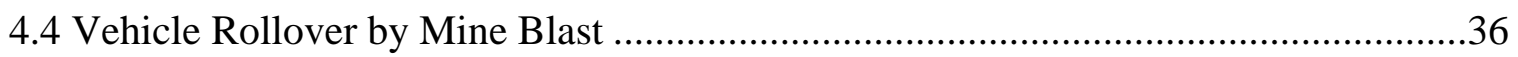

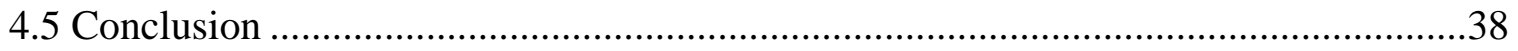

\section{Chapter 5 Arbitrary Lagrangian-Eulerian Method in Fuel Tank Strap Simulation under Proving Ground Condition ...............................................39}

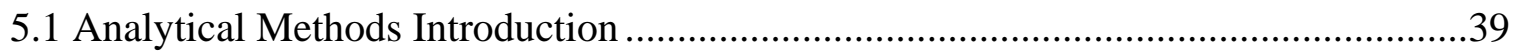

5.1.1 Common Mass Nonlinear Method............................................................40

5.1.2 Arbitrary Lagrange-Euler (ALE) Method.................................................40

5.1.3 Smooth Particles Hydrodynamics (S.P.H.) Method ..................................41

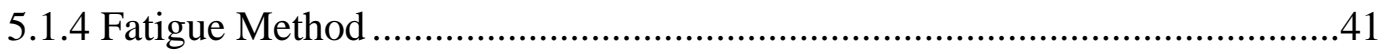

5.1.5 Equation of Motion Explicit Time Integration ..........................................42

5.1.6 Signal Processing in Matlab.................................................................44

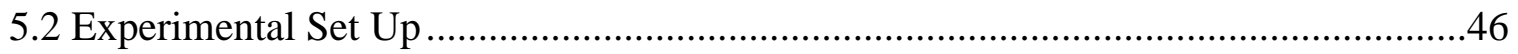

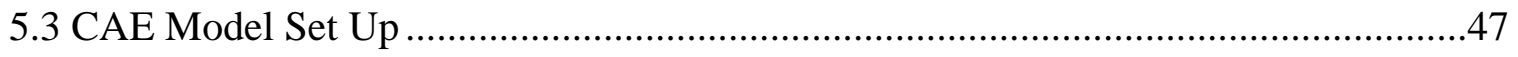

5.3.1 Material property for fuel tank strap and fuel tank ................................47

5.3.2 Modeling of Joints between Hydraulic Actuator and Frame .......................49

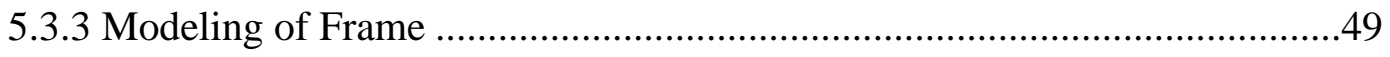

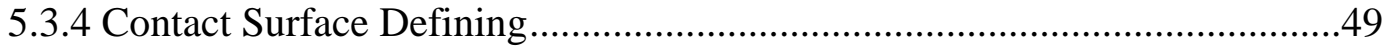

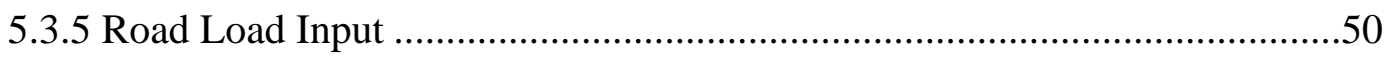

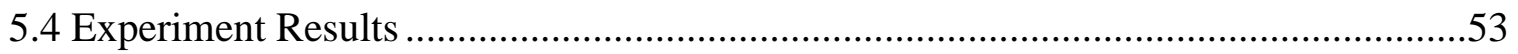

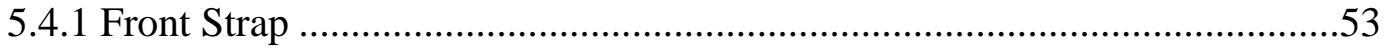


5.4.2 Rear Strap.

5.5 CAE and Experiment Results Comparison..........................................................54

5.5.1 Stress Analysis Result Comparison for Rear Strap........................................54

5.5.2 Stress Analysis Result Comparison between Front and Rear Strap ..............57

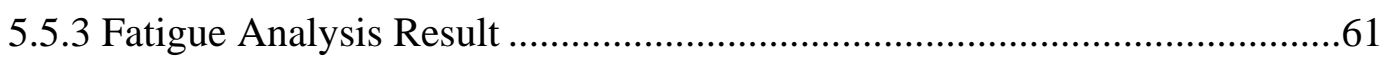

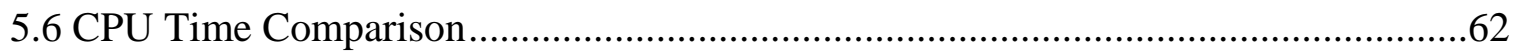

5.7 Conclusion .........................................................................................................62

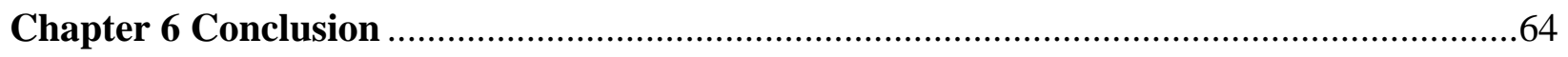

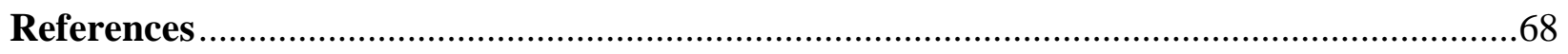

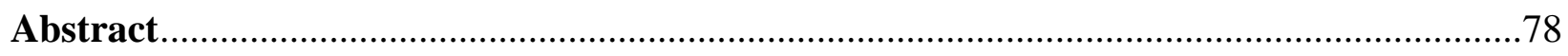

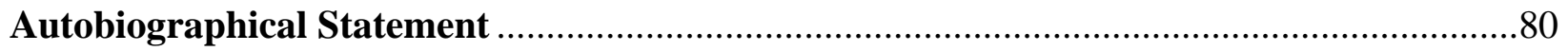




\section{LIST OF TABLES}

Table 2-1: Modal versus Direct Transient Response ..............................................................20

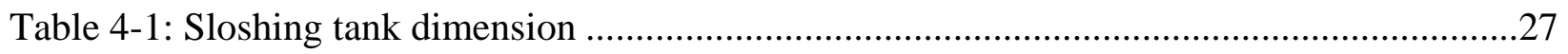

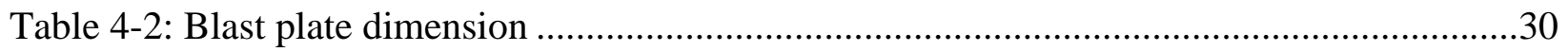

Table 4-3: Blast plate material properties .........................................................................30

Table 5-1: Material property for fuel tank strap and fuel tank ...........................................47

Table 5-2: Proving Ground Route Event ...........................................................................50

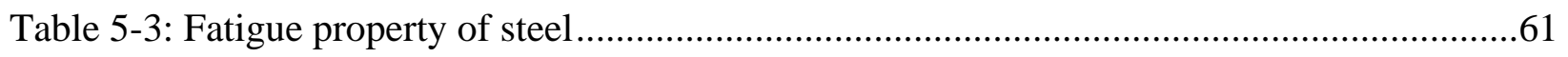

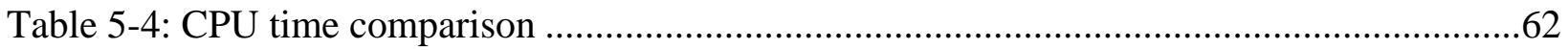




\section{LIST OF FIGURES}

Figure 2-1: Fuel sloshing in 20\%, 50\%, and 95\%-full tank.................................................

Figure 2-2: Surface stress contour of 20\%, 50\%, and 95\%-full tank ......................................9

Figure 2-3: Strap connections to cross member................................................................10

Figure 2-4: CAE correlation of front strap fatigue ..............................................................11

Figure 2-5: CAE correlation of rear strap fatigue .............................................................11

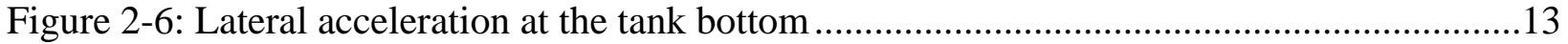

Figure 2-7: Sample \#2, the rear strap cracked due to a loose strap and CAE fatigue prediction ..13

Figure 3-1: Lagrangian mesh, Eulerian mesh, and ALE mesh comparison ..............................22

Figure 3-2: Grid velocity and material velocity in compressible Naiver Stokes equation ............23

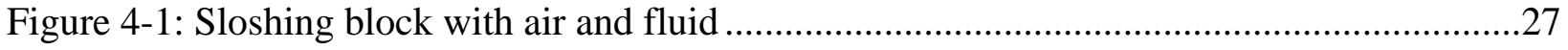

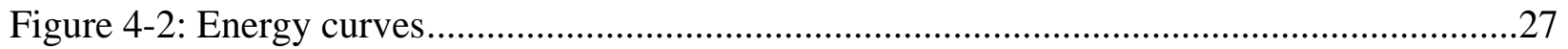

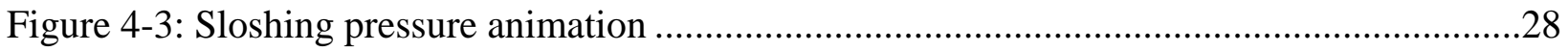

Figure 4-4: Sloshing pressure iso-surface animation........................................................29

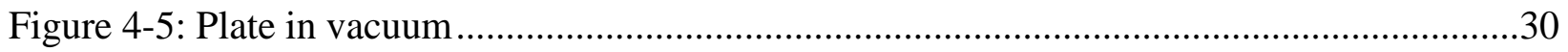

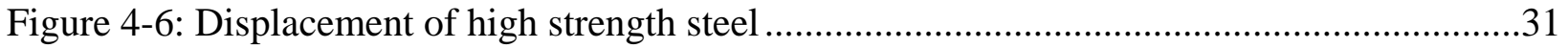

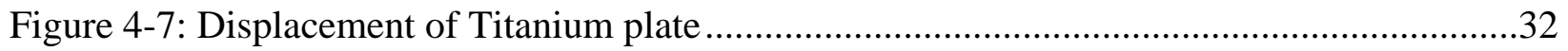

Figure 4-8: Displacement of enter point of two plates ......................................................32

Figure 4-9: Effective Plastic Strain of center point of two plates.............................................33

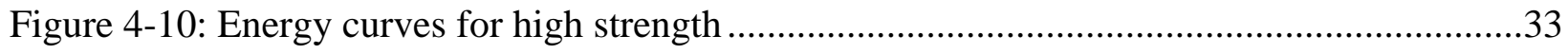

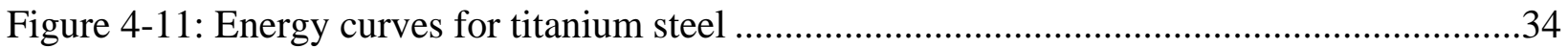

Figure 4-12: Crack Initiation in high strength steel plate ......................................................35

Figure 4-13: Energy curves for high strength steel plates in Crack........................................36 
Figure 4-14: Vehicle in vacuum with explosive under tire.

Figure 4-15: Vehicle rollover by explosive .37

Figure 4-16: Energy curves in explosive .38

Figure 5-1: Explicit time integration. .43

Figure 5-2: Fuel tank strap study setup in test .46

Figure 5-3(a): CAE setup in Nonlinear Common Mass Method .47

Figure 5-3(b): CAE setup in Nonlinear ALE Method .47

Figure 5-4: Fuel tank strap plastic stress strain curve. .48

Figure 5-5: Fuel tank plastic stress strain curve. .48

Figure 5-6: Joint between hydraulic actuator and frame in test .49

Figure 5-7: Acceleration load input in testing .... .52

Figure 5-8: Filtered seven channel road load displacement input ...... .53

Figure 5-9(a): Fuel sloshing in the fuel tank at 0 Seconds .55

Figure 5-9(b): Fuel sloshing in the fuel tank at 3 Seconds .55

Figure 5-9(c): Fuel sloshing in the fuel tank at 6 Seconds .55

Figure 5-9(d): Fuel sloshing in the fuel tank at 9 Seconds .55

Figure 5-9(e): Fuel sloshing in the fuel tank at 12 Seconds .55

Figure 5-10: Cracks in outboard leading edge of rear strap..... .56

Figure 5-11: Von Mises stress in rear strap with Linear Fluid Volume Properties Definition method. .56

Figure 5-12: Von Mises stress In Rear Strap in Common Mass Nonlinear method.... . .56

Figure 5-13: Von Mises stress in Rear Strap in ALE Nonlinear method .56

Figure 5-14: Plastic strain contour in rear strap in Common Mass Nonlinear method .57

Figure 5-15: Plastic strain contour in rear strap in ALE Nonlinear method. .57 
Figure 5-16: Von Mises stress contour in Left-to-Right side view in Linear Fluid Volume Properties Definition method

Figure 5-17: Von Mises stress contour in Left-to-Right side view in Common Mass

Nonlinear method.

Figure 5-18: Von Mises stress contour in Left-to-Right side view in ALE Nonlinear method.....58

Figure 5-19 (a): Plastics strain contour at 1 second in Common Mass Nonlinear method ...........60

Figure 5-19 (b): Plastics strain contour at 3 seconds in Common Mass Nonlinear method..........60

Figure 5-19 (c): Plastics strain contour at 9 seconds in Common Mass Nonlinear method..........60

Figure 5-19 (d): Plastics strain contour at 12 seconds in Common Mass Nonlinear method........60

Figure 5-20 (a): Plastics strain contour at 1 second in Common Mass Nonlinear method ...........60

Figure 5-20 (b): Plastics strain contour at 3 seconds in Common Mass Nonlinear method..........60

Figure 5-20 (c): Plastics strain contour at 9 seconds in Common Mass Nonlinear method..........60

Figure 5-20 (d): Plastics strain contour at 12 seconds in Common Mass Nonlinear method........60

Figure 5-21: Fatigue life contour in Left-to-Right side view in Common Mass Nonlinear method.

Figure 5-22: Fatigue life contour in Left-to-Right side view in ALE Nonlinear method..............62 


\section{CHAPTER 1 INTRODUCTION}

The Arbitrary Lagrangian-Eulerian (ALE) is a hybrid finite element formulation that can alleviate many of the drawbacks from the traditional Lagrangian-based and Eulerian-based finite element simulations. Lagrangian-based finite element formulations is that the computational system moves with the material and main drawback is that it will face severe problems to deal with strong distortions in the computational domain. Eulerian-based finite element formulations is that the computational system is a prior fixed in space and the main disadvantages are: 1) material interfaces lose their sharp definitions as the fluid moves through the mesh and undergo large distortions, which often leads to the inaccuracies in basic Eulerian calculations, 2) local regions of fine resolution are difficult to achieve.

The use of Arbitrary Lagrangian-Eulerian (ALE) computer codes has been an enabling technology for many important applications. These computer codes are developed through combining modern algorithms for Lagrangian hydrodynamics, meshing technology and remap methods developed for high-resolution Eulerian methods.

When using the ALE technique in engineering simulations, the computational mesh inside the domains can move arbitrarily to optimize the shapes of elements, while the mesh on the boundaries and interfaces of the domains can move along with materials to precisely track the boundaries and interfaces of a multi-material system.

ALE-based finite element formulations can reduce to either Lagrangian-based finite element formulations by equating mesh motion to material motion or Eulerian-based finite element formulations by fixing mesh in space. Therefore, one finite element code can be used to perform comprehensive engineering simulations, including heat transfer, fluid flow, fluid-structure interactions and metal-manufacturing. 
In automotive CAE durability analysis, simulation of dynamic stress and fatigue life of fuel tank straps is a complex problem. Typically a fuel tank is held with fuel tank straps. Its movement lies in the domain of nonlinear large rotation dynamics. Moreover, the sloshing behavior in the fuel tank makes the problem even more intricate.

Nowadays, CAE methods for fuel tank strap simulation under proving ground condition are limited to 1 . Concentrated Mass Method-a mass element located in center of gravity of fuel with equivalent fuel mass and connected to the fuel tank through weighted motion constraints, 2 . Common Mass Method-adjusting tank density to account for the mass of tank and fuel together, 3. Fluid Volume Properties Definition Method-applying MFUILD card in NASTRAN to define a fuel material property to generate a virtual mass for the element envelope that contains the volume of the incompressible fuel. However, above methods still can't predict cracks initiation and sequence precisely as the testing, because those methods ignored the critical fuel sloshing effects which causes the fuel mass redistribution along with the vehicle driving.

Therefore, motivation is originated to investigate how important and how much the fuel sloshing during vehicle driving will impact the fuel tank and fuel tank strap stress and fatigue life. Through comparing different available CAE methods mentioned above, the objective of this research is to measure how accurate Arbitrary Lagrangian-Eulerian method to simulate fuel sloshing will be for the fuel tank and fuel tank strap movement under proving ground conditions. Altair RADIOSS, a multidisciplinary finite element solver developed by Altair Engineering, is used for this highly non-linear transient dynamics system.

In the end of this research, the stress distribution of the fuel tank strap can be well predicted with Arbitrary Lagrangian-Eulerian Method (ALE) to simulate fuel and fuel vapor. The world reputational finite element based fatigue code, nCode DesignLife, is used to predict the fatigue life 
of the fuel tank straps from stress and strain time history result. Overall, the analyses have accurately predicted the crack initiation sequence and locations in the fuel tank straps, and show good correlation with test. The utilization of this method will give design right direction to minimize the iteration of lab testing and expedite the design period.

The contents of this Ph.D. thesis are organized into the following chapters:

- Chapter 1: Introduction

This Chapter introduces the research motivation, objective, and the successful application of Arbitrary Lagrange-Euler Method (ALE) in fuel sloshing simulation to predict the cracks initiation location and sequence and fatigue life of the fuel tank straps under proving ground condition.

- Chapter 2: Literature Review

This chapter gives a general literature review about the development history and current application areas of Arbitrary Lagrangian-Eulerian Method (ALE), such as Forming Processes, Two-Phase Flows with Phase Change and Heat Transfer of Computer Chips, Plasma Physics. Further literature review is the current CAE methods application in auto industry fuel tank and fuel tank strap simulation and those methods' weakness are emphasized as well.

- Chapter 3: Governing Equations of Arbitrary Lagrangian-Eulerian Method

This chapter introduces all the equations and smoothing and advection algorithm for Arbitrary Lagrangian-Eulerian equation that most are from publication by Livermore Software Technology Corporation (LSTC).

- Chapter 4: Arbitrary Lagrangian-Eulerian Application Examples 
This chapter gives four simple examples to show how Arbitrary LagrangianEulerian Method (ALE) method can be applied in fuel sloshing simulation, explosive simulation for plate crack initiation and propagation and military ground vehicle rollover.

- Chapter 5: Arbitrary Lagrangian-Eulerian Method in Fuel Tank Strap Simulation under Proving Ground Condition

This chapter describes the detail in five areas how ALE method is applied in fuel tank strap simulation under proving ground condition.

1. Apply ALE method to simulate fuel sloshing and integrate with proving ground time history load.

2. Set up procedure how to simplify CAE model, coarse the model and replace some contact surface with common nodes method, to reduce CPU time.

3. Simplify and process the testing load in MATLAB to reduce CPU time and avoid the numerical issue in explicit dynamic transient code.

4. Define nonlinear material properties in the model to trace plastic deformation.

5. Interface with fatigue code through programing to post process the intermediate results files.

- Chapter 6: Conclusion

This chapter concludes the total four major achievement for the research and recommends the future work. 


\section{CHAPTER 2 LITERATURE REVIEW}

ALE methods were introduced in 1974 by Hirt, Amsden, and Cook [1], but needed the development and wide-spread use of high-resolution method such as FCT [2], or slope-limiters [3] to become accurate enough for practical application. This was due to the overly dissipative remap associated with the use of upwind or donor-cell differencing. The upwind-type of differencing was necessary to produce physically bounded quantities in the remap essential for challenging problems.

In addition, ALE methods have benefited from the development of modern Lagrangian methods in the past 15 years. Two primary thrusts have shaped Lagrangian hydrodynamics during this period, one is the form of the discrete difference equations, and the second is the form of the artificial viscosity used to compute shocked solutions. The discrete difference equations have been improved radically through the use of mimetic principles, symmetry and conservation [4-9]. The second development has utilized the technology used to develop high-resolution methods to limit the amount of dissipation in calculations [5][10][11]. These methods combined to improve the quality of Lagrangian integrators substantially over the classical methods [12-14].

Finally, the meshing methods have improved, albeit to a lesser extent than the integration method. Thus, meshing methods remain an active area of research with the promise of determining progress in achieving higher quality with ALE simulations. The latest ALE algorithms are continually developed by, such as, ALEGRA [15], LS-DYNA, RADIOSS, etc.

With the development of ALE method, ALE method is getting more and more application in many area that involve fluid structure interaction. Christiaan Stoker performed simulation of forming process in ALE method and two academic test cases [16]. It is concluded that the remap of state variables is carried out sufficiently accurately, the meshing step is crucial for the 
effectiveness of the ALE method, and the ALE method in combination with remeshing should be used. Gustavo Rabello Dos Anjos proposed ALE method to study two-phase flow and heat transfer for interlayer cooling of the new generation of multi-stacked computer chips [17]. The fluid flow equations are developed in 3-dimensions based on the Arbitrary Lagrangian-Eulerian formulation (ALE) and the Finite Element Method (FEM), creating a new two-phase method with an improved model for the liquid-gas interface. A new adaptive mesh update procedure is also proposed for effective management of the mesh at the two-phase interface to remove, add and repair surface elements, since the computational mesh nodes move according to the flow. Static and dynamic tests have been carried out to validate the method to simulate two-phase flows which provides good accuracy to describe the interfacial forces and bubble dynamics and is being considered for two-phase interlayer cooling in 3D-IC computer chips. Milan Kucha r'r'k developed ALE code in Plasma Physics [18]. Three sets of laser plasma simulations inspired by the real experiments are performed - the interaction of a laser beam with a massive target, ablative acceleration of small Aluminum disc flyer irradiated by a laser beam, and the high velocity impact of such accelerated disc onto a massive Aluminum target. The standard Lagrangian simulation in high velocity impact problem fails, and the complete ALE methodology in simulations of all types of problems show reasonable agreement with the experimental results.

In automotive CAE analysis, simulation of fuel tank potential leakage under impact in crashworthiness and dynamic stress and fatigue life of fuel tank straps under proving ground condition in durability are active problems for vehicle design. Typically a fuel tank is held with fuel tank straps. Its movement lies in the domain of nonlinear large rotation dynamics. Moreover, the sloshing behavior in the fuel tank makes the problem even more intricate. 


\subsection{The Simulation of Sloshing and Ballooning in the Fuel Tank for High Speed Impact}

Tang, B., Guha, S., Tyan, T., Doong, J. et al., described the simulation of sloshing and ballooning in fuel tanks for high speed impacts [20]. During high-speed impact on the other hand, there is significant bulging of the fuel tank if it is nearly full, while vortices and cavities may form with partial filling. In these cases, the internal fuel and vapor pressure distribution can change; thus, affecting the distribution of stress on the tank. The objective is to study these phenomena using the currently available Arbitrary Lagrangian-Eulerian methodology and thus improve fuel tank design by a direct application of CAE.

To help understand the responses of the fuel, vapor and tank under high speed impact condition, three fuel levels, $20 \%, 50 \%$ and $95 \%$, are the three cases induced in the study, but the conclusion is obvious. The $95 \%$ filled level produces much larger tank deformation, higher stress level, higher pressure and wider pressure distribution than the other two lower fuel level tanks do. Both $20 \%$ and $50 \%$ levels are not much different from each other in all aspects. Their difference from an undeformed tank is not very substantial as well. 


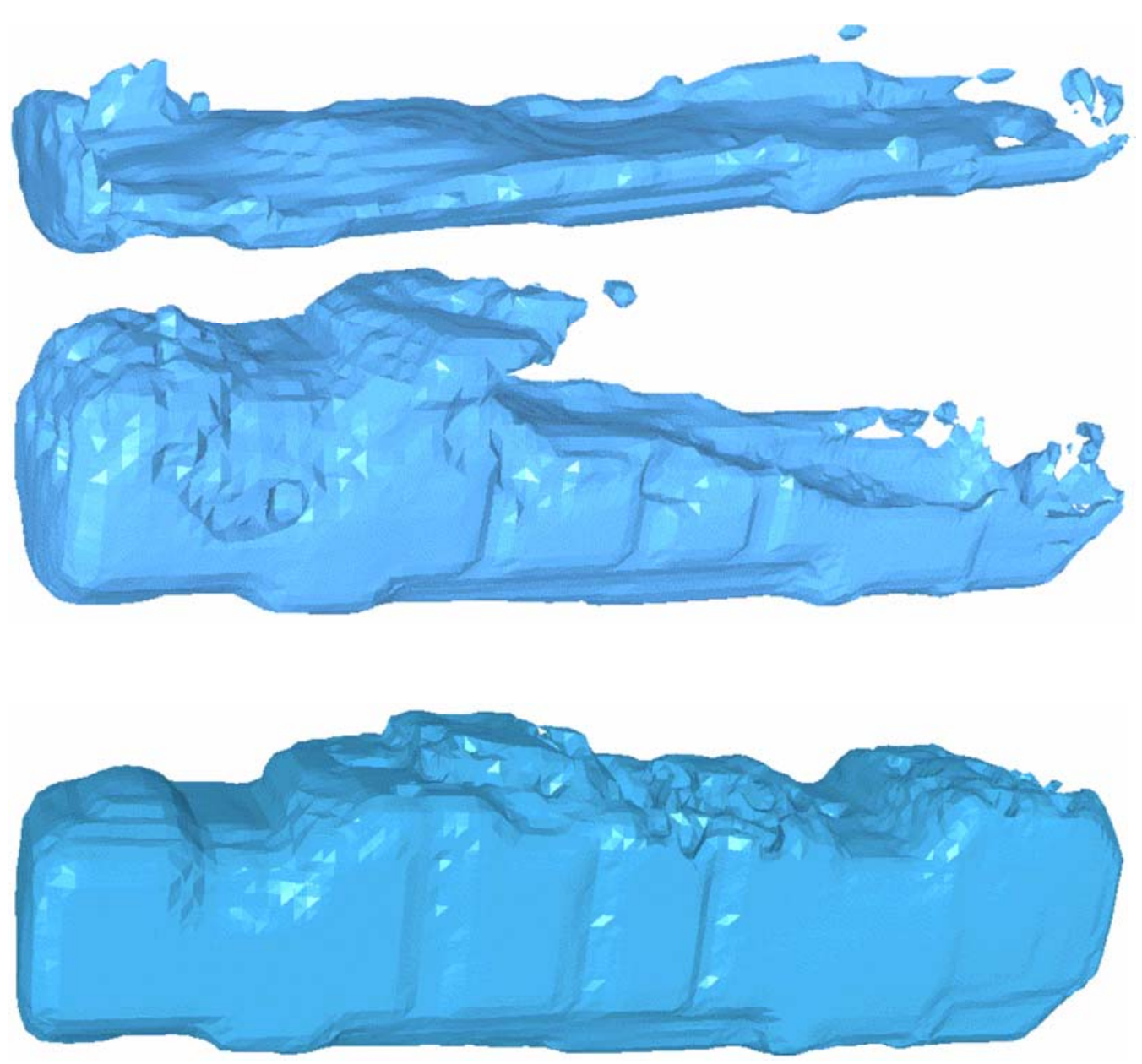

Figure 2-1: Fuel sloshing in 20\%, 50\%, and 95\%-full tank [20] 

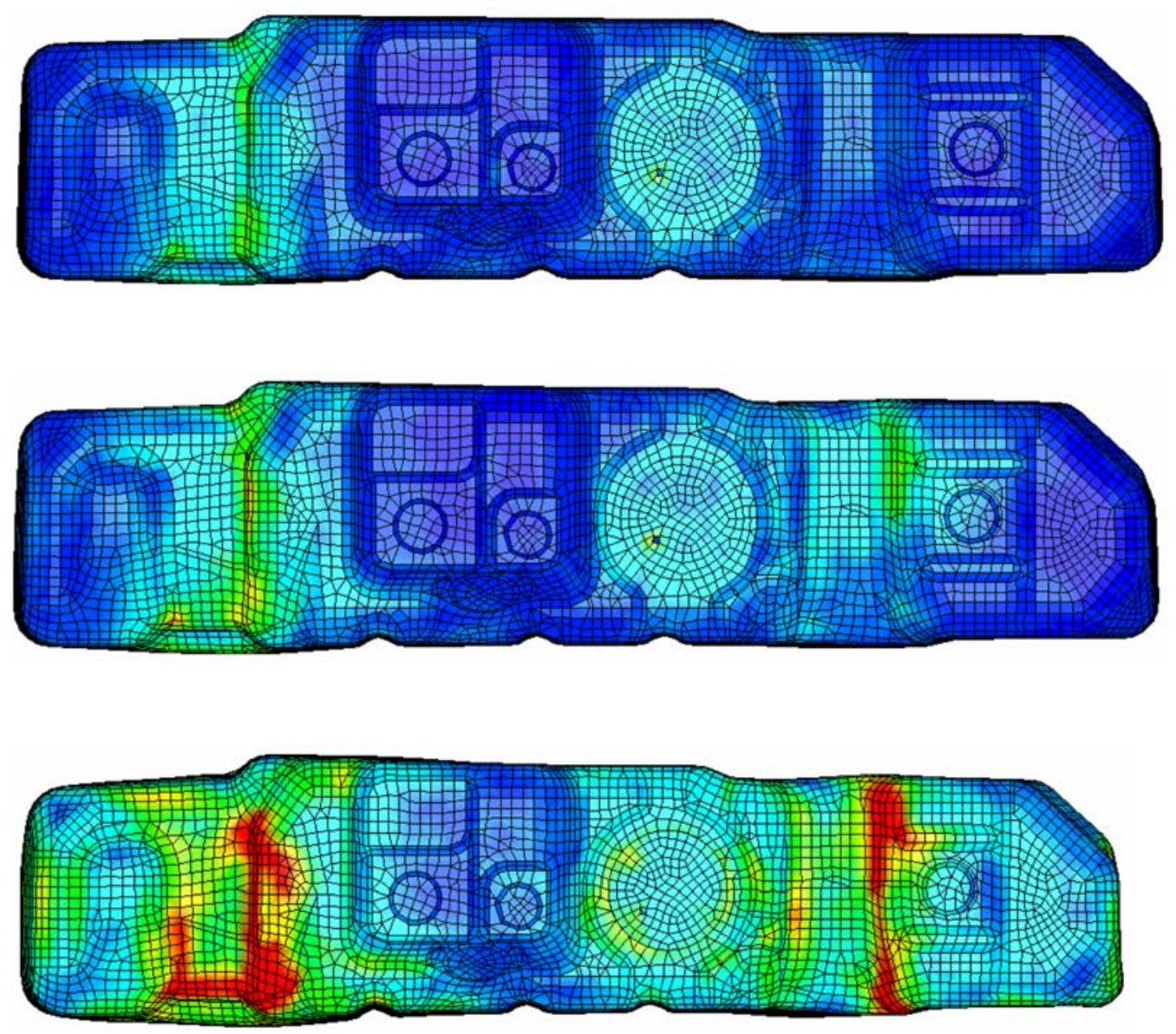

Figure 2-2: Surface stress contour of $20 \%, 50 \%$, and 95\%-full tank [20]

\subsection{Fuel Tank Strap Fatigue Sensitivity Study under Fuel Level Variation and Payload Variation}

Lin, B., et al, published the research on fuel tank strap fatigue sensitivity study under fuel level variation and payload variation [21]. Proving ground load data acquisition was made at fuel tank strap attachment locations to cross members. Load cells were used for I/B (inboard) connections. Strain gages were used for O/B (outboard) T-slot connections. Load data (Fx, Fy and Fz) was collected on dozens of different road segments for each of the different fuel level conditions $(1 / 4,1 / 2,3 / 4$ and full) in fuel tank. 


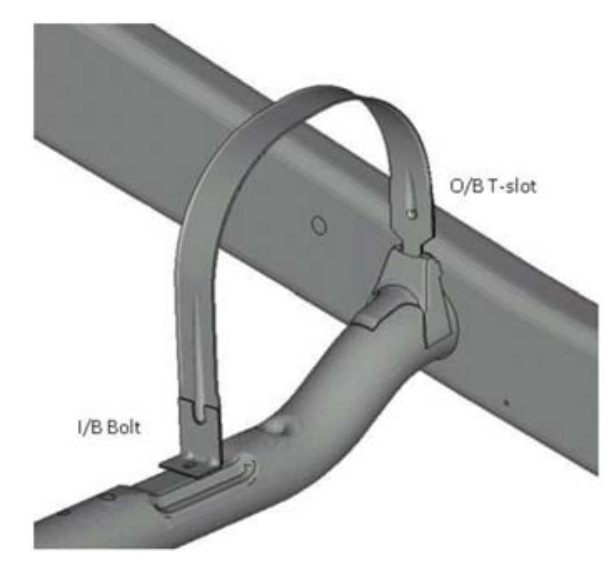

Figure 2-3 Strap connections to cross member [21]

In this study, strap load was directly acquired and could be used directly. As a result, a simple quasi-static stress analysis could be used in stress calculations since all nonlinear contact behavior and fuel sloshing interaction with tank as well as inertia force of fuel were already included in acquired load data.

In $\mathrm{CAE}$, the front strap of the fuel tank is connected to a cross member through a rigid element representing bolt at inboard side. Contact is defined between the strap foot (slave surface) and cross member (master surface) in contact area. The front portion of fuel tank is modeled. Contact between strap (slave surface) and fuel tank (master surface) is defined. Load is applied to the outboard end in $\mathrm{x}, \mathrm{y}$ and $\mathrm{z}$ directions. In CAE simulation after three unit-load cases are analyzed in ABAQUS for stress output, stress time histories are built through superposition technique inside fatigue software nCode Design-life. Then fatigue life is calculated through nCode Design-life using rain flow counting technique and strap steel E-N curve. 

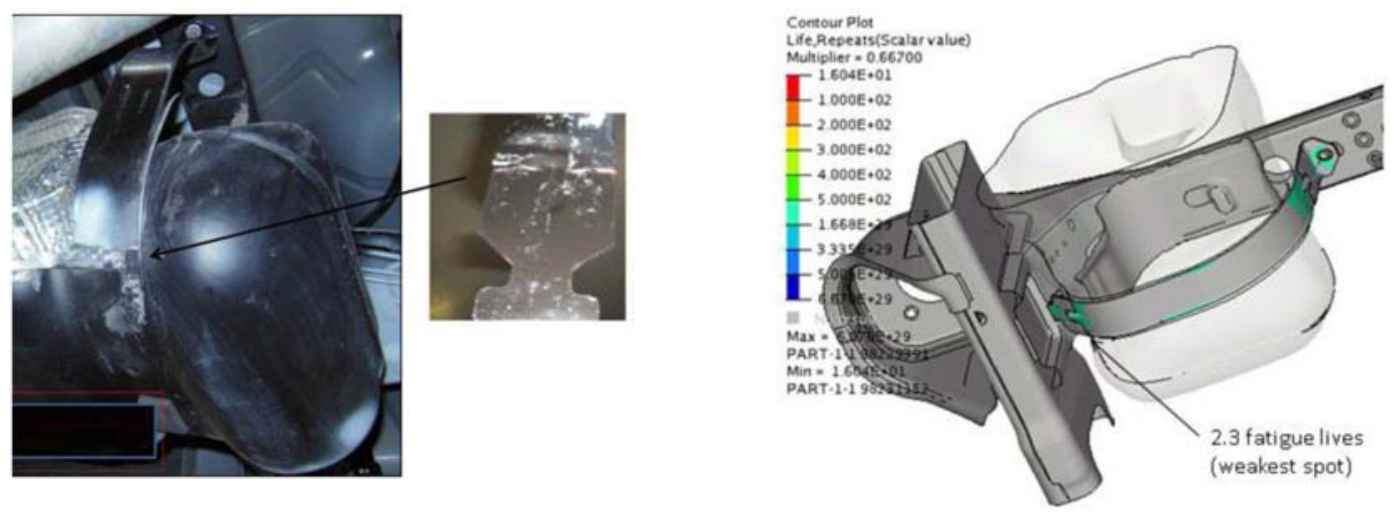

Figure 2-4 CAE correlation of front strap fatigue [21]

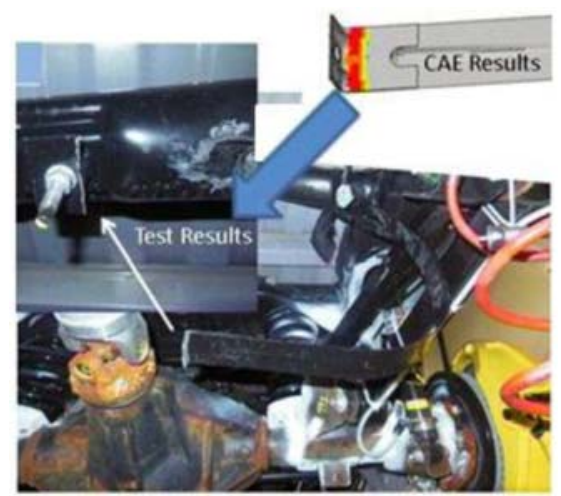

Figure 2-5 CAE correlation of rear strap fatigue [21]

From CAE fatigue results, it is observed that every quarter tank difference of fuel will change the rear fuel tank fatigue life by one order of magnitude, because the force on the rear strap will increase with the weight of fuel tank increasing.

In order to have better understanding of fuel tank strap durability load and performance, strap load data acquisition was also conducted at different payload condition. In this study only the full fuel level was studied. One may notice that higher payload is helping fuel tank strap durability performance, because the higher load will reduce the rebound amplitude of vehicle rear. 


\subsection{CAE Fatigue Prediction of Fuel Tank Straps using Proving Ground Loads}

Qin, P. and D'Souza, S. published their research in CAE fatigue prediction of fuel tank straps using proving ground loads [22].

At the proving grounds (PG), acceleration data on the tank and strain data on the straps can be acquired, then used to back calculate hydraulic load inputs to the MAST based on transfer functions, applied to the FEA model of the fuel tank system through the body structure, and strain data for correlation.

To represent the fuel and the fuel-to-tank interaction more accurately than the lumped mass method, the MFLUID method (also called the virtual fluid mass method) was applied [23]. The MFLUID method generates a mass matrix of the wetted grids on the tank to represent the fluid and fluid to-structure interaction. This method assumes that the fluid is incompressible and the free surface has zero pressure with no sloshing effects. Also, no viscous (rotational flow) effects exist.

CAE Modal transient response method is applied to get modal strain for the straps and modal coordinate time history, then CAE modal fatigue analysis is followed to get fatigue result for strap. Correlation is shown in Figure 2-6 and 2-7 respectively. 


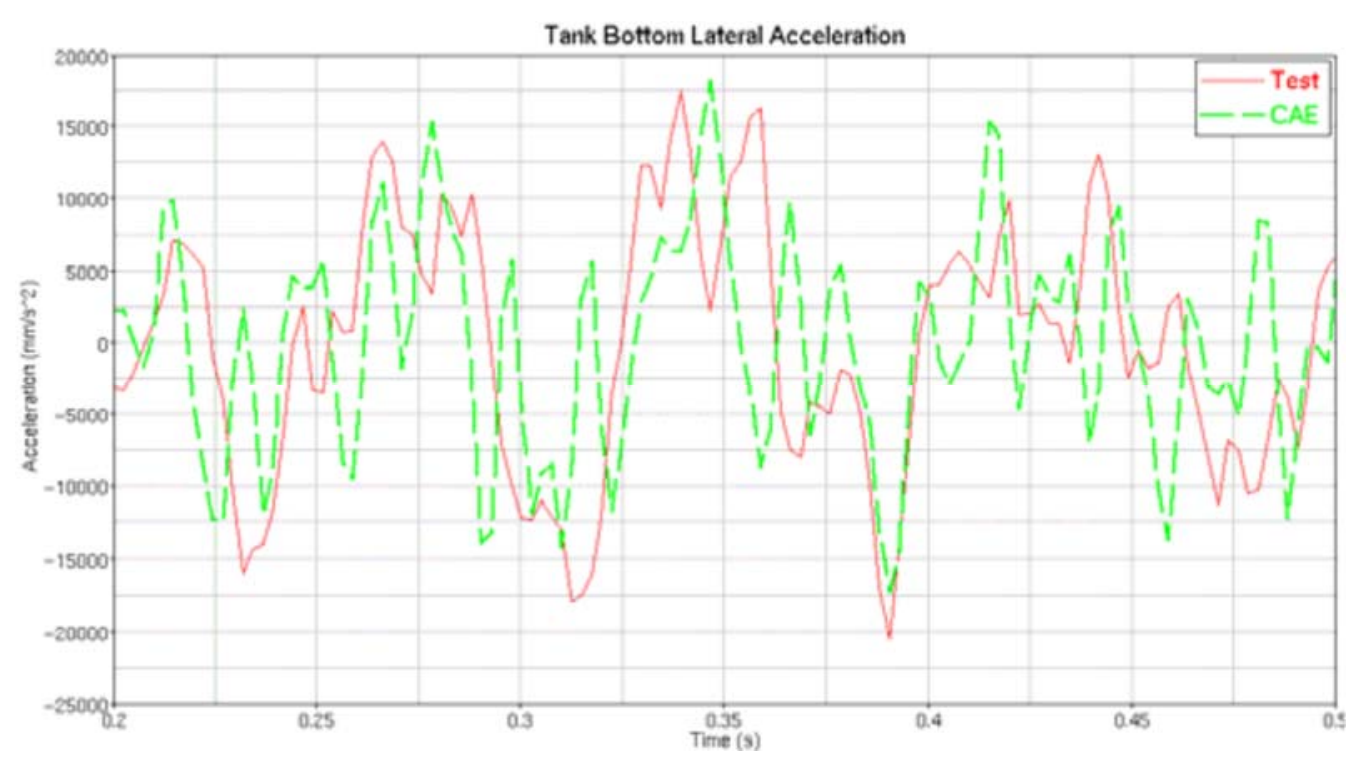

Figure 2-6 Lateral acceleration at the tank bottom [22]
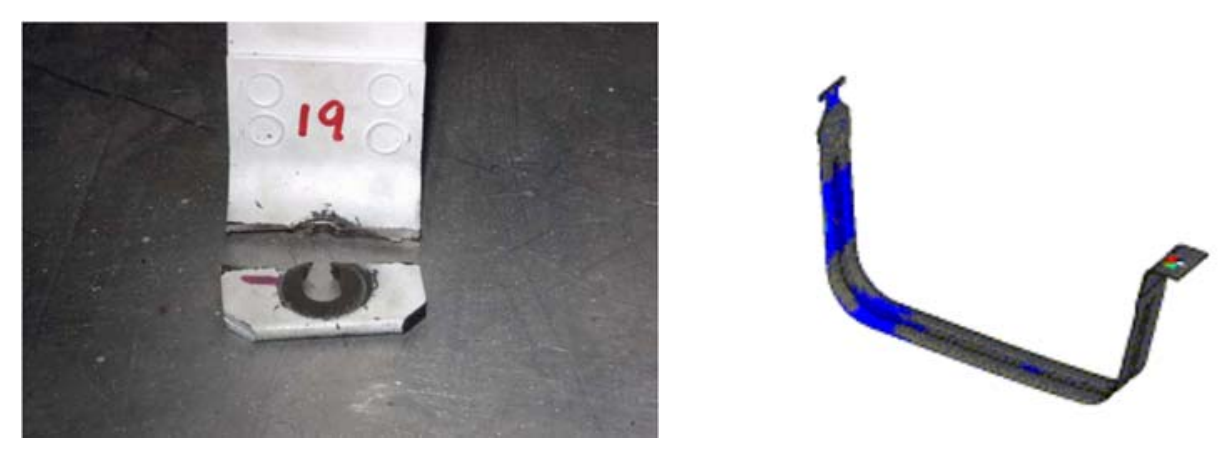

Figure 2-7 Sample \#2, the rear strap cracked due to a loose strap and CAE fatigue prediction [22]

\subsection{Literature Review Summary}

Literature review above shows ALE method is already widely used in some industries, such as Forming Processes, Two-Phase Flows with Phase Change and Heat Transfer of Computer Chips, and Plasma Physics, etc. 
While in auto industry, ALE method is being applied in fuel tank leakage safety simulation with vehicle at high speed impact in the paper by Tang, B. [20]. This ALE method application is relatively too easy because the load input is too simple, an initial velocity, that won't cause too much CPU time to be used.

In the paper by Lin, B., et al., [21], the proving ground load is specifically requested for fuel tank strap, which is not included in the standard testing procedure for full vehicle testing, which means the fuel tank strap load is not available for regular vehicle testing, because the part may have to be cut or modify to locate load cell so that vehicle can't be used again for testing design verification, and load cell installation is very complex and will induce problems. Generally the force time history at any location is generated from vehicle dynamics CAE simulation based on ADAMS model validation through the collected acceleration road testing load.

\subsubsection{Load Cell}

A load cell is a transducer that is used to create an electrical signal whose magnitude is directly proportional to the force being measured [25]. In vehicle testing, it is referred to Strain Gauge Load Cell.

Through a mechanical construction, the force being sensed deforms a strain gauge. The strain gauge measures the deformation (strain) as a change in electrical resistance, which is a measure of the strain and hence the applied forces. A load cell usually consists of four strain gauges in a Wheatstone bridge configuration. Load cells of one strain gauge (quarter bridge) or two strain gauges (half bridge) are also available.

Strain gauge load cells convert the load acting on them into electrical signals. The gauges themselves are bonded onto a beam or structural member that deforms when weight is applied. In most cases, four strain gauges are used to obtain maximum sensitivity and temperature 
compensation. Two of the gauges are usually in tension, and two in compression, and are wired with compensation adjustments. The strain gauge load cell is fundamentally a spring optimized for strain measurement. Gauges are mounted in areas that exhibit strain in compression or tension. The gauges are mounted in a differential bridge to enhance measurement accuracy. When weight is applied, the strain changes the electrical resistance of the gauges in proportion to the load.

Commons issues could be induced as following,

- Mechanical mounting: Friction may induce offset or hysteresis. Wrong mounting may result in the cell reporting forces along undesired axis.

- Overload: If subjected to loads above its maximum rating, the material of the load cell may plastically deform; this may result in a signal offset, loss of linearity, difficulty with or impossibility of calibration, or even mechanical damage to the sensing element (e.g. delamination, rupture).

- Wiring issues: the wires to the cell may develop high resistance, e.g. due to corrosion. Alternatively, parallel current paths can be formed by ingress of moisture. In both cases the signal develops offset (unless all wires are affected equally) and accuracy is lost.

- Electrical damage: the load cells can be damaged by induced or conducted current. Lightning hitting the construction, or arc welding performed near the cells, can overstress the fine resistors of the strain gauges and cause their damage or destruction.

- Nonlinearity: at the low end of their scale, the load cells tend to be nonlinear. This becomes important for cells sensing very large ranges, or with large surplus of load capability to withstand temporary overloads or shocks (e.g. the rope clamps). More points may be needed for the calibration curve. 
In the paper by Qin, P., [7], MFLHUID method is just an approximate method for fuel movement with many assumption.

- This method assumes that the fluid is incompressible and the free surface has zero pressure with no sloshing effects. Also, no viscous (rotational flow) effects exist.

- Compressibility and surface gravity effects are neglected. It is assumed that the important frequency range for the structural modes is above the gravity sloshing frequencies and below the compressible acoustic frequencies. It is further assumed that the density within a volume is constant and no viscous (rotational flow) or aerodynamic (high velocity) effects are present.

- The applied modal transient analysis belongs to linear method which will not consider plastic deformation, even though it takes advantage of linear superposition theory to save computation time.

- The modal transient analysis has less accuracy than direct transient analysis, due to modes truncation.

\subsubsection{Virtual Fluid Mass MFluid Method}

The derivation of MFLUID method is based on the boundary element method. In the boundary element method, point sources $\sigma_{\mathrm{i}}$ are distributed along boundary grids [23]. The known boundary motions are used to solve for $\sigma_{\mathrm{i}}$. Once $\sigma_{\mathrm{i}}$ is known, the motion of the entire field is known as shown in the following equation.

The velocity at grid $\mathrm{i}$ due to these point sources is

$$
\dot{\mathrm{U}}_{\mathrm{i}}=\sum_{\mathrm{j}} \int_{\mathrm{A}_{\mathrm{i}}} \frac{\sigma_{\mathrm{j}} \mathrm{e}_{\mathrm{ij}}}{\left|\mathrm{r}_{\mathrm{i}}-\mathrm{r}_{\mathrm{j}}\right|^{2}} \mathrm{dA} \mathrm{A}_{\mathrm{j}}=\sum_{\mathrm{j}} \mathrm{B}_{\mathrm{ij}} \sigma_{\mathrm{j}}
$$

where $\mathrm{A}$ is area, $\mathrm{r}_{\mathrm{i}}$ is the vector from origin to grid $\mathrm{i}$, and $\mathrm{e}_{\mathrm{ij}}$ is the unit vector from grid $\mathrm{i}$ to j.

In matrix form, 
$\{\dot{U}\}=[B]\{\sigma\}$

The pressure at point $i$ is

$\mathrm{P}_{\mathrm{i}}=\sum_{\mathrm{j}} \int_{\mathrm{A}_{\mathrm{i}}} \frac{\rho \dot{\sigma}_{\mathrm{j}} \mathrm{e}_{\mathrm{ij}}}{\left|\mathrm{r}_{\mathrm{i}} \mathrm{r}_{\mathrm{j}}\right|^{2}} \mathrm{~d} \mathrm{~A}_{\mathrm{j}}=\sum_{\mathrm{j}} \mathrm{C}_{\mathrm{ij}} \dot{\sigma}_{\mathrm{j}}$

where $\rho$ is the fluid density.

The force at point $i$ is

$F_{i}=\int_{A_{i}} P_{i} d A_{i}=\sum_{j} C_{i j} \dot{\sigma}_{j}$

or in matrix form,

$\{\mathrm{F}\}=[\mathrm{D}]\{\dot{\sigma}\}=[\mathrm{D}][\mathrm{B}]^{-1}\{\ddot{\mathrm{u}}\}=[\mathrm{M}]\{\ddot{\mathrm{u}}\}$

This yield the mass matrix

$[\mathrm{M}]=[\mathrm{D}][\mathrm{B}]^{-1}$

which represents the fuel and fuel-to-tank interaction.

The mass matrix will be included in the global finite element mass matrix to solve the dynamic equation. It only depends on the fluid density and the fluid boundary geometry. In the CAE model, only the wetted surface needs to be defined. No physical elements of the fluid need to be modeled.

\subsubsection{Modal Transient Response}

Modal transient response is an alternate approach to computing the transient response of a structure [24]. This method uses the mode shapes of the structure to reduce the size, uncouple the equations of motion (when modal or no damping is used), and make the numerical integration more efficient. Since the mode shapes are typically computed as part of the characterization of the structure, modal transient response is a natural extension of normal modes analysis.

As a first step in the formulation, transform the variables from physical coordinates $\{\mathrm{u}\}$ to modal coordinates $\{\xi\}$ by 


$$
\{u(t)\}=[\varnothing]\{\xi(t)\}
$$

The mode shapes $[\varnothing]$ are used to transform the problem in terms of the behavior of the modes as opposed to the behavior of the grid points. Equation (2-7) represents an equality if all modes are used; however, because all modes are very rarely used, the equation usually represents an approximation.

To proceed, temporarily ignore the damping, resulting in the equation of motion

$$
[M]\{\ddot{u}(t)\}+[K]\{\varnothing\}\{\xi(t)\}=\{P(t)\}
$$

If the physics coordinates in terms of the modal coordinates (Eq. (2-7) is substituted into Eq. (2-8)), the following equation is obtained:

$$
[M][\varnothing]\{\ddot{\xi}(t)\}+\{K\}[\varnothing]\{\xi(t)\}=\{P(t)\}
$$

This is now the equation of motion in terms of the modal coordinates. At this point, however, the equations remain coupled.

To uncouple the equations, premultiply by $[\varnothing]^{\mathrm{T}}$ to obtain

$$
[\varnothing]^{T}[M][\varnothing]\{\ddot{\xi}(t)\}+[\varnothing]^{T}\{K\}[\varnothing]\{\xi(t)\}=[\varnothing]^{T}\{P(t)\}
$$

Where:

$[\varnothing]^{T}[M][\varnothing]=$ modal $($ generalized) mass matrix

$[\varnothing]^{T}\{K\}[\varnothing]=$ modal (generalized) stiffness matrix

$[\varnothing]^{T}\{P(t)\}=$ modal force vector

The final step uses the orthogonality property of the mode shapes to formulate the equation of motion in terms of the generalized mass and stiffness matrices that are diagonal matrices. These matrices do not have off-diagonal terms that couple the equations of motion. Therefore, in this form, the modal equations of motion are uncoupled. In this uncoupled form, the equations of motion are written as a set on uncoupled SDOF systems as 
$m_{i} \ddot{\xi}_{i}(t)+k_{i} \xi_{i}(t)=p_{i}(t)$

Where:

$m_{i}=\mathrm{i}$-th modal mass

$k_{i}=\mathrm{i}$-th modal stiffness

$p_{i}=\mathrm{i}$-th modal force

Once the individual modal responses $\xi_{i}(t)$ are computed, physical responses are recovered as the summation of the modal responses

$$
\{u(t)\}=[\varnothing]\{\xi(t)\}
$$

Since numerical integration is applied to the relatively small number of uncoupled equations, there is not as large a computational penalty for changing $\Delta t$ as there is in direct transient response analysis. However, a constant $\Delta \mathrm{t}$ is still recommended.

The stresses (or strains) are also in this format, with the $[\varnothing]_{i}$ representing modal stresses (or strains). This format matches that of linear superposition of stresses in quasi-static fatigue

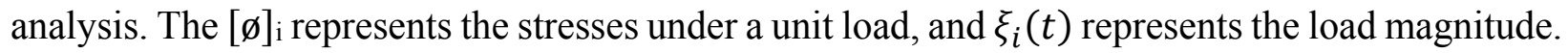

Therefore, once the modal stresses and modal coordinate time history are calculated, the same superposition method as in quasi-static fatigue analysis can be used for dynamic fatigue calculation. The computation time for a modal stress and a modal coordinate time history calculation is much less than that of a direct stress or strain time history calculation. This is because the number of $\xi_{i}(t)$ is usually much smaller than the number of the degrees of freedom in the CAE model.

To further improve accuracy, the bases formed by the mode shapes can be expanded to include the static deformed shapes (residual flexibility). They are the deformed shapes of the 
system under the load of each hydraulic input, balanced by inertia. Including them in the bases can increase stress or strain accuracy by capturing the "quasi-static" part of the dynamic responses.

\subsubsection{Modal versus Direct Transient Response}

Some general guidelines can be used in selecting modal transient response analysis versus direct transient response analysis [24]. These guidelines are summarized in Table 2-1.

Table 2-1 Modal versus Direct Transient Response [24]

\begin{tabular}{|l|c|c|}
\hline & $\begin{array}{l}\text { Modal Transient } \\
\text { Response }\end{array}$ & $\begin{array}{l}\text { Direct Transient } \\
\text { Response }\end{array}$ \\
\hline Small Model & $\mathrm{x}$ & $\mathrm{x}$ \\
\hline Large Model & $\mathrm{x}$ \\
\hline Few Time Steps & $\mathrm{x}$ & \\
\hline Many Time Steps & & $\mathrm{x}$ \\
\hline High Frequency Excitation & & $\mathrm{x}$ \\
\hline Normal Damping & & $\mathrm{x}$ \\
\hline Higher Accuracy & $\mathrm{x}$ & $\mathrm{x}$ \\
\hline Initial Conditions & & \\
\hline
\end{tabular}

In general, larger models may be solved more efficiently in modal transient response because the numerical solution is a solution of a smaller system of uncoupled equations. This result is certainly true if the natural frequencies and mode shape were computed during a previous stage of the analysis. Using Duhamel's integral to solve the uncoupled equations is very efficient even for very long duration transients. On the other hand, the major portion of the effort in a modal transient response analysis is the calculation of the modes. For large systems with a large number of modes, this operation can be as costly as direct integration. This is especially true for highfrequency excitation. To capture high frequency response in a modal solution, less accurate highfrequency modes must be computed. For small models with a few time steps, the direct method may be the most efficient because it solves the equations without first computing the modes. The 
direct method is more accurate than the modal method because the direct method is not concerned with mode truncation. Table 2-1 provides a starting place for evaluating which method to use. Many additional factors may be involved in the choice of a method, such as contractual obligations or local standards of practice. 


\section{CHAPTER 3 GOVERNING EQUATIONS OF ARBITRARY LAGRANGIAN-EULERIAN}

\subsection{ALE Form of Conservation Equations}

In Lagrangian formulation, the observer follows material points. In Eulerian formulation, the observer looks at fixed points in space. In Arbitrary Lagrangian-Eulerian formulation, the observer follows moving points in space.

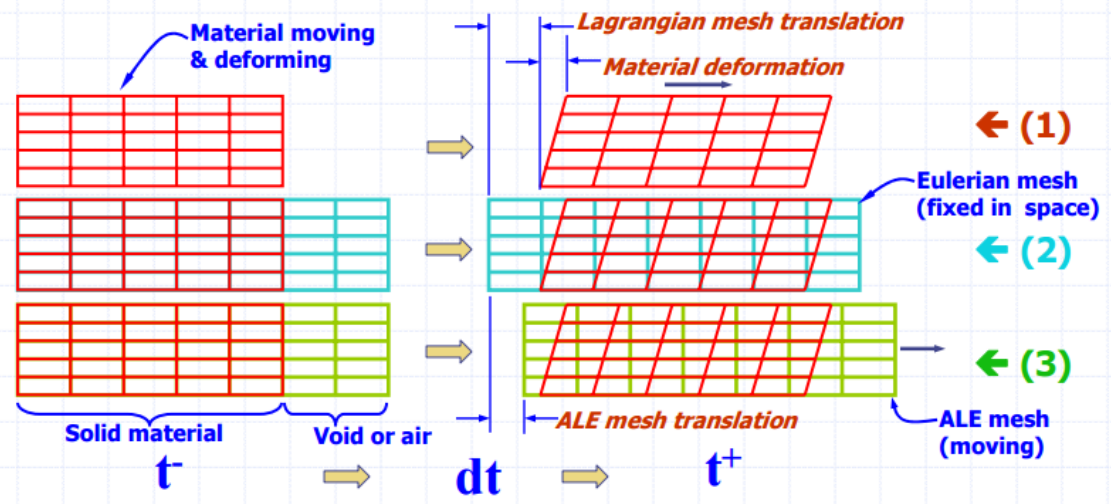

Figure 3-1 Lagrangian mesh, Eulerian mesh, and ALE mesh comparison [29]

At any location in space $\mathrm{x}$ and time $\mathrm{t}$, one material point is identified by its space coordinates $\mathrm{X}$ at time 0 and one grid point identified by it's coordinates $\xi$ at time 0 .

$$
\begin{gathered}
X=\Phi(X, t)=\hat{\Phi}(\xi, t) \text { Jacobians } \neq 0 \\
\text { LAG ALE }
\end{gathered}
$$

Where $\Phi$ is a function of space and time, representing a physical property.

The continuum equations for multi-material ALE method are [30]:

1. Mass conservation equation

$$
\left.\frac{\partial \rho}{\partial t}\right|_{x}+\nabla_{x} \cdot(\rho \boldsymbol{u})=0
$$


2. Moment conservation equation

$$
\left.\rho \frac{\partial u}{\partial t}\right|_{x}+\rho \mathbf{u} \cdot \nabla_{x} \boldsymbol{u}=\nabla_{x} \cdot \mathbf{S}+\rho \mathbf{g}
$$

3. Total energy conservation equation

$$
\left.\rho \frac{\partial e_{0}}{\partial t}\right|_{x}+\rho \mathbf{u} \cdot \nabla_{x} e_{0}=\nabla_{x} \cdot\left(k \nabla_{x} \mathrm{~T}\right)+\mathbf{S} \cdot \nabla_{x} \boldsymbol{u}+\rho \mathbf{g} \cdot \mathbf{u}
$$

Where $t$ is time, $\rho$ is density, $\mathbf{S}$ is the Cauchy stress tensor, $\mathbf{g}$ is the gravity vector, $e_{0}$ is the specific internal energy, $k$ is the thermal conductivity and $T$ is the temperature. The above equations assume that gravity is the only body force, the thermal conductivity is isotropic, and that there is not internal generation of heat.

In ALE method, the structure is modeled as Lagrangian, and the fluid domain as ALE or Eulerian. The grid/mesh has an arbitrary motion, but the material goes through. The grid velocity term is added in a compressible Navier Stokes solver for viscous fluids as shown in Figure 3-2.

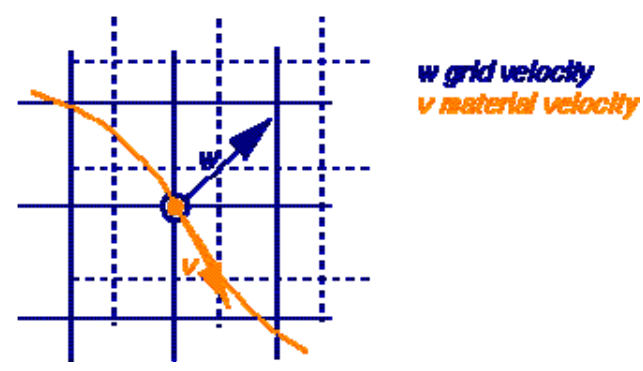

Figure 3-2. Grid velocity and material velocity in compressible Naiver Stokes equation [27] 


\subsection{Arbitrary Lagrangian-Eulerian Update Procedure ${ }^{1}$}

The objective of Arbitrary Lagrangian-Eulerian (ALE) formulations is to stop the calculation when the mesh is distorted, smooth the mesh, and remap the solution from the distorted mesh to the smooth mesh.

\subsubsection{Overall Flow of ALE Steps}

In ALE formulations, two basic strategies to upgrade mesh following Lagrangian time step are "remap” or "advection” step. The advection step is to move the nodes only a small fraction of the characteristic lengths of the surrounding element, including decide and move the boundary nodes and interior nodes, calculate the transport of the element-centered variables, and calculate the momentum transport and update the velocity.

\subsubsection{Mesh Smoothing Algorithms}

ALE formulation applies the algorithms for moving the mesh relative to the material to control the range of the problems. Winslow developed the equipotential method in 1963, which has been proven to be very successful in a wide variety of problems. This "Equipotential” zoning method is making a structured mesh for finite different or finite element calculation by using the solutions of Laplace equations as the mesh lines, and as well, is applied to smooth selected points in an unstructured three-dimensional mesh provided that it is at least locally structured. The other well-known applied mesh smoothing algorithms are Simple Averaging, Kikuchi’s Algorithm, Surface Smoothing, and Combining Smoothing Algorithm.

\subsubsection{Advection Algorithm}

In 1977 Van Leer developed “The van Leer MUSCL scheme” to calculate the values of the solution variables (i.e., density, internal energy, the stress tensor and the history variables) in

\footnotetext{
${ }^{1}$ LS-DYNA Theory Manual, (C) 2016 Livermore Software Technology Corporation. Reprinted with permission.
} 
the transport fluxes to achieve second order accurate monotonic results. To calculate the momentum transport, in 1992, Benson implemented a method which is validated without dispersion and monotonicity issue on regular mesh. Currently the applied methods are Advection Methods in One Dimension, The Donor Cell Algorithm, The Van Leer MUSCL Algorithm, Advection Methods in Three Dimension, etc. For example, in Advection Methods in Three Dimension, the algorithms could be Advection of the Nodal Velocities, The Staggered Mesh Algorithm, The SALE Algorithm, The Half Index Shift Algorithm, Three-Dimensional Momentum Advection Algorithms.

3.2.4 The Manual Rezone

For a problem involving large deformations, it may appear that a mesh stops working after some iterations, in which a manual rezoning capability is needed to implement. The general procedure is to 1) interrupt the calculation when the mesh is no longer acceptable, 2) generate a new mesh using the current material boundaries, 3) remap the solution from the old mesh to the new mesh, and 4) restart the calculation 


\section{CHAPTER 4 ARBITRARY LAGRANGIAN-EULERIAN APPLICATION EXAMPLES}

To validate ALE method feasibility in vehicle simulation, four simple examples are performed to see how good the ALE method will be in the animation, respectively in fuel sloshing simulation, explosive simulation for plate crack initiation and propagation and military ground vehicle roll over [29].

\subsection{Fuel Tank Sloshing}

This is an Eulerian sloshing model with air and fluid in the upper and lower part respectively. Due to dissipation in the advection it still has an energy loss, but viscous behavior has been reduced by using *CONTROL_HOURGLASS command. The fluid is modeled as solid ALE with Elastic material properties under $1 \mathrm{~g}$ vertical load.

This sliced sheet is prescribed by the horizontal motion with a timedependent impulse. The fluid domain is modeled with the *MAT_ELASTIC_FLUID and Solid ALE elements, whereas the gaseous domain is modeled with the *MAT_NULL and Solid ALE elements with the Eulerian formulation without smoothing of Arbitrary Lagrangian-Eulerian description.

The fluid and gaseous domains are incorporated in the Eulerian elements by *ALE_MULTI MATERIAL_GROUP. All fluid domains are discretized by the *SECTION_SOLID_ALE, using the element type 11 - 1 point integration solid ALE with multimaterial. 


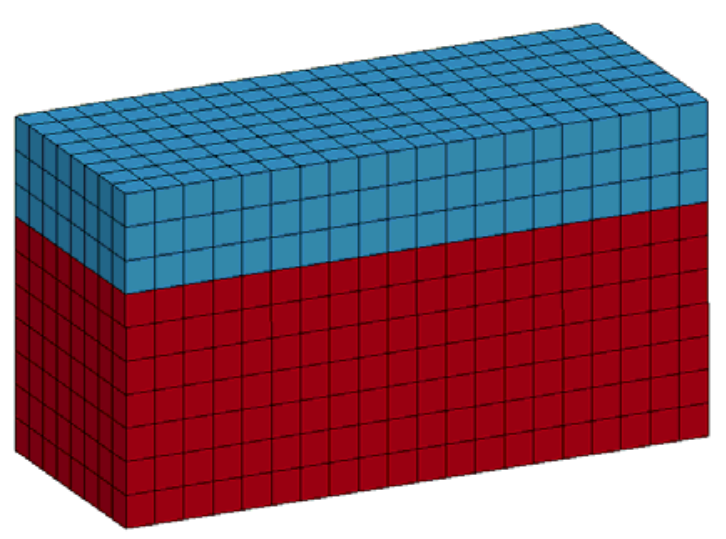

Figure 4-1 Sloshing block with air and fluid

Table 4-1 below is the dimension of sloshing tank, which is shown in Table 4-1.

Table 4-1 Sloshing tank dimension

\begin{tabular}{|c|c|c|}
\hline Length $(\mathrm{mm})$ & Width $(\mathrm{mm})$ & Height $(\mathrm{mm})$ \\
\hline 200 & 80 & 110 \\
\hline
\end{tabular}

Energy curves in Figure 4-2 show the energy loss in the sloshing.

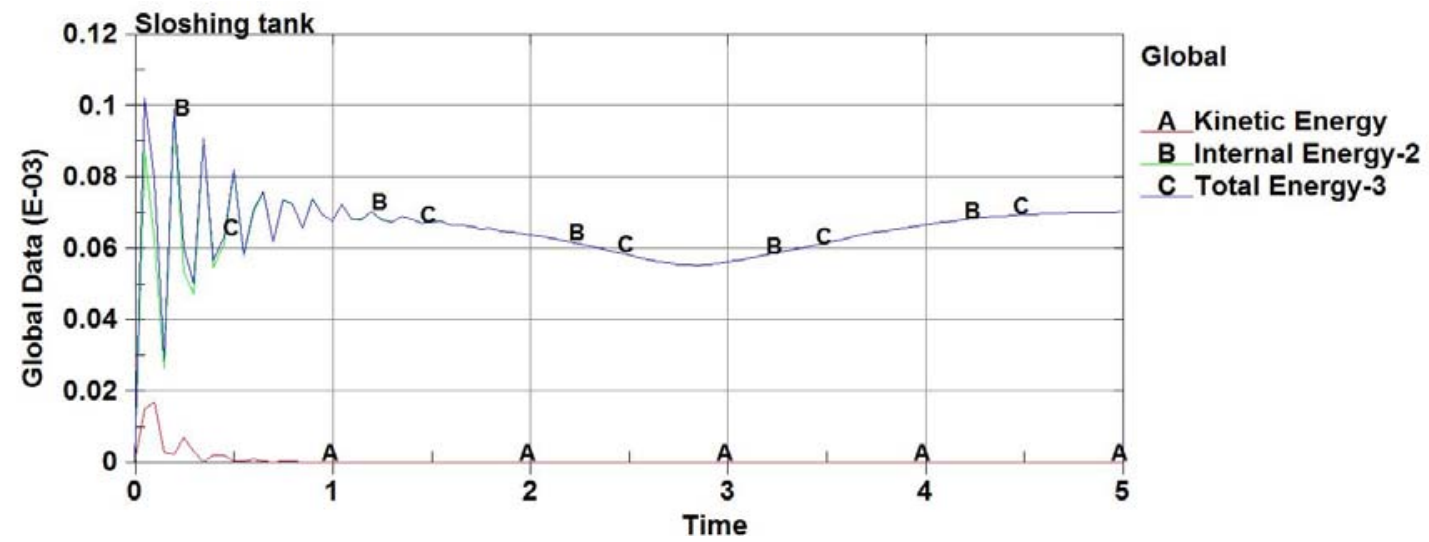

Figure 4-2 Energy curves 
Internal pressure flow animations in Time T1, T2, T3, and T4 are shown in Figure 4-3. To show more clear animation for internal pressure change alongside the time, pressure iso-surfaces are listed in Figure 4-4. This example shows how ALE method is applied in sloshing simulation.

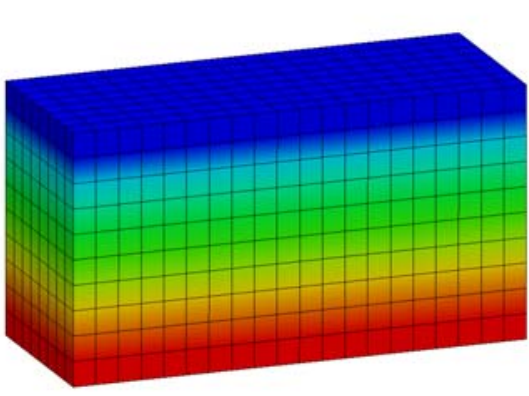

Time T1

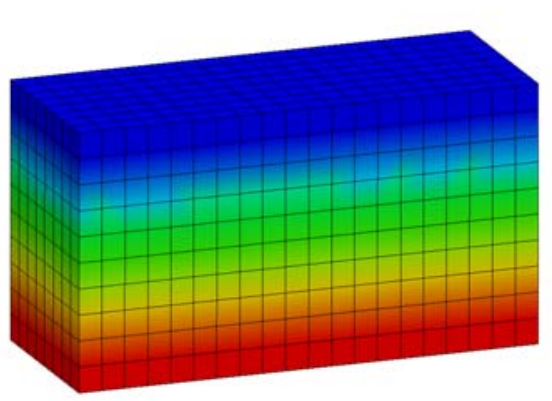

Time T3

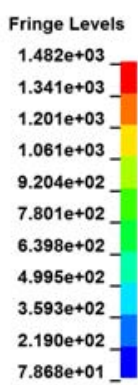

$$
\begin{gathered}
\text { Fringe Levels } \\
1.123 \mathrm{e}+03 \\
1.014 \mathrm{e}+03 \\
9.054 \mathrm{e}+02 \\
7.967 \mathrm{e}+02 \\
6.880 \mathrm{e}+02 \\
5.793 \mathrm{e}+02 \\
4.706 \mathrm{e}+02 \\
3.619 \mathrm{e}+02 \\
2.532 \mathrm{e}+02 \\
1.445 \mathrm{e}+02 \\
3.576 \mathrm{e}+01
\end{gathered}
$$

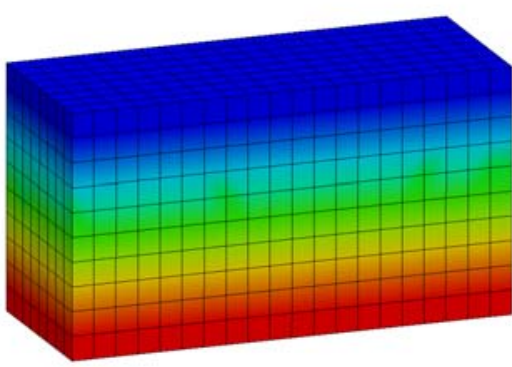

Fringe Levels

$1.042 \mathrm{e}+03$

$9.402 \mathrm{e}+02$

$8.387 \mathrm{e}+02$

$7.371 \mathrm{e}+02$

$6.356 \mathrm{e}+02$

$5.340 \mathrm{e}+02$

$4.324 \mathrm{e}+02$

$3.309 \mathrm{e}+02$

$2.293 \mathrm{e}+02$

$1.278 \mathrm{e}+02$

$2.623 \mathrm{e}+01$ ]

Figure 4-3 Sloshing pressure animation 


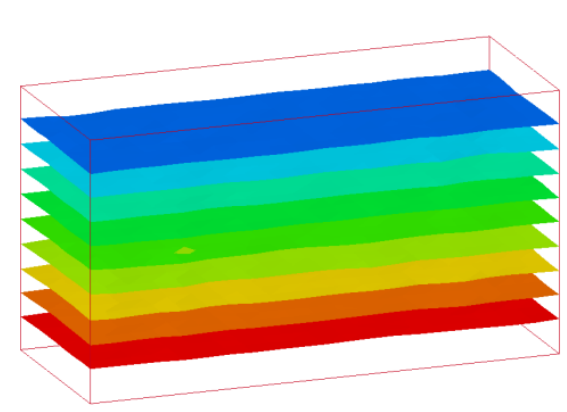

Time T1

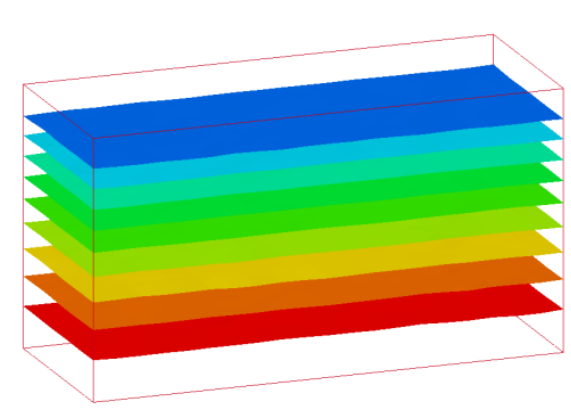

Time T3
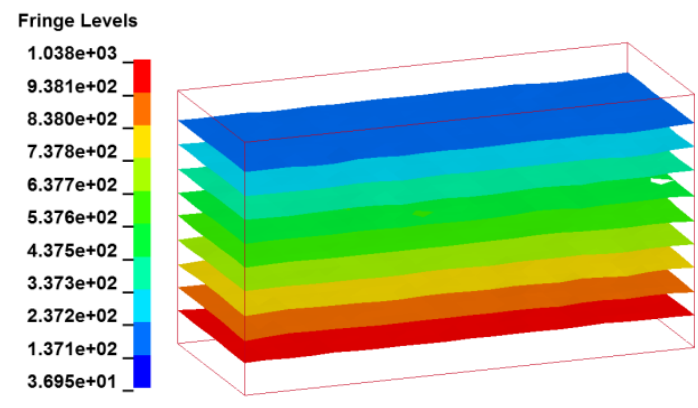

Time T2
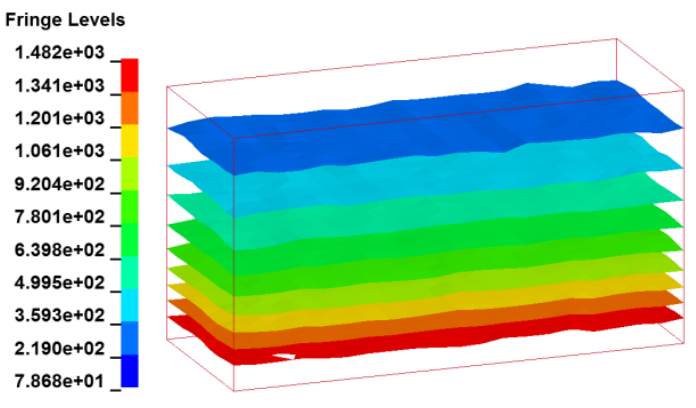

Time T4

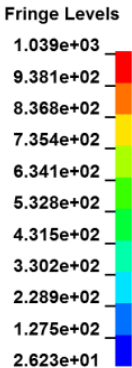

Fringe Levels $5.412 \mathrm{e}+02$ $4.863 \mathrm{e}+02$ $4.315 \mathrm{e}+02$ $3.767 \mathrm{e}+02$ $3.218 \mathrm{e}+02$ $2.670 \mathrm{e}+02$ $2.122 \mathrm{e}+02$ $1.573 \mathrm{e}+02$ $1.025 \mathrm{e}+02$ $4.768 \mathrm{e}+01$ $-7.153 \mathrm{e}+00]$

Figure 4-4 Sloshing pressure iso-surface animation

\subsection{Plate Deformation under Explosive}

In the multi-material ALE approach the plate is modeled enclosed in a layer of void (vacuum) with explosive on the bottom center, as shown in Figure 4-5. The dimension is in Table 4-2, and material properties for two different material in Table 4-3 in comparison, high strength steel and Titanium. 


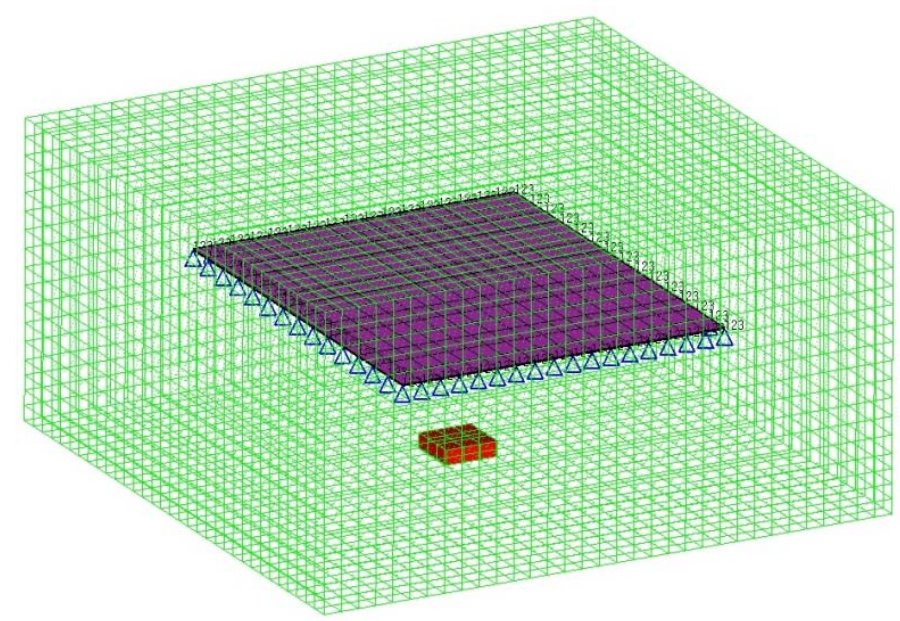

Figure 4-5 Plate in vacuum

Table 4-2 Blast plate dimension

\begin{tabular}{|c|c|c|}
\hline Length $(\mathrm{cm})$ & Width $(\mathrm{cm})$ & Thickness $(\mathrm{cm})$ \\
\hline 140 & 113 & 0.54 \\
\hline
\end{tabular}

Table 4-3 Blast plate material properties

\begin{tabular}{|c|c|c|c|c|c|c|}
\hline & $\begin{array}{c}\text { Density } \\
\left(\mathrm{g} / \mathrm{cm}^{3}\right)\end{array}$ & $\begin{array}{c}\text { Young's Modulus } \\
(\mathrm{mbar})\end{array}$ & Poisson's Ratio & $\begin{array}{c}\text { Yield Strength } \\
(\mathrm{mbar})\end{array}$ & $\begin{array}{c}\text { Tangent Modulus } \\
(\mathrm{mbar})\end{array}$ & Failure Strain \\
\hline High Strength Steel & 7.83 & 2.07 & 0.29 & 0.01329 & 0.06547 & 0.1133 \\
\hline Titanium & 4.4 & 1.14 & 0.3 & 0.008707 & 0.01697 & 0.125 \\
\hline
\end{tabular}

The pressure propagation of explosive material in the fluid core is transferred to other parts and the Fluid Solid Interaction (FSI) is taken into account by the Arbitrary Lagrangian-Eulerian method (ALE) and fluid-structure-coupling.

The explosive is modeled by the *SECTION_SOLID_ALE with *MAT_HIGH_EXPLOSIVE_BURN and *EOS_JWL. The outermost fluid layer of the box 
is modeled by the *SECTION_SOLID_ALE with *MAT_NULL and *EOS_LINEAR_POLYNOMIAL.

The coupling between the structure (Lagrangian) and the fluid (Eulerian) is done by a FSIalgorithm, *CONSTRAINED_LAGRANGE_IN_SOLID.

The command *ALE_MULTI-MATERIAL_GROUP is used to specify that elements containing materials of the same group are treated as single material elements. In this example, part 1 and part 2 are under the same multi-material group.

Displacement animation can show clear different reaction between two plates in exact same timing, as shown in Figure 4-6, 4-7. The high strength steel plate shows buckling, while Titanium doesn't.

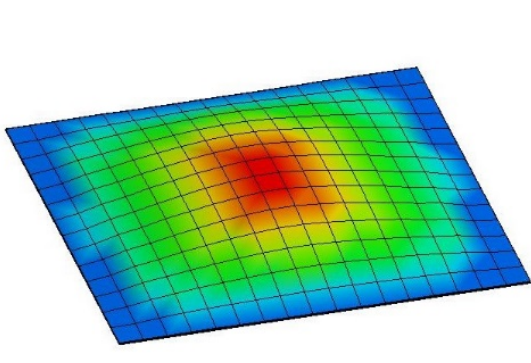

Time T1

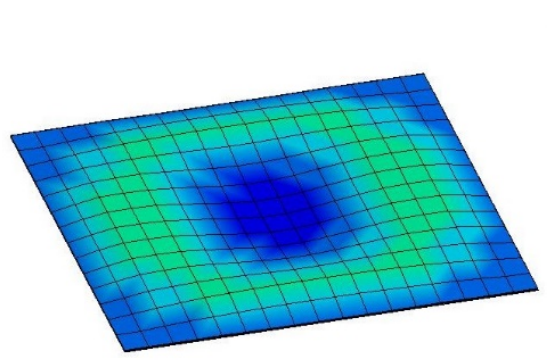

Time T3
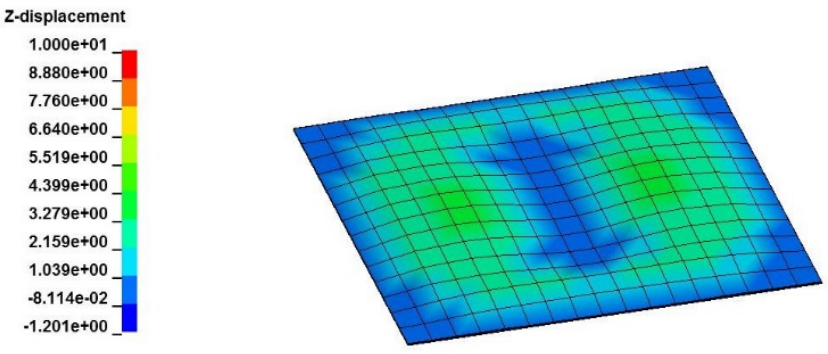

Z-displacement $1.000 \mathrm{e}+01$ $8.880 \mathrm{e}+00$ $7.760 \mathrm{e}+00$ $6.640 \mathrm{e}+00$ $5.519 \mathrm{e}+00$ $4.399 \mathrm{e}+00$ $3.279 \mathrm{e}+00$ $2.159 \mathrm{e}+00$ $1.039 \mathrm{e}+00$ $-8.114 \mathrm{e}-02$ $-1.201 \mathrm{e}+00$

$$
\begin{gathered}
\text { Z-displacement } \\
1.000 \mathrm{e}+01 \\
8.880 \mathrm{e}+00] \\
7.760 \mathrm{e}+00 \\
6.640 \mathrm{e}+00 \\
5.519 \mathrm{e}+00 \\
4.399 \mathrm{e}+00 \\
3.279 \mathrm{e}+00 \\
2.159 \mathrm{e}+00 \\
1.039 \mathrm{e}+00 \\
-8.114 \mathrm{e}-02 \\
-1.201 \mathrm{e}+00
\end{gathered}
$$

Time T2

Figure 4-6 Displacement of high strength steel 


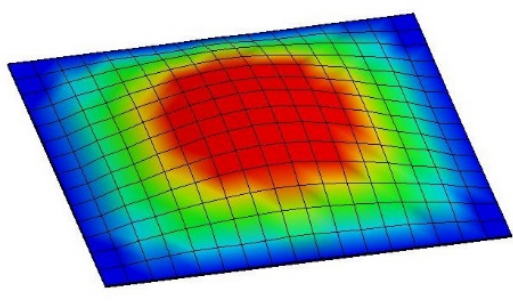

Time T1

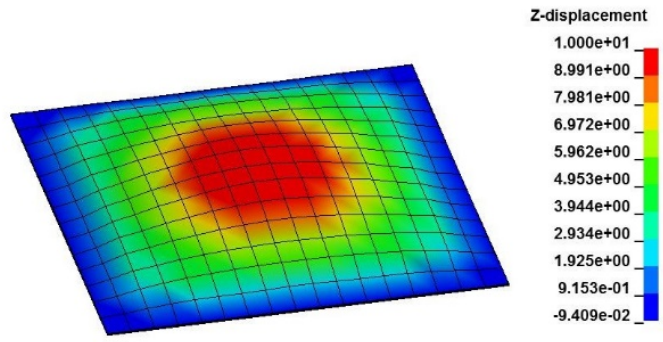

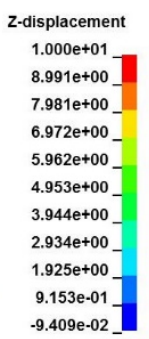

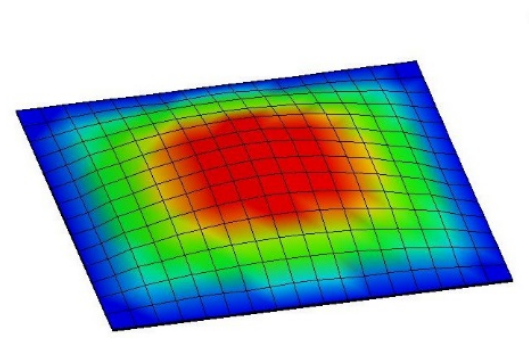

2-displacement $1.000 \mathrm{e}+01$ $8.991 \mathrm{e}+00$ $7.981 \mathrm{e}+00$ $6.972 \mathrm{e}+00$ $5.962 \mathrm{e}+00$ $4.953 \mathrm{e}+00$ $3.944 \mathrm{e}+00$ $2.934 \mathrm{e}+00$ $1.925 \mathrm{e}+00$ $9.153 \mathrm{e}-01$ $-9.409 \mathrm{e}-02$

Time T2

Time T3

Figure 4-7 Displacement of Titanium plate

Center points displacement curves plotted in Figure 4-8 shows the center area buckling of steel plate, and effective plastic strain curves in Figure 4-9 shows permanent deformation formed in both plates.

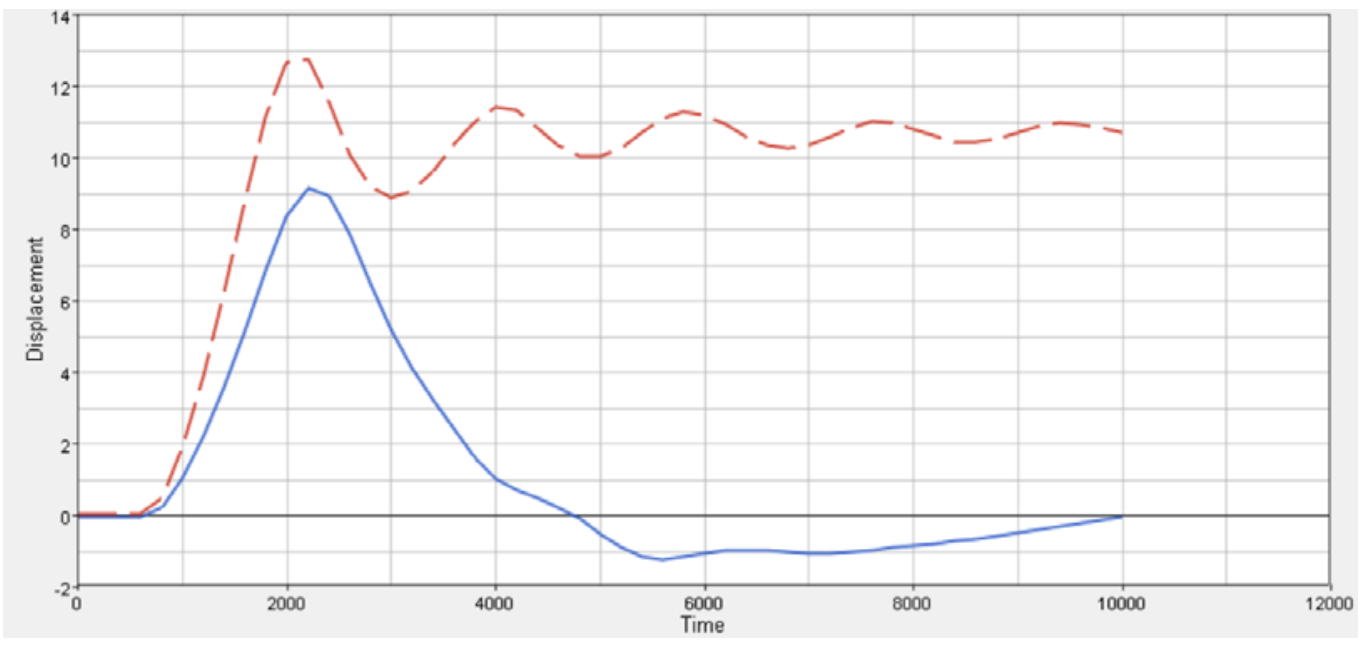

Figure 4-8 Displacement of center point of two plates 


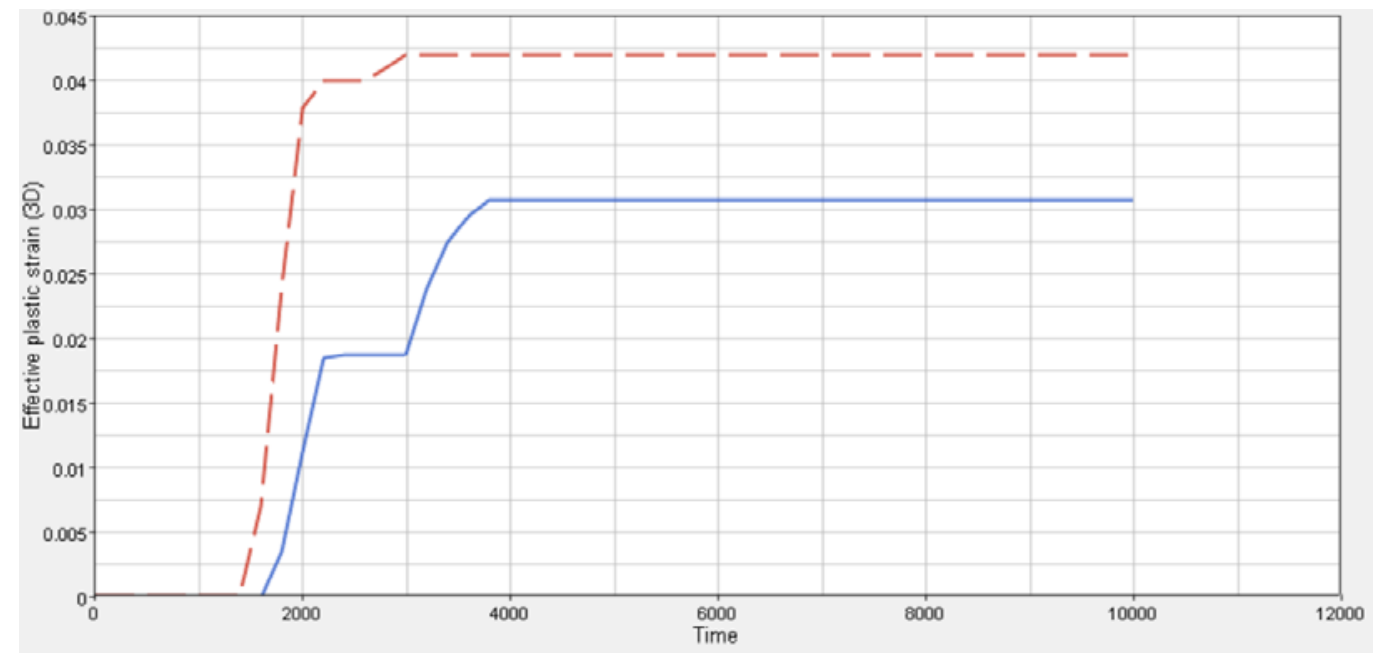

Figure 4-9 Effective plastic strain of center point of two plates

Energy curves are plotted in Figure 4-10 for high strength steel plate and Figure 4-11 for Titanium plate, which show the total energy lost in blast.

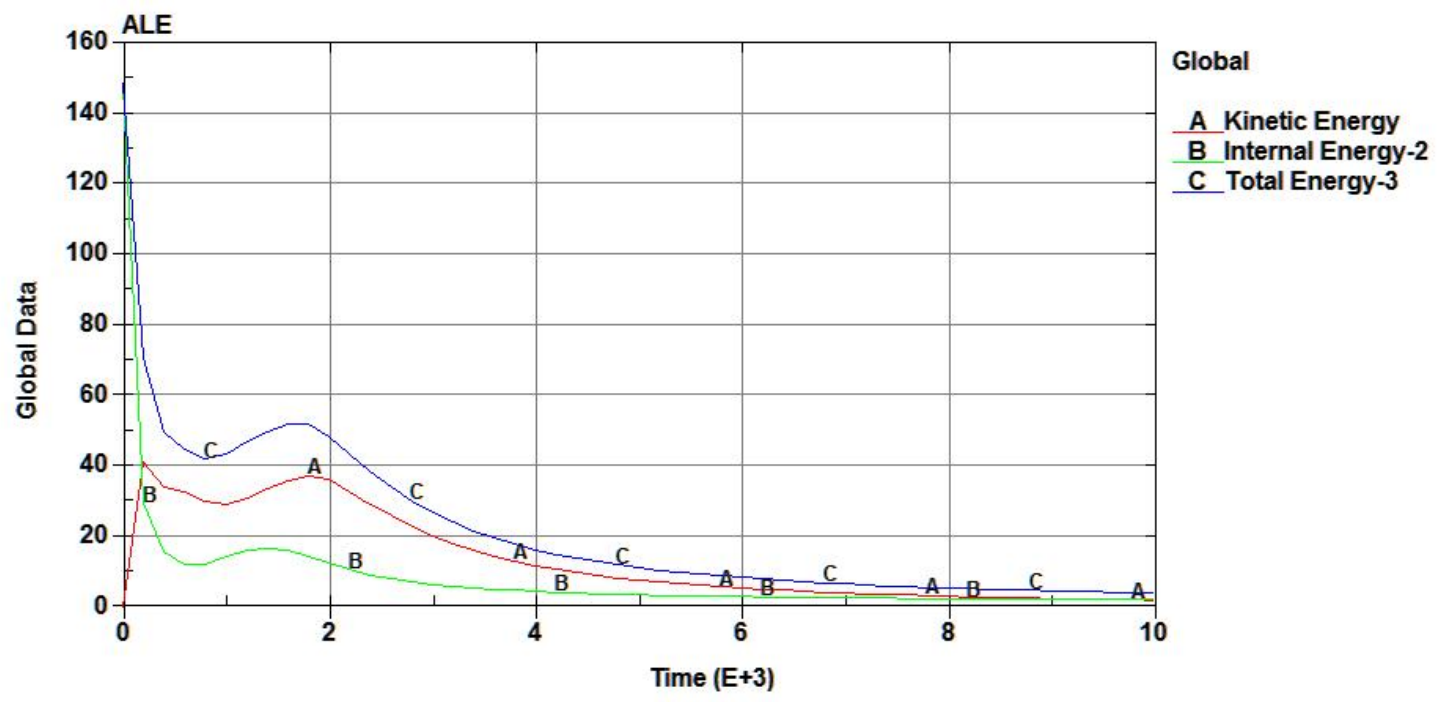

Figure 4-10 Energy curves for high strength 


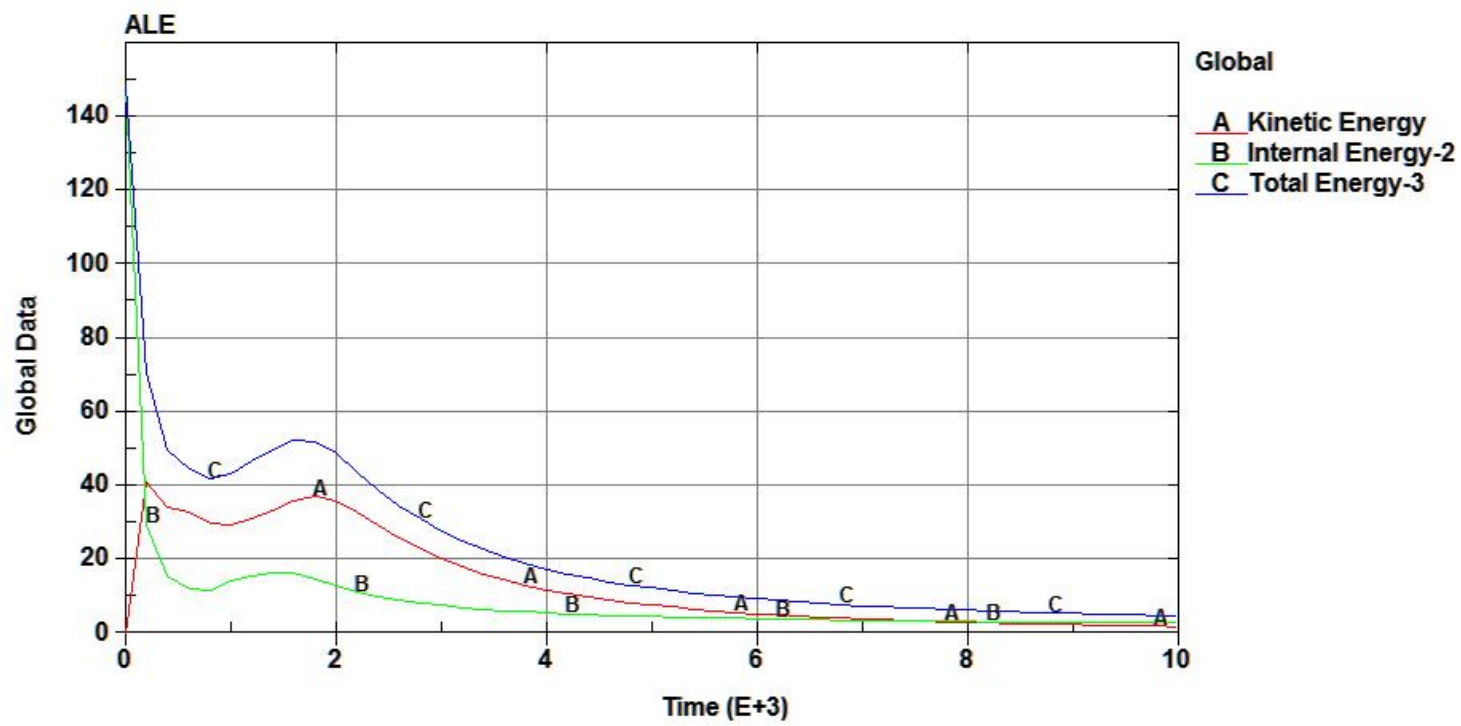

Figure 4-11 Energy curves for Titanium steel

\subsection{High Strength Steel Plate Crack Initiation and Propagation under Explosive}

To study the crack behavior of high strength steel, the mass of explosive is increased by 10 times. With the element corrosion is defined in the keyword with ultimate failure strain at $11.33 \%$, crack initiation and propagation is shown in the animation with effective plastic strain legend, as seen in Figure 4-12. 


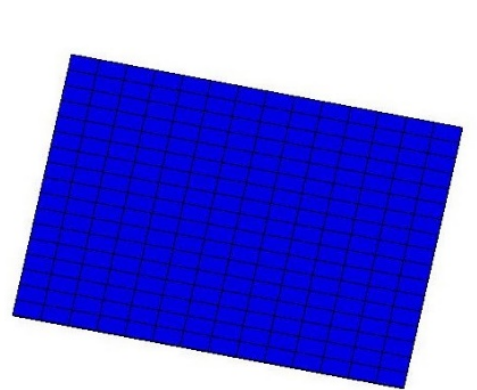

Time T1

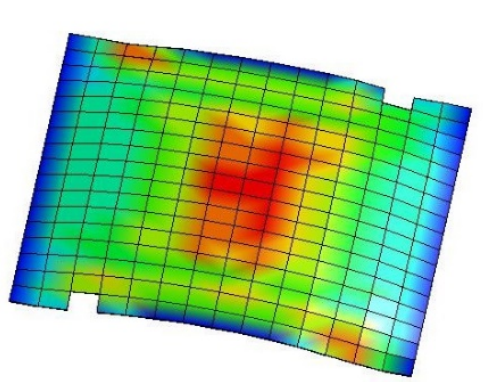

Time T3

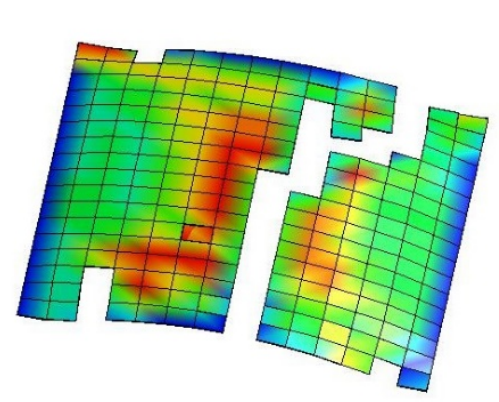

Time T5
Effective Plastic Strain $0.000 \mathrm{e}+00$

$0.000 \mathrm{e}+00$ $0.000 \mathrm{e}+00$ $0.000 \mathrm{e}+00$

$0.000 \mathrm{e}+00$

$0.000 \mathrm{e}+00$

$0.000 \mathrm{e}+00$

$0.000 \mathrm{e}+00$

$0.000 \mathrm{e}+00$

$0.000 \mathrm{e}+00$

$0.000 \mathrm{e}+00]$

Efrective Plastic Strain 4.237e-02

$3.813 \mathrm{e}-02$

3.389e-02

2.966e-02

2.542e-02

2.118 -02

1.695e-02

1.271e-02

$8.473 \mathrm{e}-03$

4.237e -03

$0.000 \mathrm{e}+00]$

Effective Plastic Strain 4.320e-02

$3.888 \mathrm{e}-02$ ]

$3.456 \mathrm{e}-02$

$3.024 \mathrm{e}-02$

2.592e-02

$2.160 \mathrm{e}-02$

$1.728 \mathrm{e}-02$

$1.296 \mathrm{e}-02$

$8.640 \mathrm{e}-03$

$4.320 \mathrm{e}-03$

$0.000 \mathrm{e}+00]$

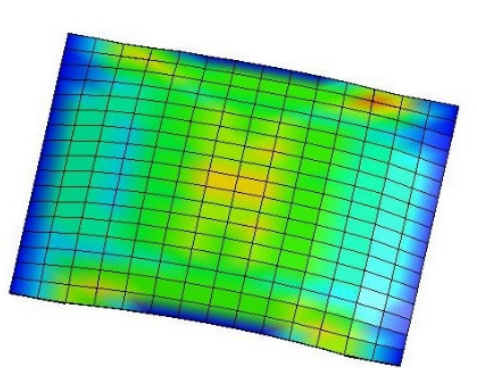

Time T2
Effective Plastic Strain 4.096e- 02 3.687e-02 ] 3.277e-02 2.867e-02 2.458e-02 $2.048 \mathrm{e}-02$ 1.638 -02 1.229e-02 8.192e-03 4.096e-03 $0.000 \mathrm{e}+00]$

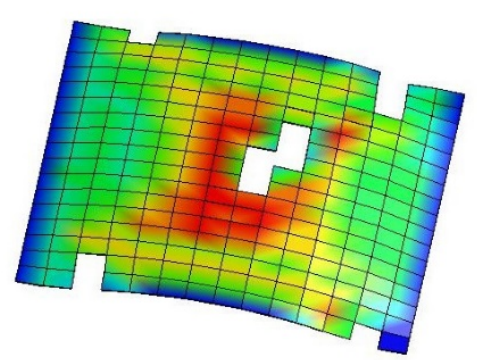

Effective Plastic Strain 4.319e-02 3.887e-02] $3.456 \mathrm{e}-02$

$3.024 \mathrm{e}-02$ 2.592e-02 2.160e-02 $1.728 \mathrm{e}-02$ $1.296 \mathrm{e}-02$ $8.639 \mathrm{e}-03$ 4.319e-03 $0.000 \mathrm{e}+00$

\section{Time T4}

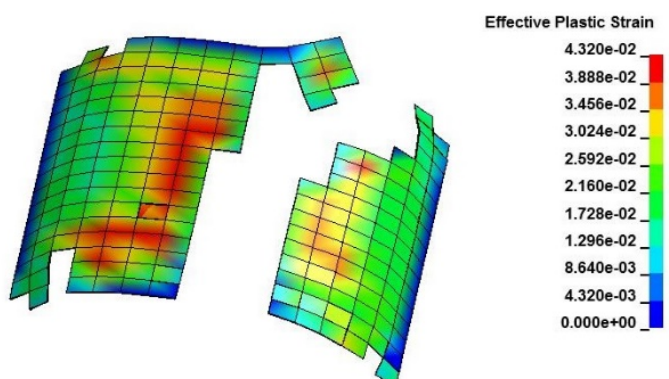

Time T6

Figure 4-12 Crack initiation and propagation in high strength steel

Energy curves are plotted in Figure 4-13 for high strength steel plate that shows the energy lost. 


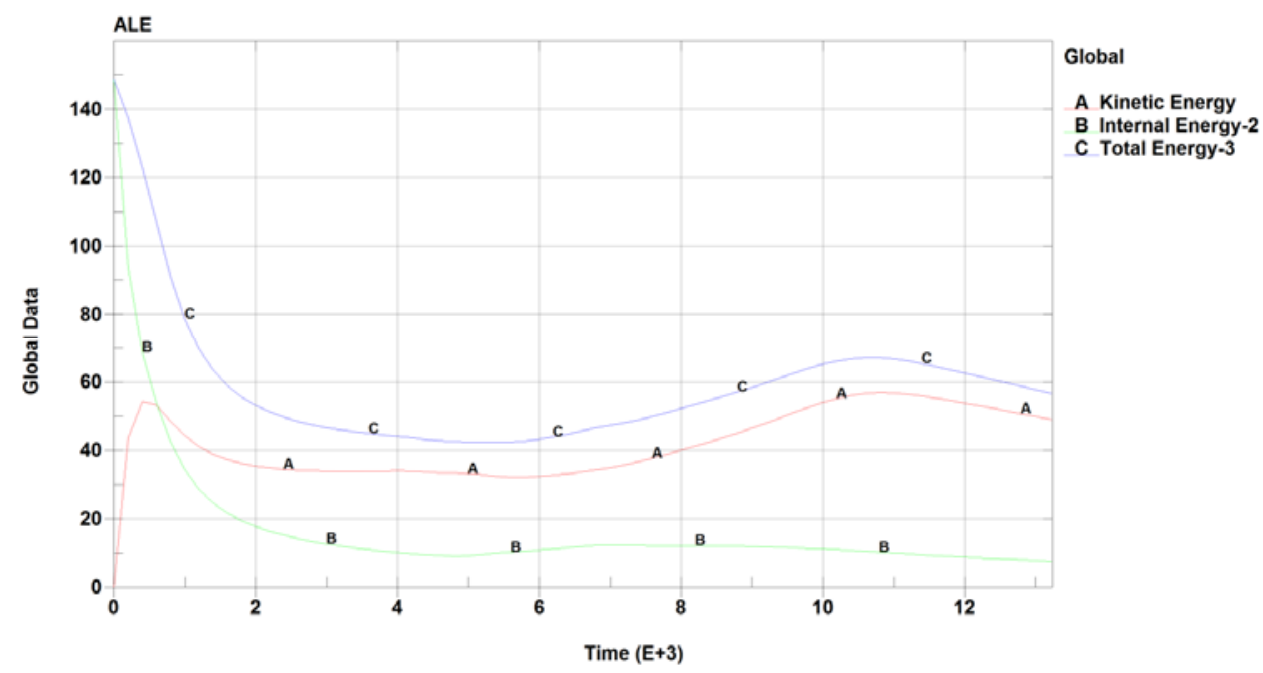

Figure 4-13 Energy curves for high strength steel plates in crack initiation and propagation

\subsection{Vehicle Rollover by Mine Blast}

A vehicle is modeled as a solid enclosed in vacuum with explosive under right front tire.

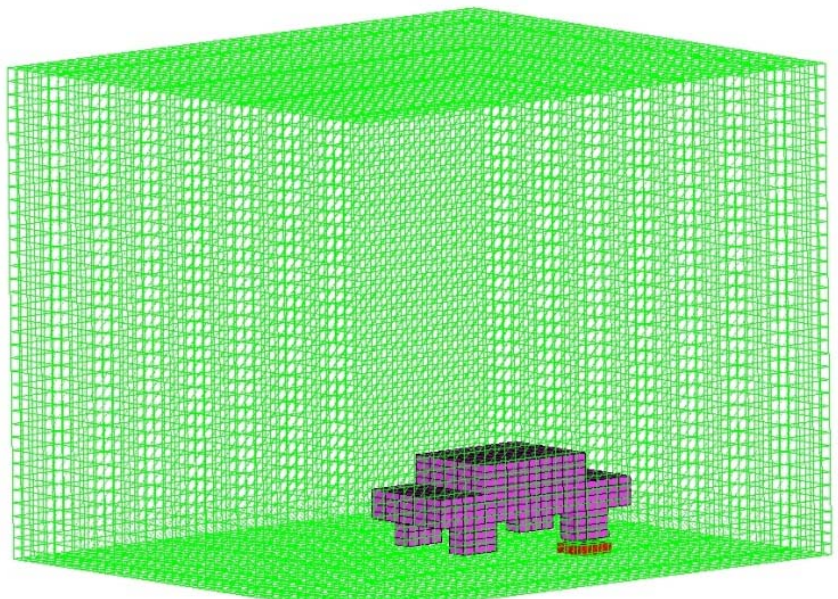

Figure 4-14 Vehicle in vacuum with explosive under tire

Rollover animation are shown in Figure 4-15 at timing T1, T2, T3, T4, and T5. 


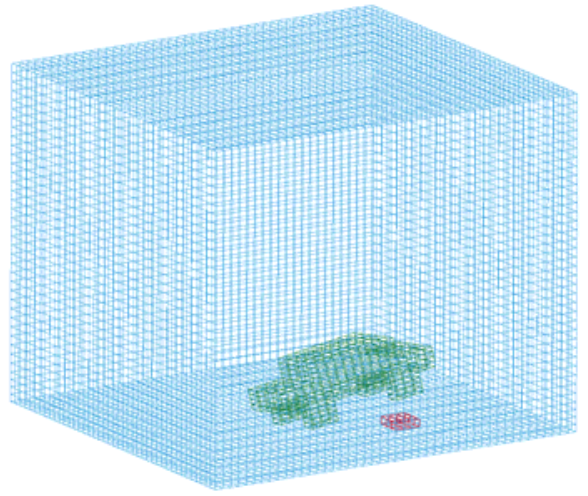

Time T1

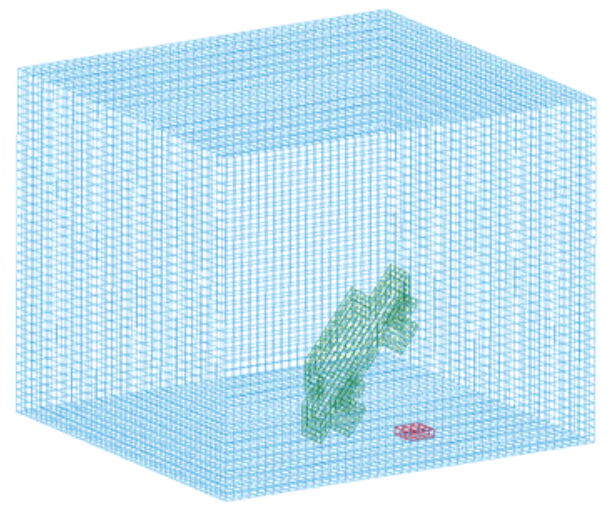

Time T3

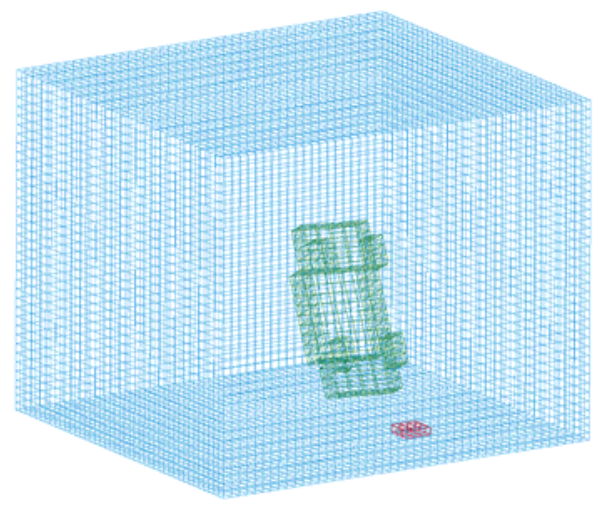

Time T5

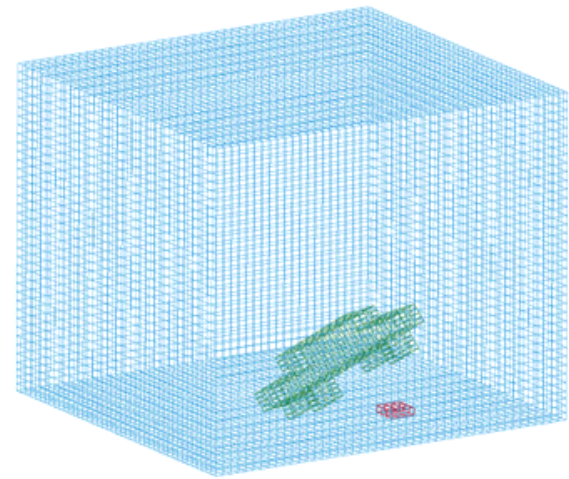

Time T2

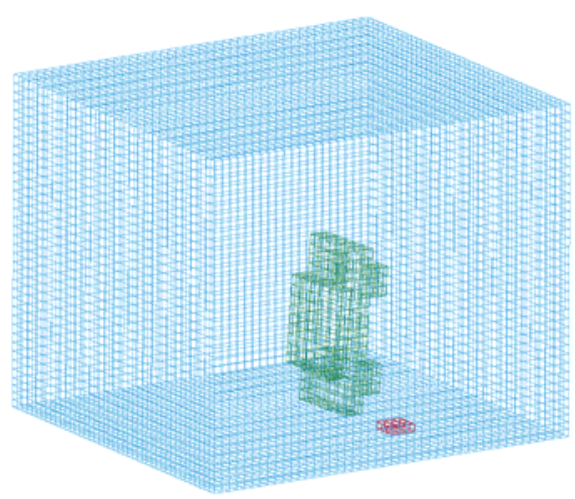

Time T4

Figure 4-15 Vehicle rollover by explosive blast 
Energy curves exhibited greatly decrease in the whole period.

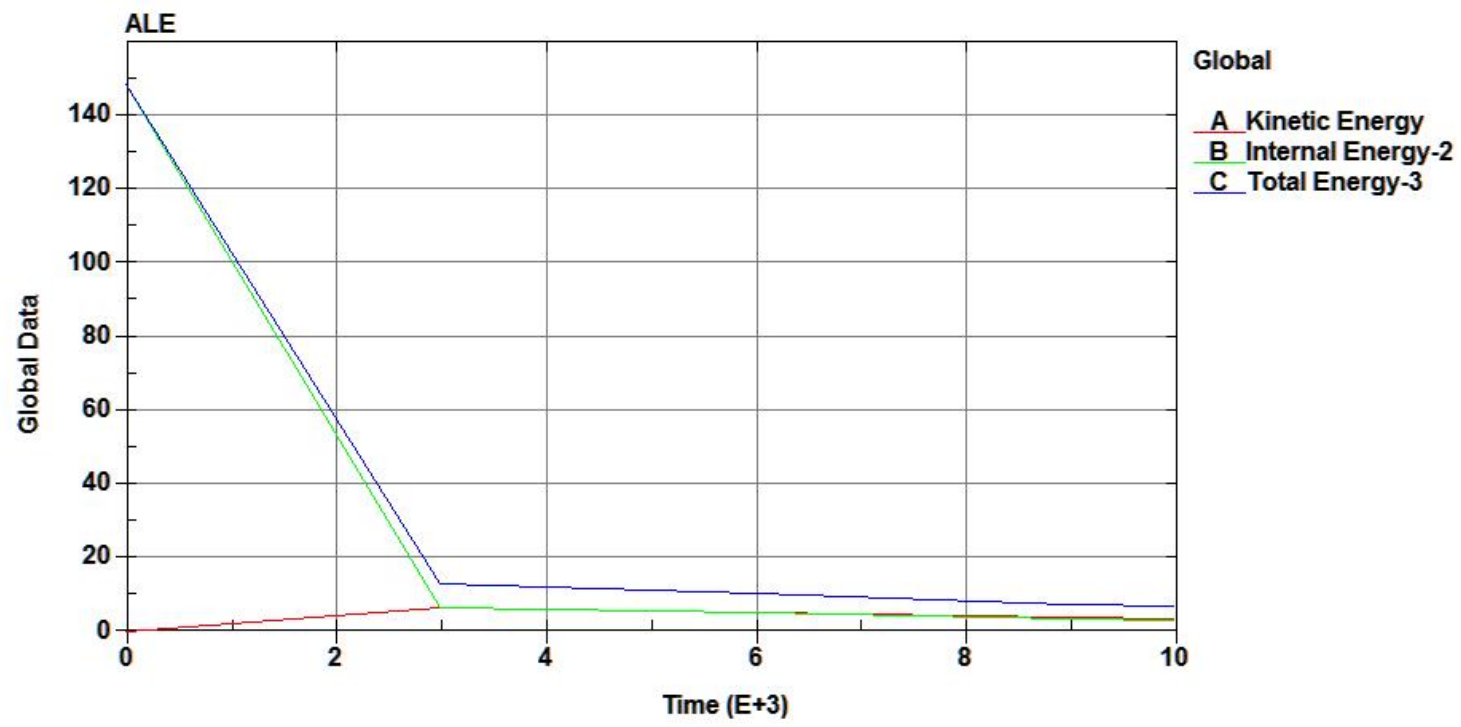

Figure 4-16 Energy curves in explosive

\subsection{Conclusion}

ALE method in LS-DYNA is an efficient tool for analyzing large deformation processes to simulate multi-material interaction, i.e., sloshing in the tank with fluid and air interaction, and explosive in the air with structure and air interaction. With the failure criterion defined in the model, crack initiation and propagation can be animated too.

It should note that the model size with ALE method should be kept minimum to avoid large CPU time due to the complicated algorithm in the LS-DYNA code, which is the limitation for ALE application. In the next example of fuel tank simulation under proving ground condition, modeling skill will be shown how to simplified model to make ALE method feasible and achieve better result. 


\section{CHAPTER 5 ARBITRARY LAGRANGIAN-EULERIAN METHOD IN FUEL TANK STRAP SIMULATION UNDER PROVING GROUND CONDITION}

In automotive CAE durability analysis, simulation of dynamic stress and fatigue life of fuel tank straps is a complex problem. Typically a fuel tank is held with fuel tank straps. Its movement lies in the domain of nonlinear large rotation dynamics. Moreover, the sloshing behavior in the fuel tank makes the problem even more intricate.

The objective of this study is to investigate the advantage of ALE method in simulating fuel sloshing through fuel tank and fuel tank strap movement under proving ground conditions using the nonlinear large rotation dynamic method with RADIOSS, a commercial code. After the stress distribution of the fuel tank strap is achieved, a commercial fatigue code, nCode DesignLife, is used to predict the fatigue life of the fuel tank straps. The analyses have accurately predicted the crack initiation sequence and locations in the fuel tank straps, and show good correlation with test. The utilization of this method can give design direction to minimize the iteration of lab testing and expedite the design period.

\subsection{Analytical Methods Introduction}

Due to inertia, the sloshing phenomenon in the fuel tank occurs when a vehicle is accelerating or decelerating on road surfaces. This effect plus the movement of the fuel tank will result in a pressure change between the straps and the tank. This time varying pressure load will then induce stresses in the straps, and will lead to strap fatigue. To understand the stress pattern and the fatigue life of the straps in this highly nonlinear system has been a challenge for fuel engineers.

CAE methods for fuel tank simulation that are currently used in the automotive industry can be summarized into two categories: linear methods and nonlinear methods. 
In the linear area, there are three commonly used methods: 1. common mass method, 2. concentrated mass method, and 3. fluid volume properties definition method. In the common mass method, the density of the tank wall is adjusted to account for the mass of the tank and fuel. In the concentrated mass method, a concentrated mass element is used to model the fuel at the C.G. of the fuel. This mass is connected to the fuel tank through weighted motion constraints. In the fluid volume properties definition method, the element envelope that contains the volume of the incompressible fuel is given a fuel material property to generate a virtual mass. These three methods give satisfactory results in small displacement environments, but cannot predict the structural responses in large rotation situations.

For nonlinear problems, the following three methods are commonly used in fuel tank simulation:

\subsubsection{Common Mass Nonlinear Method}

The fuel is combined into the fuel tank by adjusting the fuel tank density so the model has the equivalent mass of fuel tank and fuel. Nonlinear material properties and contact surfaces are defined in the model. The advantage of this method is that the fuel tank is allowed large rotations and the analysis is easy to set up without extra modeling effort for fuel. The disadvantage is the missing sloshing effect of the fuel.

\subsubsection{Arbitrary Lagrangian-Eulerian (ALE) Method}

As introduced in Chapter 3, in Lagrangian formulation, the observer follows material points. In Eulerian formulation, the observer looks at fixed points in space. In Arbitrary Lagrangian-Eulerian formulation, the observer follows moving points in space. 
In ALE method, the structure is modeled as Lagrangian, and the fluid domain as ALE or Eulerian. The grid/mesh has an arbitrary motion, but the material goes through. The grid velocity term is added in a compressible Navier Stokes solver for viscous fluids.

High quality solid elements are required for this method. Less CPU time will be required if the tank doesn't deform very much.

\subsubsection{Smooth Particles Hydrodynamics (S.P.H.) Method}

The SPH method can simulate phenomena like tank penetration, fuel leakage, and fuel tank high velocity, but requires much more CPU time [31]. The method uses nodes to represent the mesh in calculation. Due to the modeling difficulty and quite extensive CPU time usage, SPH method is not worthy to be adopted in this study for comparison.

\subsubsection{Fatigue Method}

DesignLife, a commercial software developed by nCode International, estimates fatigue life from finite element analysis result [32]. DesignLife takes stress or strain data from a finite element calculation as input, and combines this information together with the details of the time varying loads, and material cyclic properties. From this combination it estimates the fatigue damage for each supplied node or element on the FE model.

The elastic-plastics method with uniaxial strain life fatigue theory is used in this analysis. First, the total strain life time history from the RADIOSS stress analysis results is input to the program in FORTRAN. The top membrane strain and bottom membrane strain are compared according to strain rate, then the higher strain rate history are composed. Second, the material cyclic stress strain curve is used to track stress strain history, and strain cycles and the mean stress are output. 
$\varepsilon=\sigma / E+\left(\sigma / K^{\prime}\right)^{1 / n^{\prime}}$

1)

Where

$K^{\prime}=\sigma_{f} /\left(\varepsilon_{f}\right)^{n^{\prime}}$ is Cyclic Strength Coefficient

$n^{\prime}=\mathrm{b} / \mathrm{c}$ is Cyclic Strain Hardening Exponent

$\sigma_{f}$ is Fatigue Strength Coefficient

$\varepsilon_{f}$ is Fatigue Ductility Coefficient

$\mathrm{b}$ is Fatigue Strength Exponent

c is Fatigue Ductility Exponent

Third, the Smith-Watson-Topper equation is used to get damage from each strain cycle.

$\sigma_{\max } \varepsilon_{a}=\left(\sigma_{f}^{\prime 2} / E\right)\left(2 N_{f}\right)^{2 b}+\sigma_{f}^{\prime} \varepsilon_{f}^{\prime}\left(2 N_{f}\right)^{b+c}$

Where

$N_{f}$ is the Number of Reversals to Failure and $2 N_{f}$ is the number of reversals to fatigue

$\sigma_{\max }=\sigma_{m}+\sigma_{a}$ is Maximum Stress of the Cycle

$\sigma_{m}$ is Mean Stress

$\sigma_{a}$ is Stress Amplitude

$\varepsilon_{a}$ is Maximum Strain of the Cycle

Finally, Miner's rule is used to sum the damage of all the cycles.

5.1.5 Equation of Motion Explicit Time Integration

The equation of equilibrium governing the dynamic response of the system are [33]

$[M]\{x\}+[C]\{x\}+[\ddot{K}]\{x\}^{\cdot}=\left\{R^{\operatorname{ext}}(t)\right\}$ 


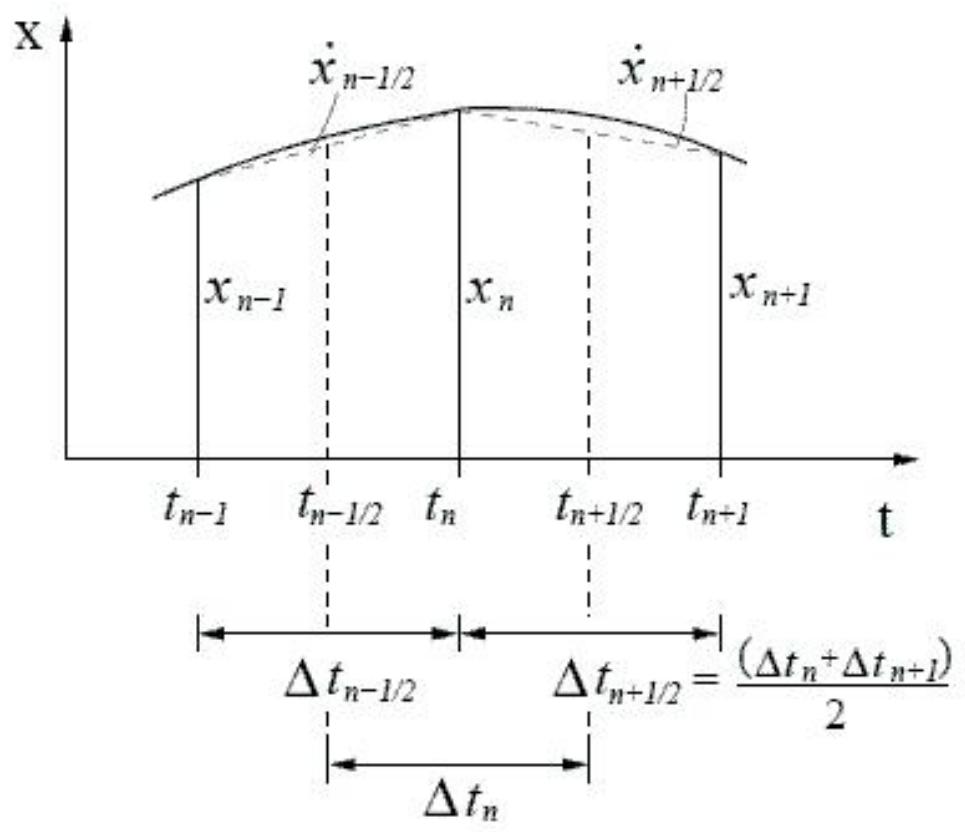

Figure 5-1 Explicit time integration [33]

Velocity: $\dot{x}_{n+\frac{1}{2}}=\dot{x}_{n-\frac{1}{2}}+\Delta t_{n} \ddot{x}_{n}$

Displacement: $x_{n+1}=x_{n}+u_{\text {stat }}$

Initial conditions: $x_{0}=\bar{x}_{0}+\Delta t_{n+\frac{1}{2}}$

Original geometry $\bar{x}_{0}+$ initial displacements $u_{\text {stat }}$ from static loading + forces $F_{0}^{\text {int }}$ from initial strains

$v_{0}=\frac{1}{2}\left(\dot{x}_{-\frac{1}{2}}+\dot{x}_{\frac{1}{2}}\right) \quad$ initial velocity

This yields: $\ddot{x}_{0}=M^{-1}\left(P_{0}+P_{\text {stat }}-F_{0}^{\text {int }}\left(u_{\text {stat }}\right)-C v_{0}\right)$

$\dot{x}_{\frac{1}{2}}=v_{0}+\frac{1}{2} \Delta t_{0} \ddot{x}_{0}$

With $P_{\text {stat }}-F_{0}^{\text {int }}\left(u_{\text {stat }}\right)=0$ 


\subsubsection{Signal Processing in MATLAB}

Accelerometers are the most frequently used transducers to measure the vibration responses of the structures [34]. Sometimes it is necessary to retrieve the measures signals in the form of velocities and displacements. While it is quite easy to extract the information on the frequency components and corresponding amplitudes of the velocities and displacements of the measured acceleration signals, it is not an easy task to retrieve the time history of the structural responses in the form of velocities and displacements. There are two methods, in general, to convert the acceleration signal to the displacement signal. One is directly integrating the acceleration signal in the time domain. The other is dividing the Fourier transformed acceleration by the scale factor of $-\omega^{2}$ and taking the inverse Fourier transform of it. It is well known that to have better resolution in time domain, one has to compromise with the coarse resolution in frequency domain and vice versa with given number of sampling points. Therefore, with a fixed resolution in time and frequency domain, converting high frequency signals in the time domain and converting low frequency signals in frequency domain will produce biased errors.

1. Converting Acceleration to Displacement Using Time Domain Method

When the time history of the acceleration is available and the Nyquist frequency of the digitization is much higher than the highest frequency components of the signal, the direct integration of the acceleration in time domain retrieves reasonably accurate time history of the displacement.

The relationship between the frequency of the signal and the Nyquist frequency of the measurement is that the higher the signal frequency, the worse the retrieved displacement signal. The second source of error comes from the fact that there is no information available on the initial conditions involved with each integration scheme. 
Uncertain value of initial velocity will produce a DC component during the successive integration of the conversion.

2. Converting Acceleration to Displacement Using Fast Fourier Transformation Method

If the frequency components of the measured acceleration are relatively high compared to the Nyquist frequency, the frequency domain method should be used. From the properties of the Fourier transform of the integrals, the Fast Fourier Transform of the velocity and displacement will drop off velocity and displacement levels by $3 \mathrm{~dB}$ and $6 \mathrm{~dB}$ per doubling the frequency, and therefore, high frequency components in the velocity and displacement can be less than the low frequency noise components after conversion. The frequency domain method works well with the acceleration signal measured without leakage, in which cases the magnitude of the significant frequency components is much bigger than noncontributing frequency components. In practice, structural response consists of both high and low frequency components signals and leakage always happens in the measurement.

To convert a measured acceleration signal with minimum error in frequency domain, there must be some procedure to minimize the effects of the scaling factor on the low frequency components, because the conversion factor on the low frequency components can become significant after the Fourier components are divided by the scale factor of $-\omega^{2}$. Most of the measured structural accelerations are either steady state responses or decaying transient responses. The steady state responses consists of single frequency sinusoidal signals, and found in study, zero-padding can reduce the effect of the conversion factor and provide satisfactory results.

Zero padding before a DFT/FFT is a computationally efficient method of interpolating a large number of points. Therefore, zero padding the data before you obtain the DFT can often 
increase the frequency resolution and thus improve the amplitude estimate. To avoid the spectrum leakage, Hanning windows is usually applied in signal processing as moderate window [35].

$w(n)=0.5\left(1-\cos \left(\frac{2 \pi n}{N}\right)\right), 0 \leq n \leq N$

\subsection{Experimental Set up}

The fuel tank and the strap fatigue life test as set up in the lab is shown in Figure 5-2. The fuel tank and straps are mounted on a partial truck frame supported by four vertical hydraulic actuators. In addition, three horizontal actuators are used to form a total of seven-channel inputs to the test buck, accounting for all six degrees of freedom. The excitation inputs are recorded from a vehicle going through proving ground surfaces. This time history data is for structural analysis purposes and does not represent the real customer use condition. In this particular set up, the original cross member forward of the tank is removed from the frame and is replaced with one straight, box section, cross member. This is more convenient for the installation of the hydraulic actuators. A remotely located computer controls the hydraulic actuators.

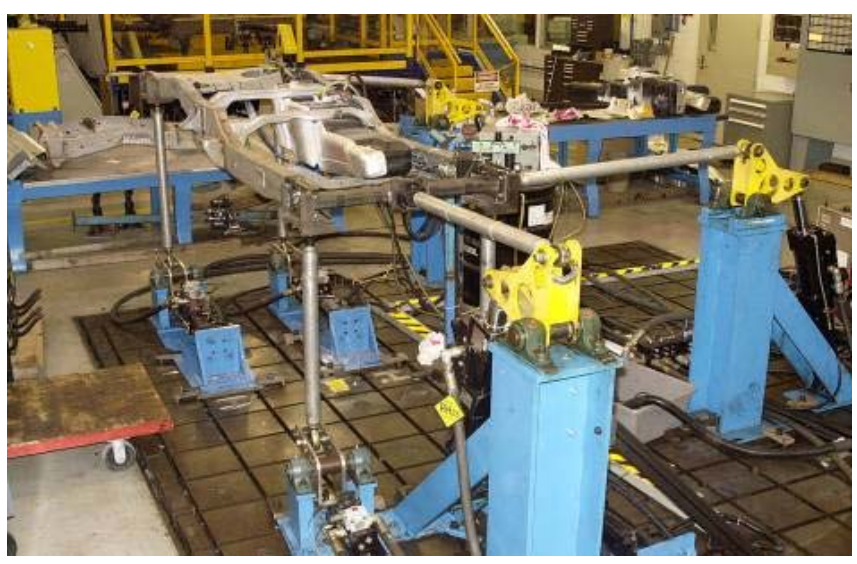

Figure 5-2 Fuel tank strap study setup in test (Photo Courtesy of [36]) 


\subsection{CAE Model Set up}

Figure 5-3 shows the CAE model in both nonlinear common mass method and nonlinear ALE method, which are developed with the same configuration and dimension as the test fixtures. Each component is represented by single color. In Figure 5-3 (a), the fuel tank is one component with equivalent mass to fuel tank and fuel total mass through adjusting the fuel tank density, while the separation between tow components, the fuel and fuel vapor, can be seen clearly in Figure 5-3 (b).

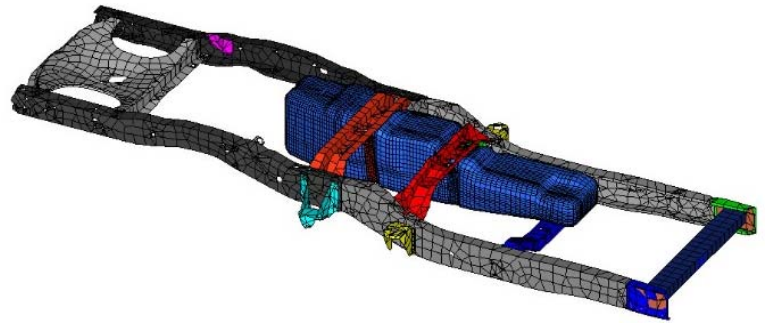

Figure 5-3 (a) CAE setup in Nonlinear Common Mass Method

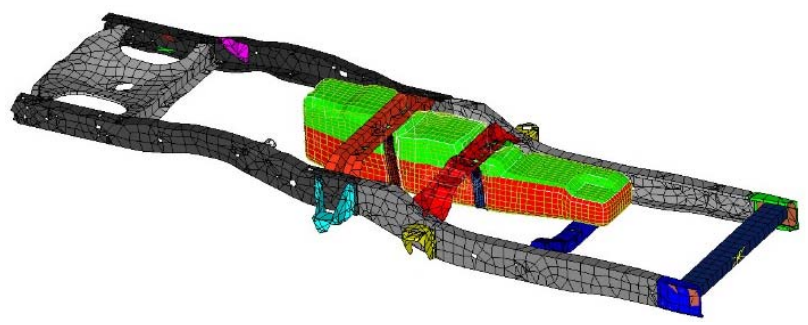

Figure 5-3 (b) CAE setup in Nonlinear ALE Method

5.3.1 Material property for fuel tank strap and fuel tank

The material properties for fuel tank strap and fuel tank are listed in Table 5-1.

Table 5-1 Material properties for fuel tank strap and fuel tank

\begin{tabular}{|c|c|c|c|}
\hline & Density (ton $\left./ \mathrm{mm}^{3}\right)$ & Young's Modulus (MPa) & Poisson's Ratio \\
\hline Steel & $7.87 \mathrm{e}-9$ & $2.07 \mathrm{e}+5$ & 0.3 \\
\hline $\begin{array}{l}\text { High-density } \\
\text { polyethylene }\end{array}$ & $9.3 \mathrm{e}-10$ & $2.0 \mathrm{e}+3$ & 0.5 \\
\hline
\end{tabular}


Figure 5-4 describes plastic true stress strain curve for fuel tank strap, and Figure 5-5 is plastic true stress strain curve for fuel tank.

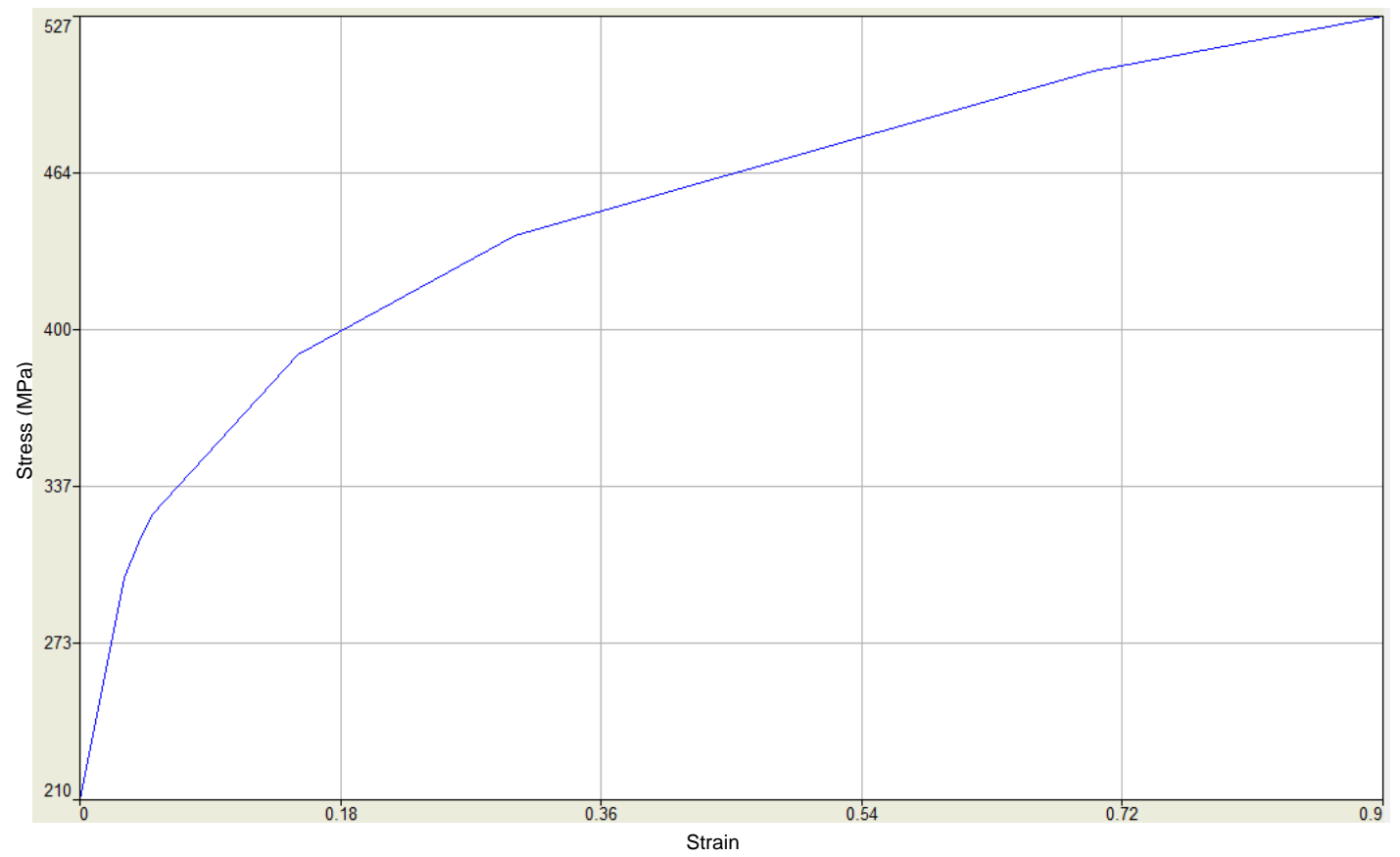

Figure 5-4 Fuel tank strap plastic stress strain curve

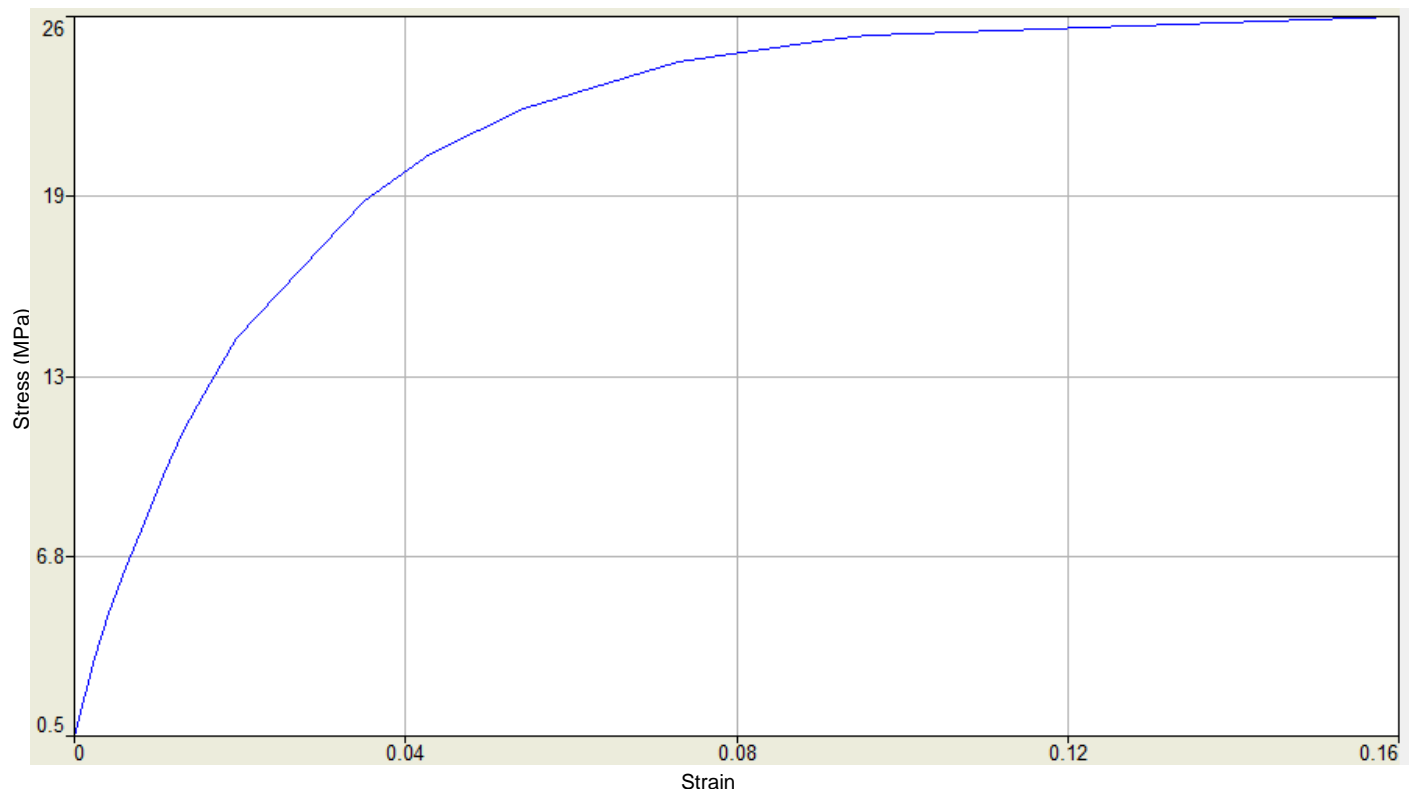

Figure 5-5 Fuel tank plastic stress strain curve 


\subsubsection{Modeling of Joints between Hydraulic Actuator and Frame}

To simulate the physical constraints in the lab, joints between hydraulic actuators and the frame are modeled with zero-length spring elements. To simulate a ball joint as shown in Figure $5-4$, very stiff springs in three translational D.O.F. and zero-stiffness springs in three rotational D.O.F. are used.

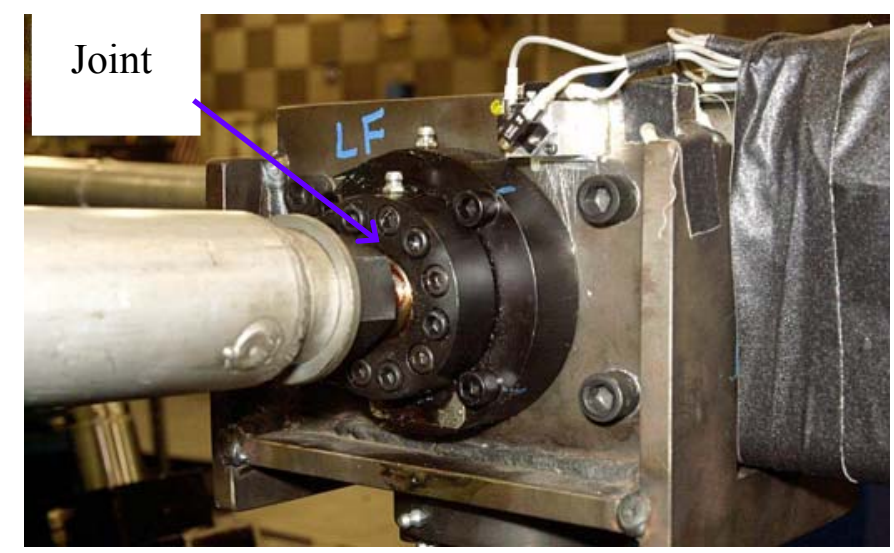

Figure 5-6 Joint between hydraulic actuator and frame in test (Photo Courtesy of [36])

\subsubsection{Modeling of the Frame}

To improve computation efficiency, a coarsened frame FE model, having the same first level natural frequencies, is derived from a baseline fine model, using an in-house modeling code. The same natural frequencies means the stiffness and mass in the coarsened model are still same as base model.

\subsubsection{Contact Surface Defining}

Contact surfaces are defined in the area between the fuel tank and upper and lower cross members, and in the area between the fuel tank and straps. To account for the differences of the elastic stiffness for plastics and steel, symmetry contact surfaces are defined between the fuel tank and straps with a scale factor for interface stiffness differences. At the outboard ends of the fuel 
tank straps are T-bars which are modeled as rigid bodies. Contact surfaces between cross members and T-Bars, and between strap inboard ends and the strap reinforcements are also defined. Contact surfaces between the fuel tank, fuel, and fuel vapor, can be defined through contact cards or coincident nodes.

\subsubsection{Road Load Input}

The table 5-2 lists the general proving ground route used to obtain vehicle structure durability and reliability data.

Table 5-2 Proving Ground Route Event

\begin{tabular}{|c|c|}
\hline & Route Event \\
\hline 1 & Suburban Driving Loop \\
\hline 2 & Power Hop Hill \\
\hline 3 & Silver Creek Hard Routes \\
\hline 4 & Acceleration Durability Road \\
\hline 5 & Access Roads \\
\hline 6 & Salt Bath \\
\hline 7 & Twist Ditch \\
\hline 8 & Simulated City Traffic Loop \\
\hline 9 & High Speed Track \\
\hline 10 & Dirt Cooling Loop \\
\hline 11 & $30 \%$ Grade/Reverse Brake \\
\hline 12 & Steering Cycles \\
\hline
\end{tabular}

The acceleration load inputs are collected from proving ground road test and processed to lab test load inputs, with considering time cycle and technician shift, to meet lab testing procedure for sub-system, like frame with fuel tank installed. Because the nonlinear explicit dynamic code is applied in simulation, only the most severe load and most critical load period is selected to highlight the critical hot spots and crack sequence to the greatest extent for durability failure, due to CPU time limitation. Therefore the nonlinear CAE simulation is the approximation to the testing, 
and the reasonable conclusion should be based on back to back comparison. Figure 5-5 lists the most severe road load in this testing with total seven load curves, four vertical load input, two lateral load input, one longitudinal load input. After comparing the amplitude in the whole time history, to reduce CPU time, only 12 seconds between 20 s and 40 s is selected as the most severe period.

Due to the solver is dynamic explicit code and its initial condition in Equation (5-6), the external input should be displacement. The test run has been made that when first time apply the acceleration load curves, the model is rollover, because the constant are different in seven load input when acceleration is integrated to displacement. So Matlab is applied to integrate acceleration directly to get displacement with filter to reduce the points in the time history.

Figure 5-6 shows a typical sample of displacement load input trimmed from the measured time history data. If a comparatively flat curve is added at the beginning of the sample displacement history curve, the displacements slowly ramp up to the first peak, resulting in more realistic damage. 
Left Frame Vert Accel @ trans X-member $(\mathrm{KF}=0.639)$ - Values

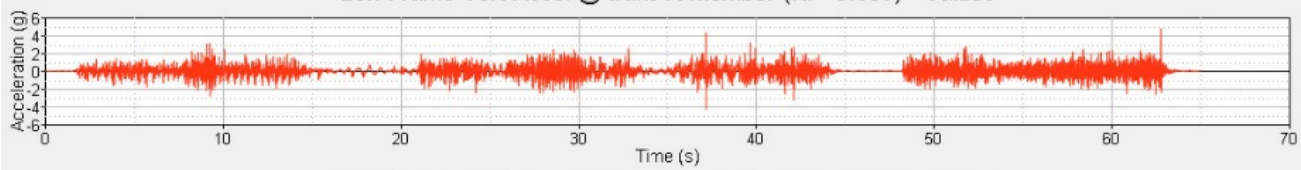

Right Frame Vert Accel @ trans X-member $(\mathrm{KF}=0.794)$ - Values

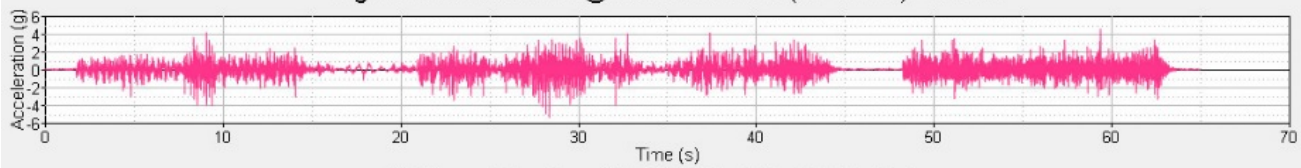

LR Frame Vert Accel @ shkl brkt $(K F=0.643)$ - Values

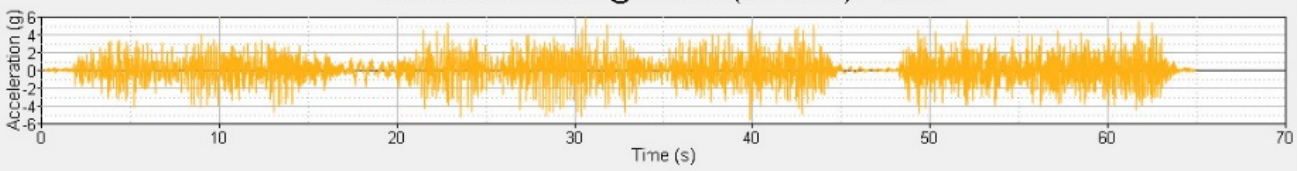

RR Frame Vert Accel @ shkl brkt (KF=0.958) - Values

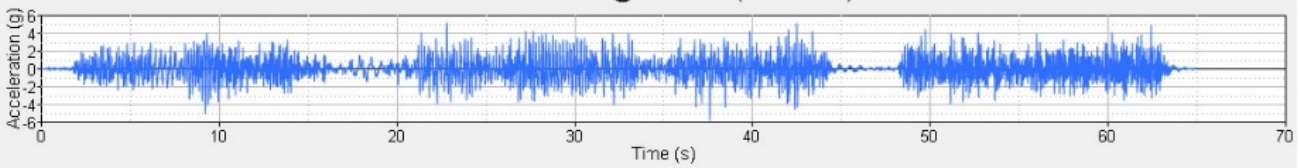

Left Frame Lat Accel @ trans X-member $(K F=0.892)$ - Values

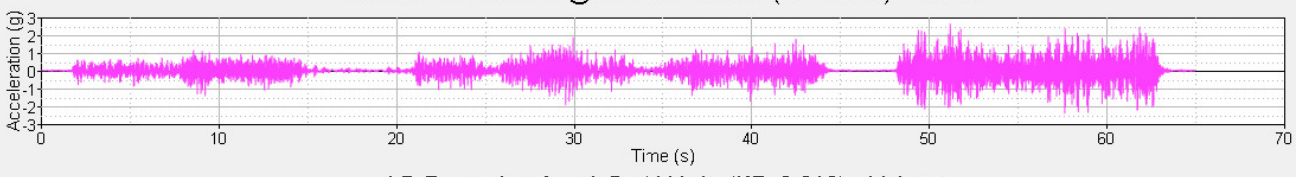

LR Frame Lat Accel @ shkl brkt $(K F=0.810)$ - Values

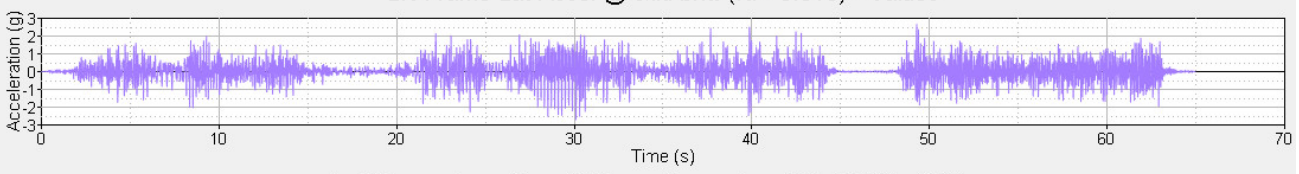

Left Frame Long Accel @ trans X-member $(\mathrm{KF}=0.921)$ - Values

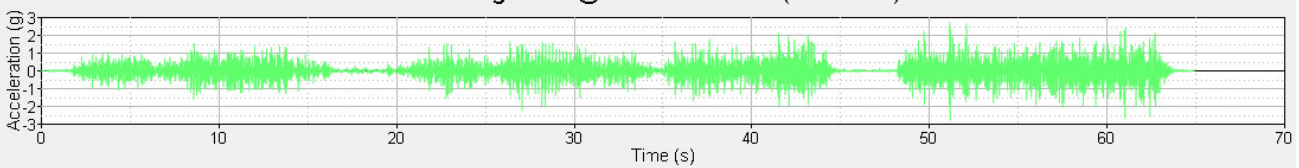

Figure 5-7 Acceleration load input in testing 


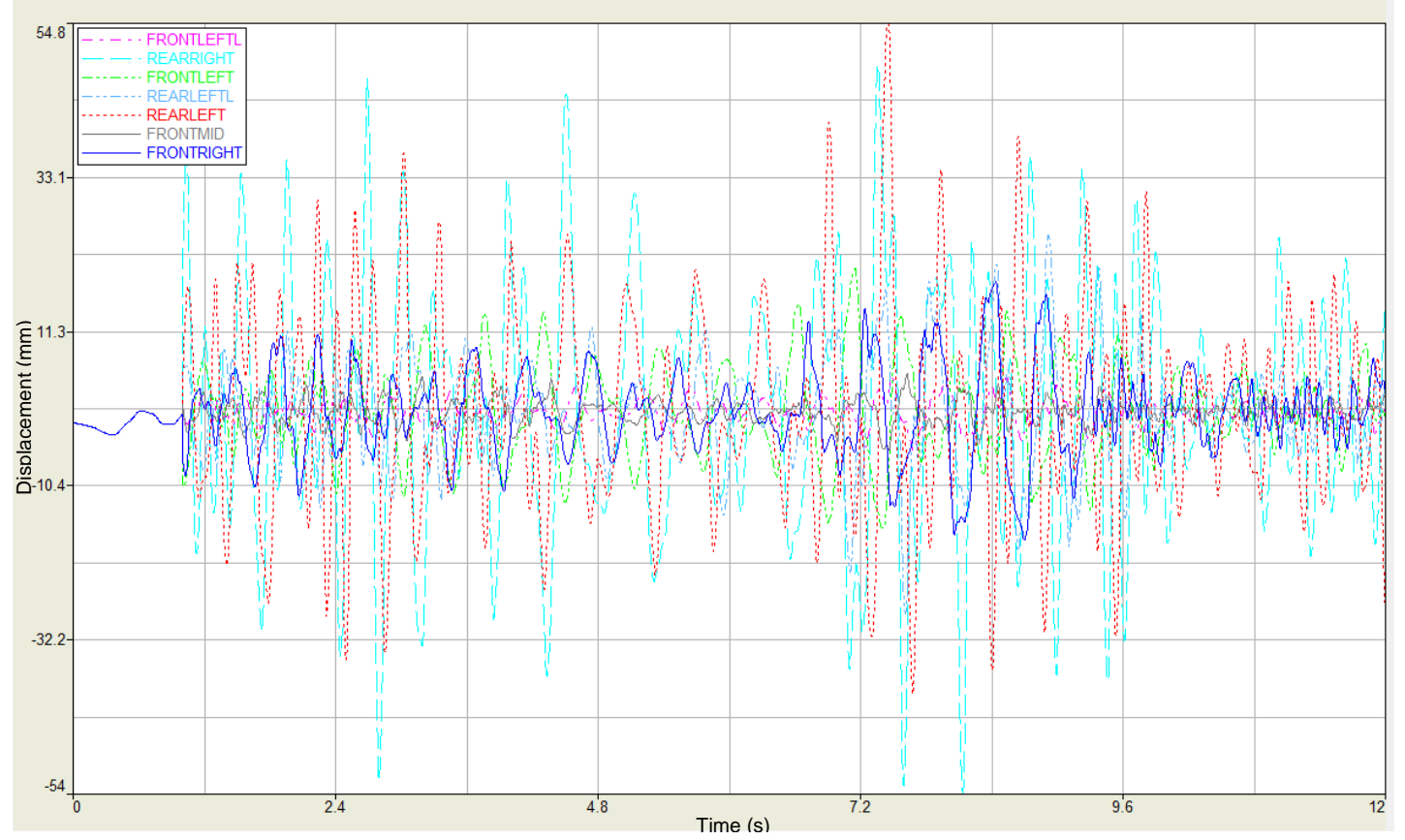

Figure 5-8 Filtered seven channel road load displacement input

\subsection{Experiment Results}

Strap samples were periodically inspected during the life cycle test and after test completion.

\subsubsection{Front Strap}

In the front strap, cracks were observed to initiate from the top upper spot weld in the Tend area. Additional cracks were observed on the front outboard edge. After the strap was removed, cracks were observed in the spot welds at the bolt end portion of the inboard side,

\subsubsection{Rear Strap}

In the rear strap, cracks were observed to initiate from the front outboard edge, and then from aft outboard edge. After the rear strap was removed, cracks were observed in the spot welds at the bolt end portion of the inboard side and in the inner face of the outboard side. 
Comparing the front and rear strap, the cracks at the leading edge of outboard side have similar initiation timing.

On the rear fuel tank strap, four tiny cracks were observed on front outboard edge and three on the aft outboard edge at the timing of a peak of load, as seen in Figure 5-10.

\subsection{CAE and Experiment Results Comparison}

Three coarsened FE models with the same sample displacement input were separately analyzed by MSC Nastran and RADIOSS with the full time gravity history as a boundary condition.

\subsubsection{Stress Analysis Results Comparison for Rear Strap}

1. Linear Fluid Volume Properties Definition Method

The fuel tank, with three fourths capacity defined by a property card, is used in Modal Transient Analysis. Contact surfaces among fuel tank, strap, and cross members are defined by rigid elements. Static "residual attachment modes" are employed in the analysis [23]. All material properties are linear. The displacement time history load input, the gravity card, and the damping coefficient are the same as those in the nonlinear methods. Maximum Von Mises stress results in Figure 5-11 show stress levels 15 times greater than those of the nonlinear methods - too great a difference from test results. These high stress levels indicated the rigid element contact surface were not accurate, and that fatigue analysis would be fruitless.

2. Common Mass Nonlinear Method

In post processing, displacement transient animation of the frame and fuel tank is observed, and is reasonable. The Von Mises stress pattern for the rear strap is shown in Figure 5-12. Compared with the test result, Figure 5-12 is approximately close. 


\section{ALE Nonlinear Method}

3-D Hexahedral elements are modeled to represent fuel and fuel vapor in the tank. During post processing, fuel sloshing can be seen through the animation with density distribution shown in Figure 5-9 at 5 time moments: $\mathrm{t}=0 \mathrm{~s}, \mathrm{t}=3 \mathrm{~s}, \mathrm{t}=6 \mathrm{~s}, \mathrm{t}=9 \mathrm{~s}, \mathrm{t}=12 \mathrm{~s}$.
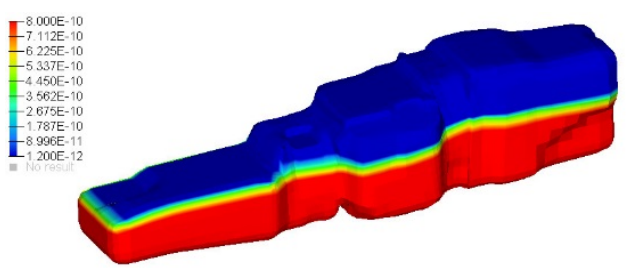

Figure 5-9 (a) Fuel sloshing in the fuel tank at 0 second

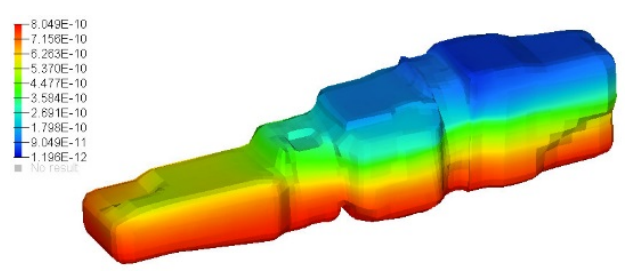

Figure 5-9 (c) Fuel sloshing in the fuel tank at 6 seconds

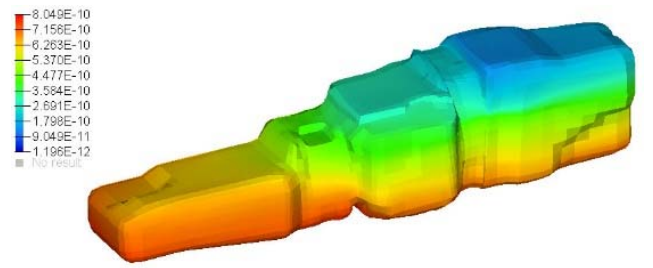

Figure 5-9 (e). Fuel sloshing in the fuel tank at 12 seconds

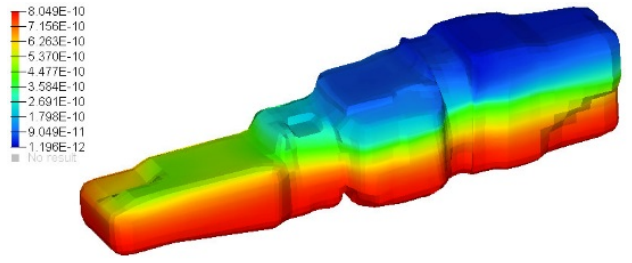

Figure 5-9 (b) Fuel sloshing in the fuel tank at 3 seconds

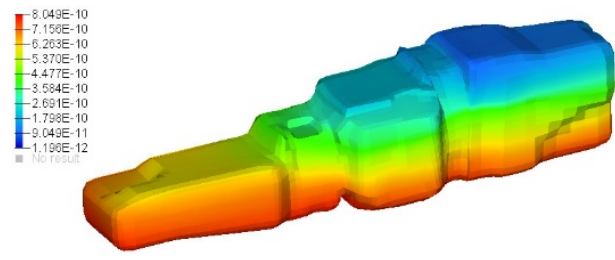

Figure 5-9 (d). Fuel sloshing in the fuel tank at 9 seconds

The Von Mises stress pattern for the rear strap is shown in Figure 5-13 with the same contour level setup, and with the same timing as in Figure 5-12. Compared with the test result shown in Figure 5-10, Figure 5-13 is the closest among the three CAE methods. 


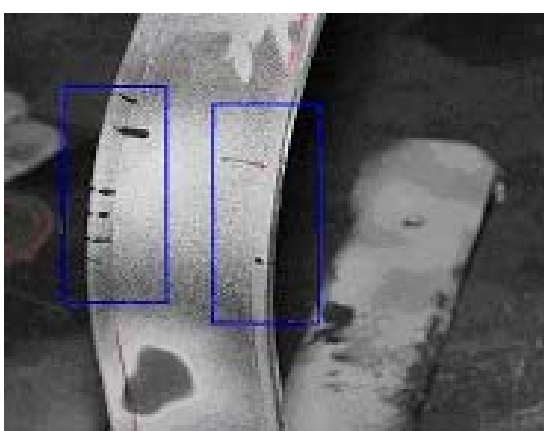

Figure 5-10 Cracks in outboard leading edge of rear strap (Photo Courtesy of [36])
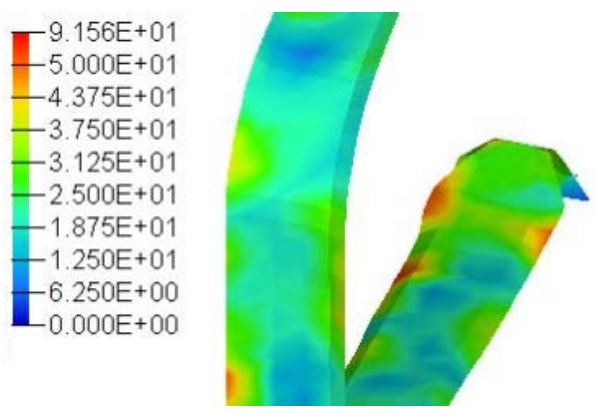

Figure 5-12 Von Mises stress in rear strap in Common Mass Nonlinear method
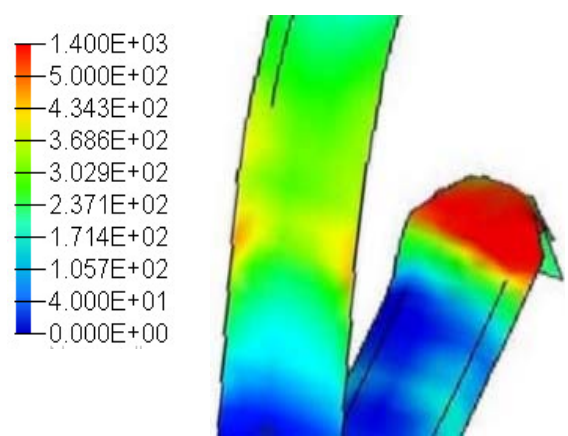

Figure 5-11 Von Mises stress in rear strap in Linear Fluid Volume Properties Definition method
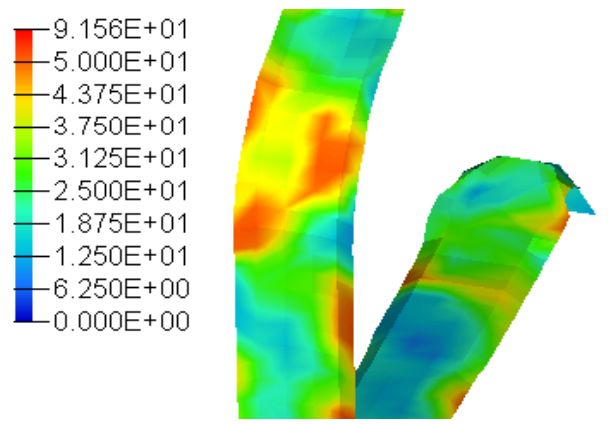

Figure 5-13 Von Mises stress in rear strap in ALE Nonlinear method

Plastic strain contour patterns for the two nonlinear methods are shown in Figure 5-14, 515 , at the same time for crack evaluation. It seems that both methods generate similar plastic strain pattern for rear strap. 


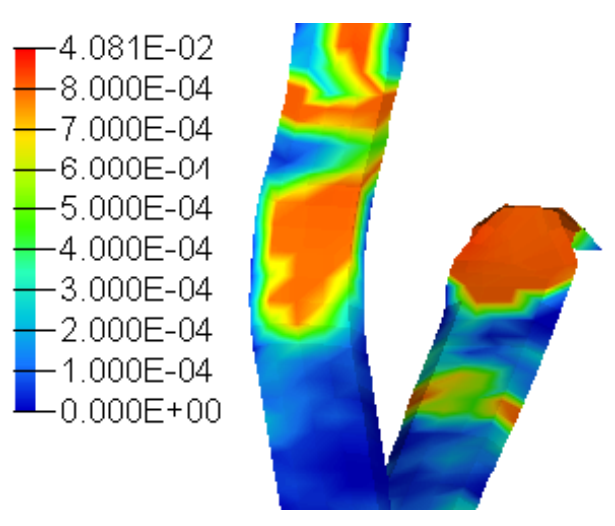

Figure 5-14 Plastics strain contour in rear strap in Common Mass Nonlinear method

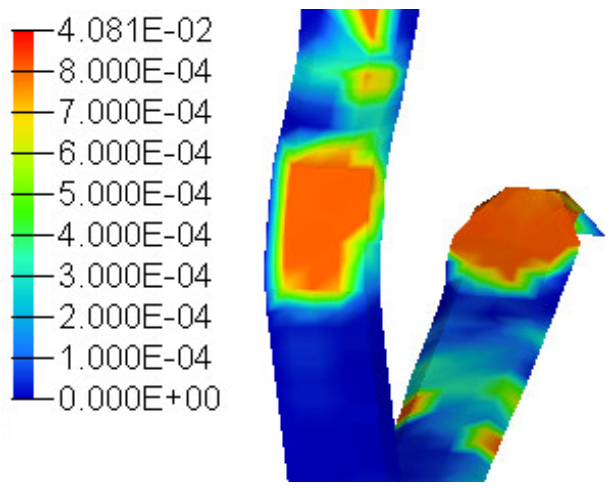

Figure 5-15 Plastics strain contour in rear strap in ALE Nonlinear method

So overall rear strap stress strain comparison show the nonlinear methods, common mass method and ALE method, are much more reasonable than Linear Fluid Volume Properties Definition Method.

\subsubsection{Stress Analysis Results Comparison between Font and Rear Strap}

Von Mises stress contour patterns between two straps in the three methods are shown in Figure 5-16, 5-17, 5-18 at the same time. Results show the linear method is too different from nonlinear methods and test that front strap stress is far less than rear strap. This is against the testing result that cracks have similar initiation timing on front and rear strap. So linear method can't predict reasonable result. 


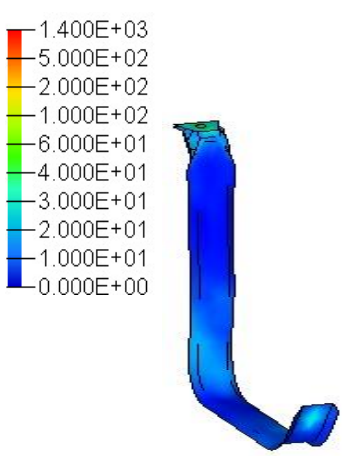

Figure 5-16 Von Mises stress contour in Left-to-Right side view in Linear Fluid Volume Properties Definition method

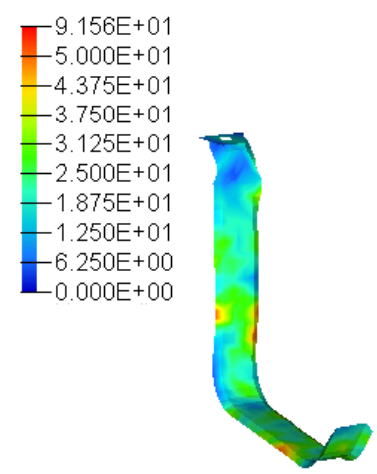

Figure 5-18 Von Mises stress contour in Left-to-Right side view in ALE Nonlinear method
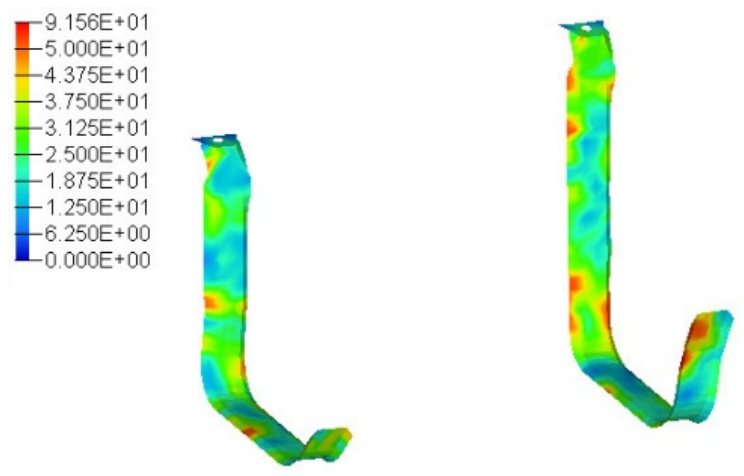

Figure 5-17 Von Mises stress contour in Left-to-Right side view in Common Mass Nonlinear method

Plastic strain contour patterns for the two nonlinear methods are shown in Figure 5-19, 520, at the same time for crack evaluation. Animation of the whole process for the two nonlinear methods, as shown in Figure 5-19 and 5-20, indicate the general trend of correct crack initiation between the two straps compared with test results. The first level cracks are initiated from welds of T-bar and inboard bolts. The second level cracks are initiated from inboard middle area. The third level cracks are initiated from both inboard and outboard corners. But in nonlinear common mass method relative higher strain shows on front strap which means cracks are more likely 
initiated from front strap earlier than rear strap and is against the testing result that cracks have similar initiation timing on front and rear strap. This conclusion will be further validated in fatigue analysis. 

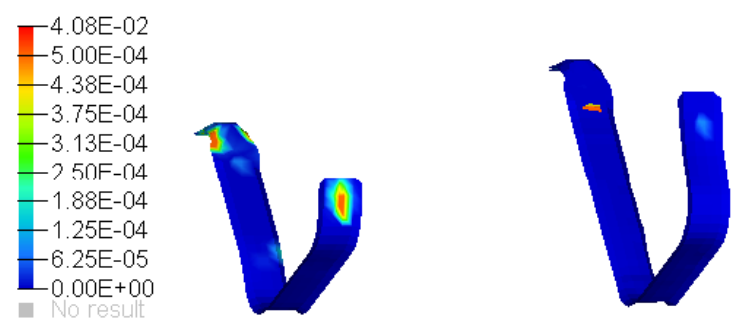

Figure 5-19 (a) Plastic Strain at 1 second in Common Mass Nonlinear method
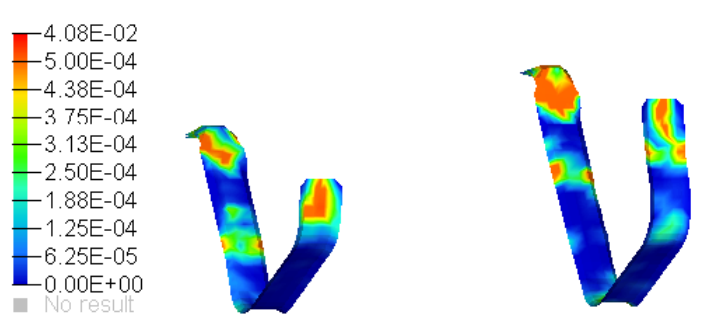

Figure 5-19 (b) Plastic Strain at 3 seconds in Common Mass Nonlinear method
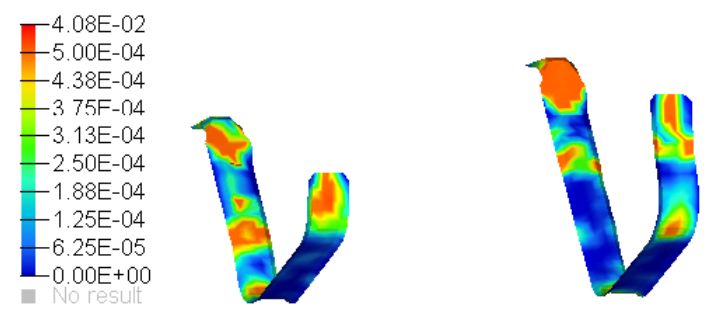

Figure 5-19 (c) Plastic Strain at 9 seconds in Common Mass Nonlinear method
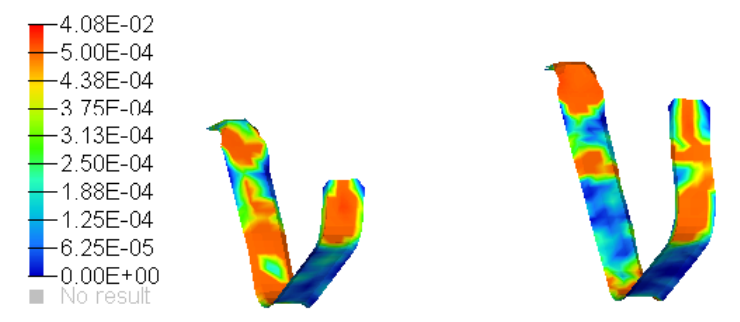

Figure 5-19 (d) Plastic Strain at 12 seconds in Common Mass Nonlinear method

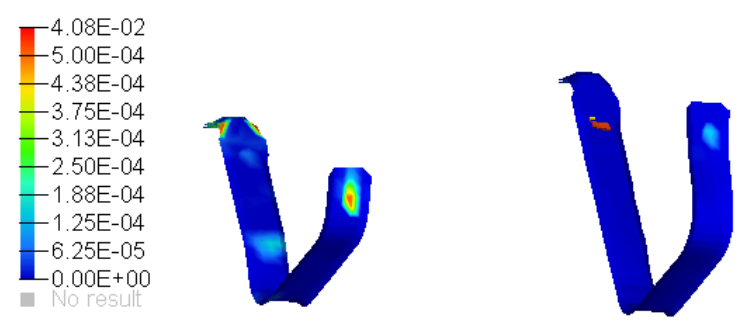

Figure 5-20 (a) Plastic Strain at 1 second in ALE Nonlinear method
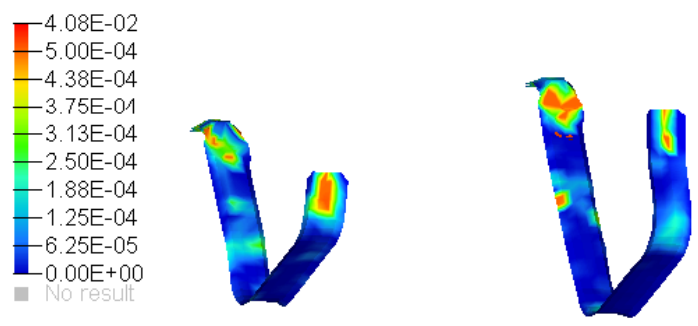

Figure 5-20 (b) Plastic Strain at 3 seconds in ALE Nonlinear method
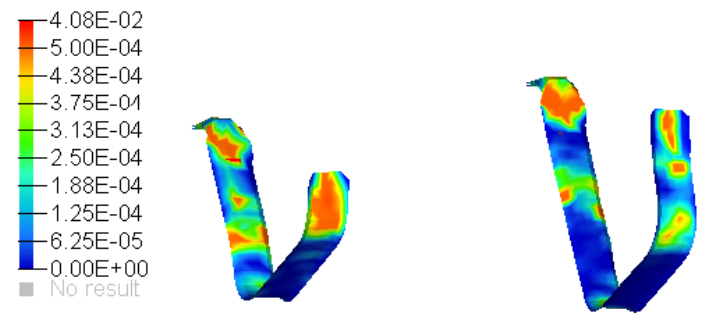

Figure 5-20 (c) Plastic Strain at 9 seconds in ALE Nonlinear method
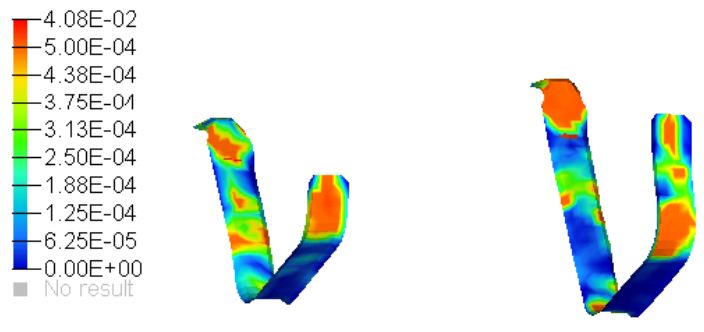

Figure 5-20 (d) Plastic Strain at 12 seconds in Common Mass Nonlinear method 


\subsubsection{Fatigue Analysis Result}

Upper and lower surface membrane strains are retrieved from the result output files and compared at each time step. The worse surface membrane strain is selected and therefore a strain time history is formed. Then the format is changed to meet nCode DesignLife input requirements, through a user program in FORTRAN. Table 5-3 lists the fatigue property of steel applied in fatigue calculation. Comparing the fatigue life contour patterns (Figure 5-21 and 5-22, at the same fatigue life scale), ALE method, Figure 5-22, correlates very closely in both crack initiation time, sequence, and location with test results that cracks has similar initiation time on both front and rear strap, while the nonlinear common mass method shows too less fatigue life on front strap. And this further validates the conclusion from stress strain comparison between front and rear strap.

Table 5-3 Fatigue property of steel

\begin{tabular}{|c|c|c|}
\hline YS & 250 & Yield Strength (MPa) \\
\hline UTS & 354 & Ultimate Tensile Strength (MPa) \\
\hline $\mathrm{E}$ & 2.07E5 & Elastic Modulus (MPa) \\
\hline me & & Elastic Poisson's Ratio \\
\hline $\mathrm{mp}$ & & Plastic Poisson's Ratio \\
\hline Sf' & 782 & Fatigue Strength Coefficient (MPa) \\
\hline b & -0.11 & Fatigue Strength Exponent \\
\hline c & -0.41 & Fatigue Ductility Exponent \\
\hline Ef' & 0.19 & Fatigue Ductility Coefficient \\
\hline$n^{\prime}$ & 0.27 & Cyclic Strain Hardening Exponent \\
\hline$n^{\prime} 90$ & & Out of phase Cyclic Strain Hardening Exponent \\
\hline$K^{\prime}$ & 1259 & Cyclic Strength Coefficient (MPa) \\
\hline$K^{\prime} 90$ & & Out of phase Cyclic Strength Coefficient (MPa) \\
\hline Nc & $2 \mathrm{E} 8$ & Cut-off (Reversals) \\
\hline SEe & 0 & Standard Error of Log(e) (Elastic) \\
\hline SEp & 0 & Standard Error of Log(e) (Plastic) \\
\hline SEC & 0 & Standard Error of Log(e) (Cyclic) \\
\hline $\mathrm{Ne}$ & & Endurance Limit (Reversals) \\
\hline FSN & 0.6 & Fatemi-Socie parameter \\
\hline $\mathrm{S}$ & 1 & Wang Brown parameter \\
\hline
\end{tabular}



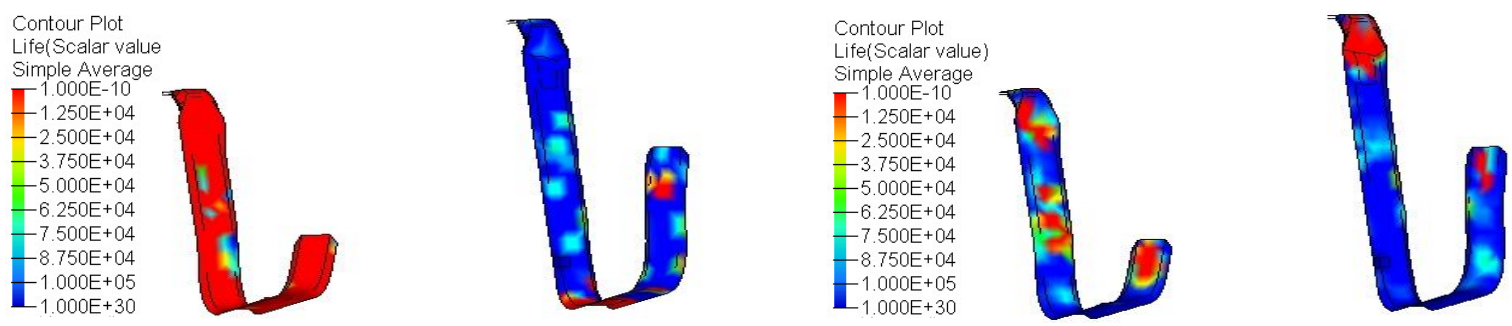

Figure 5-21 Fatigue life contour in Leftto-Right side view in Common Mass

Nonlinear method

Figure 5-22 Fatigue life contour in Leftto-Right side view in ALE Nonlinear method

\subsection{CPU Time Comparison}

Table 5-4 lists CPU time comparison for three methods, which show linear method's advantage in CPU time, therefore it is more critical for modeling the coarse model and control the total number of solid elements defined by ALE method to keep CPU time as less as possible. But in consideration that nowadays computer is so powerful, CPU time is already not a major factor to concern for regular job.

Table 5-4 CPU time comparison

\begin{tabular}{|c|c|}
\hline Method & CPU Time \\
\hline Linear Fluid Volume Properties Definition Method & 0.5 hours \\
\hline Common Mass Nonlinear Method & 2.5 hours \\
\hline ALE Nonlinear Method & 5 hours \\
\hline
\end{tabular}

\subsection{Conclusion}

Three methods are compared in stress, plastic strain, and fatigue life, respectively. The first comparison in stress clearly shows that Linear Fluid Volume Properties Definition method gives too high stress level and wrong pattern from testing. The second comparison in plastic strain 
exhibits that ALE nonlinear method can correlate correct cracks initiation sequence and locations between front and rear strap, while Common Mass nonlinear method shows much worse cracks appeared in front strap than rear strap which is against the testing. The third comparison in fatigue life further validates that ALE nonlinear method correlates very closely in both crack initiation sequence and location with test results that cracks has similar initiation time on both front and rear strap, while the nonlinear common mass method shows too less fatigue life on front strap. Overall, The ALE nonlinear dynamic method is more realistic in theory for simulating fuel tank movement with fuel sloshing, and is better to predict the sequence and location of crack initiation. 


\section{CHAPTER 6 CONCLUSION}

The Arbitrary Lagrangian-Eulerian (ALE) is a hybrid finite element formulation that can alleviate many of the drawbacks from the traditional Lagrangian-based and Eulerian-based finite element simulations, which is developed through combining modern algorithms for Lagrangian hydrodynamics, meshing technology and remap methods developed for high-resolution Eulerian methods. When using the ALE technique in engineering simulations, the computational mesh inside the domains can move arbitrarily to optimize the shapes of elements, while the mesh on the boundaries and interfaces of the domains can move along with materials to precisely track the boundaries and interfaces of a multi-material system. The use of Arbitrary Lagrangian-Eulerian (ALE) computer codes has been an enabling technology for many important applications, such as Forming Processes, Two-Phase Flows with Phase Change and Heat Transfer of Computer Chips, and Plasma Physics, etc. When using the ALE technique in engineering simulations, the computational mesh inside the domains can move arbitrarily to optimize the shapes of elements, while the mesh on the boundaries and interfaces of the domains can move along with materials to precisely track the boundaries and interfaces of a multi-material system.

In automotive CAE analysis, simulation of fuel tank potential leakage under impact in crashworthiness and dynamic stress and fatigue life of fuel tank straps under proving ground condition in durability are active problems for vehicle design. Typically a fuel tank is held with fuel tank straps. Its movement lies in the domain of nonlinear large rotation dynamics. Moreover, the sloshing behavior in the fuel tank makes the problem even more intricate. ALE method is being applied in fuel tank leakage safety simulation with vehicle at high speed impact, while this ALE method application is relatively too easy because the load input is too simple, an initial velocity, that won't cause too much CPU time to be used. In current fuel tank strap simulation, one available 
method that directly applies the proving ground load specifically requested for fuel tank strap is not included in the standard testing procedure for full vehicle testing, because load cell installation is very complex and will induce problems which means it is not practical. The second method is fluid volume properties definition method to give a fuel material property to generate a virtual mass for the volume of the incompressible fuel. This method assumes that the free surface has zero pressure with no sloshing effects and no viscous (rotational flow) effects exist, so it can give satisfactory results in small displacement environments, but cannot predict the structural responses in large rotation situations.

In order to improve the weakness of above two methods, the research here successfully applies ALE method in fuel tank strap simulation under proving ground condition and further fatigue calculation. Through comparing existing methods applied in fuel tank and fuel tank strap CAE application, the research here did make some breakthrough in below areas.

1) Apply ALE method to simulate fuel sloshing and integrate with proving ground time history load.

The ALE has been applied in safety CAE to study fuel sloshing impact to the fuel tank in different volume under initial velocity which is a too simple load case that won't take too long CPU to finish run, although it is an innovation for fuel tank modeling. But the method to integrate ALE method for fuel sloshing and the proving ground time history load for the fuel tank strap durability simulation is first time in auto industry. Through comparing three different methods and testing, results show that fuel sloshing did play a critical role to affect the fuel mass redistribution and the stress variation with time, therefore prove the critical contribution from ALE method. 
2) Set up procedure how to simplify CAE model to reduce CPU time

The analysis with durability proving ground time history load input will cost much more CPU time than normal initial velocity load input. So how to reduce CPU time, while still keep the accuracy, is becoming top priority to control analysis within reasonable CPU time limit. This procedure figures out several ways in modeling, a) coarse area away from fuel tank and fuel tank strap, but must keep the new coarsened model the same frequencies as original model, which means mass and stiffness is still same and new coarsened model is correct. The fuel tank and strap are the target of analysis and still modeled in detail, so that it will achieve accurate stress strain result. b) use common nodes to define the contact between fuel, fuel vapor, and fuel tank. The contact surface computation is usually the large portion of CPU time. In this research, the contact between fuel tank and fuel tank strap, fuel tank and frame, fuel tank strap and frame are critical areas which should be kept. This common nodes modeling method won't cause any issue, but reduce CPU time greatly. With all the above modeling skill, the overall CPU time for ALE method is limited to five hours which is pretty efficient and will be even more efficient with the more powerful computer developed.

3) Simplify and process the testing load in MATLAB to reduce CPU time and avoid the numerical issue in explicit dynamic transient code

As the initial condition of dynamic explicit code, the input should be displacement. But the testing acquired data is acceleration time history. As a test run to show, the model will be immediately rollover with seven acceleration time history input, due to the different constants generated from integration of those acceleration time history input. Therefore a new MATLAB code is programmed to integrate acceleration to displacement in exactly 
same constant for those seven acceleration time history. Zero padding the data is applied before the DFT to increase the frequency resolution and thus improve the amplitude estimate. To avoid the spectrum leakage, Hanning windows is applied in signal processing as moderate window. The CAE result animation shows the realistic movement of testing fixture.

4) Interface with fatigue code through programing to post process the intermediate results files.

The elastic-plastics method with uniaxial strain life fatigue theory is used in this analysis. First, the total strain life time history from the RADIOSS stress analysis results is input to the program in FORTRAN. The top membrane strain and bottom membrane strain are compared according to strain rate, then the higher strain rate history are composed. Second, the material cyclic stress strain curve is used to track stress strain history, and strain cycles and the mean stress are output. Third, the Smith-Watson-Topper equation is used to get damage from each strain cycle.

Future work will be focused on how to improve the fuel solid modeling. For this study, in order to achieve more accurate result, Hexahedral element type is used for fuel solid modeling in ANSYS ICEM CFD, a popular propriety software package used for CAD and mesh generation. Relatively long time is spent due to fuel tank geometry complexity. So a more efficient solid modeling method and software are needed for future work. 


\section{REFERENCES}

1. Hirt, C. W., Amsden, A. A., and Cook, J. L., “An Arbitrary Lagrangian-Eulerian computing method for all flow speeds.” Journal of Computational Physics, Vol. 14, No. 3, 1974, pp. 227 $-53$.

2. Boris, J. P. and Book, D. L., "Flux-Corrected Transport I. SHASTA, A Fluid Transport Algorithm that Works,” Journal of Computational Physics, Vol. 11, 1973, pp. 38-69.

3. van Leer, B., "Towards the Ultimate Conservative Difference Scheme. IV. A New Approach to Numerical Convection,” Journal of Computational Physics, Vol. 23, 1977, pp. 276-299.

4. Caramana, E. J., Burton, D. E., Shashkov, M. J., and Whalen, P. P., "The Construction of Compatible Hydrodynamics Algorithms Utilizing Conservation of Total Energy,” Journal of Computational Physics, Vol. 146, 1998, pp. 227-262.

5. Caramana, E. J., Shashkov, M. J., and Whalen, P. P., "Formulations of Artificial Viscosity for Multi-Dimensional Shock Wave Computations,” Journal of Computational Physics, Vol. 144, 1998, pp. 70-97.

6. Caramana, E. J., Rousculp, C. L., and Burton, D. E., “A compatible, energy and symmetry preserving Lagrangian hydrodynamics algorithm in three-dimensional cartesian geometry.” Journal of Computational Physics, Vol. 157, No. 1, 2000, pp. 89-119.

7. Caramana, E. J. and Whalen, P. P., "Numerical preservation of symmetry properties of continuum problems.” Journal of Computational Physics, Vol. 141, No. 2, 1998, pp. 174-98.

8. Loubere, R. and Caramana, E. J., "The force/work differencing of exceptional points in the discrete, compatible formulation of Lagrangian hydrodynamics.” Journal of Computational Physics, Vol. 216, No. 1, 2006, pp. 1-18. 
9. Bauer, A. L., Burton, D. E., Caramana, E. J., Loubere, R., Shashkov, M. J., and Whalen, P. P., “The internal consistency, stability, and accuracy of the discrete, compatible formulation of Lagrangian hydrodynamics.” Journal of Computational Physics, Vol. 218, No. 2, 2006, pp. 572-93.

10. Benson, D. J., “A new two-dimensional flux-limited shock viscosity for impact calculations,” Computer Methods in Applied Mechanics and Engineering, Vol. 93, No. 1, December 1991, pp. 1991.

11. Benson, D. J., “Computational Methods in Lagrangian and Eulerian Hydrocodes,” Computer Methods in Applied Mechanics and Engineering, Vol. 99, 1992, pp. 235-394.

12. Schulz, W. D., “Tensor artificial viscosity for numerical hydrodynamics," Journal of Mathematical Physics, Vol. 5, No. 1, January 1964, pp. 133-138.

13. Wilkins, M. L., “Use of Artificial Viscosity in Multidimensional Fluid Dynamic Calculations,” Journal of Computational Physics, Vol. 36, 1980, pp. 281-303.

14. Noh, W. F., "Errors for calculations of strong shocks using an artificial viscosity and an artificial heat flux,” Journal of Computational Physics, Vol. 72, No. 1, September 1987, pp. 78-120.

15. Allen C. Robinson, Tomas A. bunner, Susan Carroll, Richard Drake, et al., “ALEGRA: Ann Arbitrary Lagrangian-Eulerian Multimaterial, Multiphysics Code”, 46 ${ }^{\text {th }}$ AIAA Aerospace Sciences Meeting and Exhibit, 7-10 January 2008, Reno, Nevada

16. Christiaan Stoker, "Developments of the Arbitrary Lagrangian-Eulerian Method in non-linear Solid Mechanics, Applications to Forming Processes”, PHD Thesis, 1999 
17. Gustavo Rabello Dos Anjos, “A 3D ALE Finite Element Method for Two-Phase Flows with Phase Change and heat transfer for interlayer cooling of the new generation of multi-stacked computer chips.”, PHD Thesis, 2012

18. Milan Kuchǎr’ı, “Arbitrary Lagrangian-Eulerian (ALE) Methods in Plasma Physics”, PHD Thesis, 2006

19. Nuno Miguel DINIZ DOS SANTOS, "Numerical methods for fluid-structure interaction problems with heart valves”, PHD Thesis, 2013

20. Tang, B., Guha, S., Tyan, T., Doong, J. et al., "Simulation of Sloshing and Ballooning in Fuel Tanks for High Speed Impacts,” SAE Technical Paper 2006-01-0314, 2006, doi:10.4271/2006$\underline{01-0314}$

21. Lin, B., Gundle, M., Rowley, M., Aloe, A. “Fuel Tank Strap Fatigue Sensitivity Study under Fuel Level Variation and Payload Variation”, SAE Technical Paper 2014-01-0921, 2014, doi:10.4271/2014-01-0921.

22. Qin, P. and D'Souza, S., “CAE Fatigue Prediction of Fuel Tank Straps using Proving Ground Loads,” SAE Technical Paper 2005-01-1405, 2005, doi:10.4271/2005-01-1405.

23. MSC Nastran 2014, Dynamic Analysis User’s Guide, Chapter 11:Coupled Fluid-Structure Analysis

24. MSC Nastran 2014, Dynamic Analysis User’s Guide, Chapter 5:Transient Response Analysis 25. https://en.wikipedia.org/wiki/Load_cell\#Strain_gauge_load_cell

26. Lars Olovsson, M’hamed Souli, “ALE and Fluid-Structure Interaction Capabilities in LSDYNA”, $6^{\text {th }}$ International LS-DYNA Conference

27. Radioss Theory Manual

28. John O. Hallquist, LS-DYNA Theory Manual 
29. http://www.dynaexamples.com/ale

30. Arnau Folch Duran, "A Numerical Formulation to solve the ALE Navier-Stokes Equations Applied to the Withdrawl of Magma Chambers”, PHD Thesis, 2000

31. "ALE - SPH LATERAL IMPACT ON A FUEL TANK", Edited by RADIOSS Consulting Corporation, September 2001

32. nCode DesignLife User Manual

33. http://www.dynasupport.com/tutorial/ls-dyna-users-guide/time-integration

34. Sando Han, Jin Wha Chung, "Retrieving Displacement Signal From Measured Acceleration Signal”

35. https://en.wikipedia.org/wiki/Window_function\#Windowing

36. Song, G., Shu, K., Khatib-Shahidi, B., Ourchane, A. et al., "Nonlinear Dynamic Simulation of Fuel Tank Strap Stress and Fatigue Life under Proving Ground Conditions," SAE Technical Paper 2005-01-0979, 2005, doi:10.4271/2005-01-0979

37. Digges, K., Fournier, E., Keown, M., Shewchenko, N. et al., “Alternative Fuel Tanks for Pickups with Sidesaddle Tanks,” SAE Technical Paper 2005-01-1427, 2005, doi:10.4271/2005-01-1427.

38. Craig, R., Qu, T., Pan, L., Tyan, T. et al., "Crash Performance Simulation of a Multilayer Thermoplastic Fuel Tank with Manufacturing and Assembly Consideration,” SAE Int. J. Mater. Manuf. 4(1):27-39, 2011, doi:10.4271/2011-01-0009.

39. Li, F., Sibal, S., and Ding, P., “An Integrated Fuel Tank System Simulation,” SAE Technical Paper 2011-01-0792, 2011, doi:10.4271/2011-01-0792.

40. Li, F., Sibal, S., McGann, I., and Hallez, R., “Radiated Fuel Tank Slosh Noise Simulation,” SAE Technical Paper 2011-01-0495, 2011, doi:10.4271/2011-01-0495. 
41. Griffith, J., Machado, C., and Bendele, B., “Comparative Evaluation of Automotive Fuel Tanks in General Accordance with ECE R34.01, Annex 5 Section 5.0 "Resistance to Fire”," SAE Technical Paper 2005-01-1561, 2005, doi:10.4271/2005-01-1561.

42. Lin, B., “A New Method of d'Alembert's Principle Finite Element Based Fatigue Calculation with Input of Loads and Accelerations,” SAE Technical Paper 2013-01-1003, 2013, doi: $\underline{10.4271 / 2013-01-1003 .}$.

43. Wang, Z. Rakheja, S., and Sun, C., "Influence of Partition Location on the Braking Performance of a Partially-Filled

44. Tank Truck,” SAE Technical Paper 952639, 1995, doi:10.4271/952639.

45. Kang, X., Rakheja, S. and Stiharu, I., “Directional Dynamics of a Partly-Filled Tank Vehicle under Braking and Steering,” SAE Technical Paper 2000-01-3477, 2000, doi:10.4271/200001-3477.

46. Dutta, S. and Laha, M. K., “Analysis of the small amplitude sloshing of a liquid in a rigid container of arbitrary

47. shape using a low-order boundary element method”, Int. J. Numer. Meth. Engng., 47:16331648, 2000.

48. Hayashi, K., Mikami, M., and Miura, K., “Development of Multi-Layer Plastic Membrane (Bladder Membrane) for Vapor Reducing Fuel Tank,” SAE Technical Paper 2001-01-1120, 2001. doi:10.4271/2001-01-1120.

49. Banerjee, R., Isaac, M., Oliver, L., and Breig, W., “A Numerical Study of Automotive Gas Tank Filler Pipe Two Phase Flow,” SAE Technical Paper 2001-01-0732, 2001, doi: 10.4271/2001-01-0732. 
50. Banerjee, R., Bai, X., Pugh, D., Isaac, K.M. et al., “CFD Simulations of Critical Components in Fueling Systems,” SAE Technical Paper 2002-01-0573, 2002, doi:10.4271/2002-01-0573.

51. Kamiya, K., Yamaguchi, Y., de Vries, E., “Simulation Studies of Sloshing in a Fuel Tank,” SAE Technical Paper 2002-01-0574, 2002, doi:10.4271/2002-01-0574.

52. IU, H.S, Cleghorn, W.L. and Mills, J. K., “Design and Analysis of Fuel Tank Baffles to Reduce the Noise Generated from Fuel Sloshing,” SAE Technical Paper 2004-01-0403, 2004, doi:10.4271/2004-01-0403.

53. Ma, J. and Usman, M. : "Modeling of Fuel Sloshing Phenomena Considering Solid-Fluid Interaction”, 8th International LS-DYNA User Conference

54. Roh, W-J., Cho, S.-H. and Park, J. I., "Simulation of Sloshing in Fuel Tanks and Parametric Study on Noise Reduction by Decreasing Impact Pressure,” SAE Technical Paper 2005-011913, 2005, doi:10.4271/2005-01-1913.

55. Kamel, M., Hanai, J., Fukasawa, W. and Makino, T., "Establishment of a Method for Predicting and Confirming Fuel Tank Sloshing Noise,” SAE Technical Paper2007-01-1538, 2007, doi:10.4271/2007-01-1538.

56. Himeki, H., Kumagai, H., and Morohoshi, K., "Fatigue Behavior Analysis and Durability Evaluation of Plastic Fuel Tank,” SAE Technical Paper 2006-01-0782, 2006, doi:10.4271/2006-01-0782.

57. van der Veen, W. A., “Crushing Simulation of a Partially Filled Fuel Tank Beyond Failure,” SAE Technical Paper 2005-01-1426, 2005, doi:10.4271/2005-01-1426.

58. Ding, P., Buijk, A. J., van der Veen, W. A., “Simulation of Fuel Tank Filling Using a Multimaterial Euler Solver with Multiple Adaptive Domains,” SAE Technical Paper 2005-01-1915, 2005, doi:10.4271/2005-01-1915. 
59. Tang, B.-R., Guha, S., Tyan, T., Doong, J., Shaner, L., and Bhalsod, D., "Simulation of Sloshing and Ballooning in Fuel Tanks for High Speed Impacts,” SAE Technical Paper 200601-0314, 2006, doi:10.4271/2006-01-0314.

60. Matteo, L. D., Fortunato, F. Oliva, P., Fiore, N., and Martinett, M., "Sloshing Analysis of an Automotive Fuel Tank,” SAE Technical paper 2006-01-1006, 2006, doi:10.4271/2006-011006.

61. Benrabah, Z., Thibault, F. and DiRaddo, R., "Finite Element Modeling of Fuel Emission for Thermoplastic Multilayer Fuel Tanks with Optimization of Barrier Properties,” SAE Technical Paper 2006-01-0625, 2006, doi:10.4271/2006-01-0625.

62. Chang, J., Tyan, T., El-bkaily, M., Marpu, A., Zhang, Q. and Santini, J., "Implicit and Explicit Finite Element Methods for Crash Safety Analysis,” SAE Technical Paper 2007-01-0982, 2007, doi:10.4271/2007-01-0982.

63. Craig, R., Chen, Y., Tyan, T., Laya, J. and Cheng, J., "Finite Element Modeling of the Frame for Body-on-Frame Vehicle, Part I - Subsystem Investigation,” SAE Technical Paper 200401-0688, 2004, doi:10.4271/2004-01-0688.

64. Chen, Y., Craig, R., Tyan, T., Laya, J. and Cheng, J., "Finite Element Modeling of the Frame for Body-on-Frame Vehicle, Part II - Full Vehicle Crash,” SAE Technical Paper 2004-010689, 2004, doi:10.4271/2004-01-0689.

65. Gao, R., Xi, C., Tyan, T., Mahadevan, K. and Doong, J., “A Practical Approach to Consider Forming Effects for Full Vehicle Crash Application,” SAE Technical Paper 2009-01-0471, 2009, doi:10.4271/2009-01-0471. 
66. Gao, R., Pan, L., Tyan, T., Mahadevan, K., Ghouati, O., Lanzerath, H. and Kessen, M., “Impact Simulation of Hydroformed Front End Vehicle Structure,” SAE Technical Paper 2006-010312, 2006, doi:10.4271/2006-01-0312.

67. Gonzalez Noble, M., Huang, M., Tyan, T., Shaner, L., Ghouati, O., Lanzerath, H., Wu, B. and Dombek, B., “Testing and Finite Element Modeling of Hydroform Frames in Crash Applications,” SAE Technical Paper 2007-01-0981, 2007, doi:10.4271/2007-01-0981.

68. Banerjee, R., Bai, X., Pugh, D., Isaac, K.M. et al, "CFD Simulations of Critical Components in Fueling Systems", SAE Paper 2002-01-0573.

69. Kamiya, K., Yamaguchi, Y., E. de Vries, "Simulation Studies of Sloshing in a Fuel Tank", SAE Paper 2002-01-0574

70. W. A. van der Veen, "Crushing Simulation of a Partially filled Fuel Tank Beyond Failure", SAE Paper 2005-01-1426

71. Won-Joo Roh, Sok-Hyun Cho and Jae In Park, "Simulation of Sloshing in Fuel Tanks and Parametric Study on Noise Reduction by Decreasing Impact Pressure", SAE 2005-01-193

72. IU, H.S, Cleghorn, W.L. and Mills, J.K.: “Design and Analysis of Fuel Tank Baffles to Reduce the Noise Generated from Fuel Sloshing”, SAE paper 2004-01-0403.

73. Dutta, S. and Laha, M.K.: “Analysis of the small amplitude sloshing of a liquid in a rigid container of arbitrary shape using a low-order boundary element method”, Int. J. Numer. Meth. Engng., 47:1633-1648, 2000.

74. Kang, X, Rakheja, S. and Stiharu, I. :’Directional Dynamics of a Partly-Filled Tank Vehicle under Braking and Steering”, SAE paper 2000-01-3477

75. Jean Ma and Mohammad Usman: "Modeling of Fuel Sloshing Phenomena Considering SolidFluid Interaction", 8th International LS-DYNA User Conference 
76. S. Srikantan, S. Yerrapalli, H. Keshtkar, "Durability Design Process for Truck Body Structures”, Int. J. of Vehicle Design, vol. 23, Nos. 1/2, pp.94-108, 2000.

77. J. Dakin, P. Heyes, M. Fermaer, D. Minen, “Analytical Methods for Durability in the Automotive Industry”, SAE Paper 2001-01-4075.

78. MSC Nastran Advanced Dynamic Analysis user’s Guide, v.70.

79. R. D. Cook, D. S. Malkus, and M. E. Plesha, “Concepts and Applications of Finite Element analysis”, John Wiley \& Sons, Inc., 1989.

80. MSC Fatigue User’s Guise, 2004.

81. nCode, FE-Fatigue User Manual and Theory.

82. T. Rose, "Using Residual Vectors in MSC/NASTRAN Dynamic Analysis to Improve Accuracy”, 1991 MSC World Users’ Conference.

83. MSC Nastran Release Guide, 2004.

84. W. J. Anderson, “Numerical Acoustics”, University of Michigan, Automated analysis Corp., 1996.

85. MSC/NASTRAN Quick reference Guide

86. Peyman Aghssa, Miloslav Riesner, Ford Motor Company, Gerard Winkelmuller, Mecalog, Dimitri Nicolopoulos, Igor Antropov, and David Johnson, Radioss Consulting Corporation, "The Use of Finite Element Method in Computing the Dynamic Pressure inside a Fuel Tank to Simulate a Laboratory Test", IRUC'99 - International Radioss Users Conference -June 21 \& 22 - Sophia Antipolis - France

87. José Luís Farinatti Aymone, D.Sc., "Computational Simulation of 3-D Metal Forming Process using Mesh Adaptation", 2002 SAE BRASIL. 
88. Kouji Kamiya, Yoshihisa Yamaguchi, and Edwin de Vries, "Simulation Studies of Sloshing in a Fuel Tank", 2002 SAE

89. RADIOSS CRASH VERSION 4.2 IMPUT MANUAL

90. RADIOSS CRASH M-CRASH TRAINING MAUNAL 


\begin{abstract}
ARBITRARY LAGRANGIAN-EULERIAN METHOD INVESTIGATION ON FUEL TANK STRAP SIMULATION UNDER PROVING GROUND CONDITION
\end{abstract}

by

GUANGTIAN SONG

August 2016

Advisor: Dr. Chin-An Tan

Major: Mechanical Engineering

Degree: Doctor of Philosophy

The Arbitrary Lagrangian-Eulerian (ALE) is a hybrid finite element formulation that can alleviate many of the drawbacks from the traditional Lagrangian-based and Eulerian-based finite element simulations, which is developed through combining modern algorithms for Lagrangian hydrodynamics, meshing technology and remap methods developed for high-resolution Eulerian methods. Lagrangian-based finite element formulations is that the computational system moves with the material and main drawback is that it will face severe problems to deal with strong distortions in the computational domain. Eulerian-based finite element formulations is that the computational system is a prior fixed in space and unable to deal easily with fluids undergo large distortions at the interface. The use of Arbitrary Lagrangian-Eulerian (ALE) computer codes has been an enabling technology for many important applications. When using the ALE technique in engineering simulations, the computational mesh inside the domains can move arbitrarily to optimize the shapes of elements, while the mesh on the boundaries and interfaces of the domains 
can move along with materials to precisely track the boundaries and interfaces of a multi-material system.

In automotive CAE durability analysis, simulation of dynamic stress and fatigue life of fuel tank straps is a complex problem. Typically a fuel tank is held with fuel tank straps. Its movement lies in the domain of nonlinear large rotation dynamics. Moreover, the sloshing behavior in the fuel tank makes the problem even more intricate.

The objective of this study is to investigate the advantage of ALE method in simulating fuel sloshing through fuel tank and fuel tank strap movement under proving ground conditions using the nonlinear large rotation dynamic method with RADIOSS, a commercial code. After the stress distribution of the fuel tank strap is achieved, a commercial fatigue code, nCode DesignLife, is used to predict the fatigue life of the fuel tank straps.

In this research, the stress distribution of the fuel tank strap can be predicted with Arbitrary Lagrangian-Eulerian Method (ALE) to simulate fuel sloshing which plays critical role in fuel mass redistribution and the stress variation with time. A commercial fatigue code, nCode DesignLife, is used to predict the fatigue life of the fuel tank straps. The analyses have accurately predicted the crack initiation location and sequence in the fuel tank straps, and show good correlation with test. The utilization of this method can give design direction to minimize the iteration of lab testing and expedite the design period. 


\title{
AUTOBIOGRAPHICAL STATEMENT
}

\author{
Guangtian Song
}

\section{Education}

- 2008-2016, PH.D., Mechanical Engineering, Wayne State University

- 1996-1999, MS, Aerospace Engineering, University of Cincinnati

- 1989-1993, BS, Mechanical Engineering, Harbin Engineering University

\section{Work Experience}

- 2015-current, Ford Motor Company, Advanced Chassis Architecture System Engineer

- 2007-2015, AM General, Chassis Engineering Testing IPT Lead and CAE Integration Responsible

- 2005-2007, Daimler Chrysler Corporation, Senior CAE Engineer

- 1999-2005, Ford Motor Company, CAE Engineer

\section{Selected List of Awards}

- 2014 SAE Forest R. McFarland Award

- 2007 North America Hypermesh Technology Conference Excellence Award.

\section{Selected List of Publications}

- Song, G. and Tan, C., "Door Slam CAE Method Investigation," SAE Technical Paper 2015-011324, 2015, doi:10.4271/2015-01-1324.

- Song, G. and Tan, C., "Shell Elements Based Parametric Modeling Method in Frame Robust Design and Optimization," SAE Int. J. Mater. Manuf. 4(1):716-723, 2011, doi:10.4271/2011-010508.

- Song, G. and Tan, C., "Vehicle and Occupant Safety Protection CAE Simulation," SAE Int. J. Mater. Manuf. 3(1):750-758, 2010, doi:10.4271/2010-01-1319.

- $\quad$ Song, G., Shu, K., Khatib-Shahidi, B., Ourchane, A. et al., "Nonlinear Dynamic Simulation of Fuel Tank Strap Stress and Fatigue Life under Proving Ground Conditions," SAE Technical Paper 2005-01-0979, 2005, doi:10.4271/2005-01-0979

- "Strength Simulation of Woven Fabric Composite Materials With Material Nonlinearity Using Micromechanics Based Model”, Journal of Thermoplastics Composite Materials, 16(1)5-20, Jan. 2003

- "Similarity Conditions for Cylindrical and Flat Sandwich Panels", Proceedings of the 1999 ASME Summer Applied Mechanics and Materials Conference, Eds. R.C. Batra and E.G. Henneke, ASME Press, New York, p. 324.

- "Similarity Conditions for Sandwich Shell-Like Configurations", Proceedings of the 1999 ASME International Congress. Advances in Aerospace Materials and Structures, Ed. G. Newaz, ASME (AD-Vol. 58), New York, pp. 65-78, 1999 